\title{
International Petroleum Statistics Report
}

August 1998
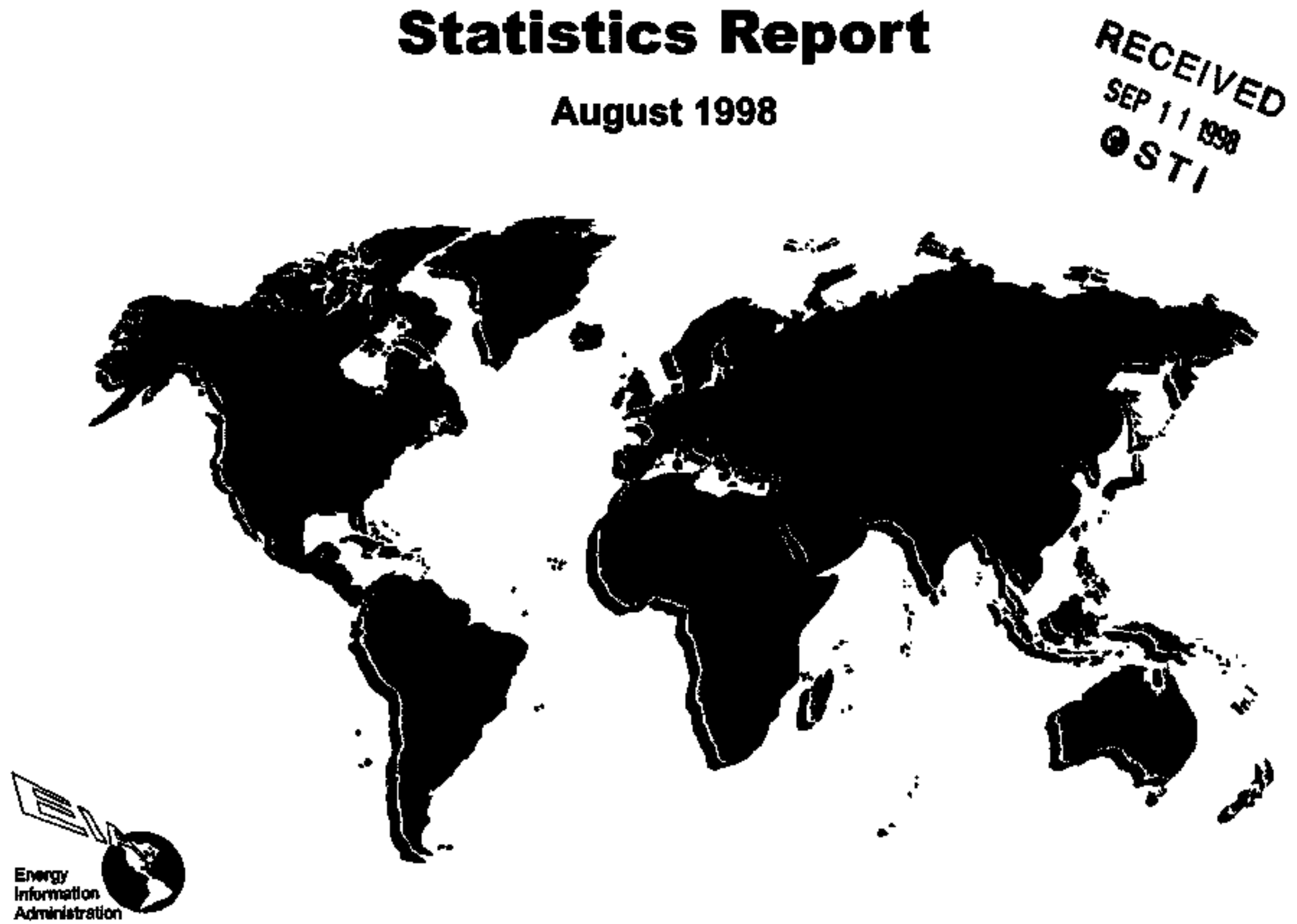


\section{HOW TO OBTAIN EIA PRODUCTS AND SERVICES}

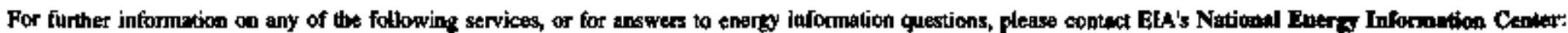
National Eneray Iaformation Center (NEIC)
(202) $586-8800$
Enerty Information Adtrinistration
(202) 5860727 (fax)
Forrestai Buikling, Room IF-048
TTY: (202) 586-1181
Washingtom, DC 20585
E-mail: infoctrQciadoe,gov

\section{Eloctrouc Productu asd Sarvious}

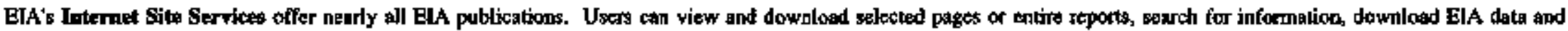
analysis applications, and find out about new ElA infocmation products and services.
World Wide Web: Hrtpat/wrw.etadoengow
Gowher: gophert/sowherdeindoe.gov
FTP: Mpolitp.eindoe.gor

EJA also offers a listserve service for ELA press releases and othex short documents. Sign up on the ELA World Wide Web site.

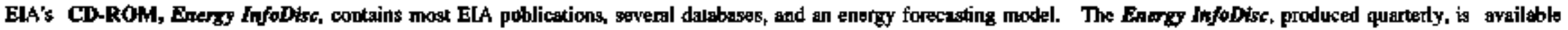
for a fee from STAT-USA, Departmeal of Commetie, I-800-STAT-USA,

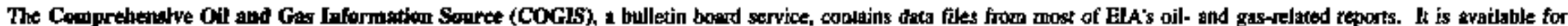
a foe from STAT-USA, on 1-BDO-STAT-USA.

ELA's Electroate Fablishing Syeten (EPUB) bulkelin board comains data files, firoctories, and forecasts from most ElA reports. It can be accessed iree of charge by dialing (202) 586-2557.

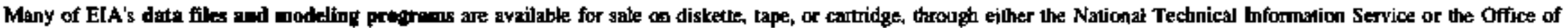
Scientific and Technical Information, Department of Energy. Contact NEIC for infocmation on specific producto, scrurces, and medla, and ordering instructions.

\section{Prinded Publicutions}

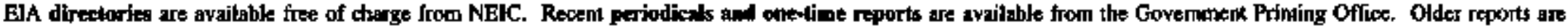
available from the Nalkonal Technical teformution Service:
Superiatendent of Docmunentis
Nationd Techndcal Intornanien Service
U.S. Oovermment Printing Office
U.S. Departinent of Commerce
P.O. Bax 371954
Pittsborgh, PA 15250-7954
Springfield, VA 22161
(202) 512-1800; (202) $512-2250$ (fax)
S285 Port Royal Row
(703) 487-4650; (703) 321-854? (fax)

Released for Printing: August 14, 1998

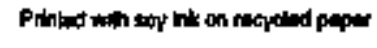

Ouestions conceming the contents of this report should be directed to Michael J. Grilot, (202) 586-6577. 


\title{
International Petroleum Statistics Report
}

\author{
August 1998
}

Eunerory Information Adninktration

Office of Energy Markets and End Use

U.S. Departument of Energy

Waskington, DC 20585

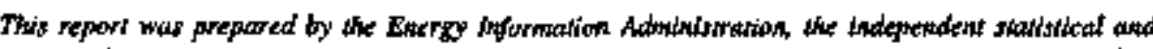

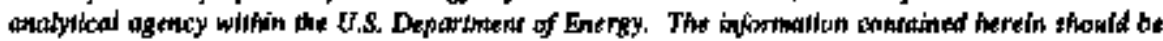

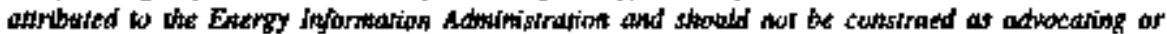

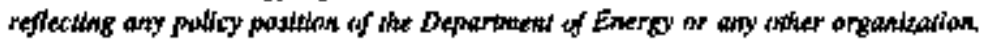




\section{Preface}

The Intemational Petroteum Statistics Report is a monthly publicstion that provides curent intenational oil data. This neport is published for the use of Members of Congress, Federal agencies, State sgencies, indusiry, and the gentral pablic. Publicalion of this report is in keeping wilh responsibilities given the Energy Informalion Adtrinistration in Public Law 95-91 (Section 205(a)(2)) that stales:

"The Administrator shall be responsible for carying out a central, cornprehensive, and unified energy data and information program which will collect, evaluate, assemble, ansalyze and disseminate data and information ..."

The Intemational Petroketam Statistics Repotat presents data on incenational oil prodhction, demand, imports, and stocks. The report has four sections. Section I conkains time series data on work oil production, and on cil demand and stocks in the Organization for Economic Coxperation and Development (OECD). This section contains annul dare begimning in 1985, and monthly dats for the most recest 1wo years. Section 2 preserns ant oil staply/demtand balante for the world. This balance is presented in quarterty intervals for the most recem two years. Section 3 presents deta on ofl imports by OECD couneries. This section contains ammal dala for the most rexent year, quarterly data for the most recent two quanters, and monthly data for the most recent twelve months. Section 4 presents antial time series dava on woeld oil production and oil stocks, demand, and trate in OECD coumtries. Word oil production and OECD deanand data are for the years 1970 through 1997; OECD sioks from 1973 through 1997; and OECD trade from 1987 through 1997.

Data for the Unitcd States are devcloped by the Energy Information Administration's (EIA) Office of Oll and Gas. Data for other costuties are derived langely from published

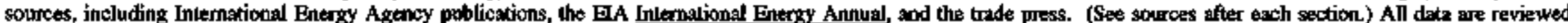
by the International Enetgy Statisties Tean of EJA. All data have been contiverted to units of measuremenl familiar to the American public. Definitions of oil production mod consumption are consistem with other ElA publications.

General infomation about this publication may be oftrined from W. Calvin Kilgore, Dinstor, Office of Energy Markets and End Use, (202) 586-1617, and from Louis D. DeMouy, Director, Integrated Energy Statistics Division, (202) 586-6557. Dexailed questions and comitents concening the contents of the Intemational Putroleum Stutisics Report may be dirocted to Michael J. Grillot, Team Loader, International Energy Stalistics Team, (202) 586-6577, Patricia A Srnith (202) 586-6925, or H. Vicky McLaine (202) 586-9412; Oil Market Chrooology - Douglas Macintyre (202) 586-1831. 


\section{DISCLAIMIER}

This report wh perpared as an ccount of wort sponsoned by an erency of the United States Gowernment. Netilher the United Stules Goverament nor an agency thereof, not any of their employest, maires any werranty, expitas or implied, of

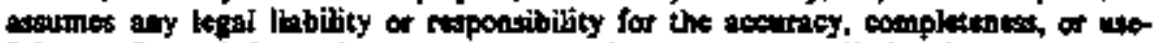
fulnets of sily informtion, apperntus, peoduce, of process disclosed, of represents that ís upe woald not infinge privatefy owned rights. Refarence herein to any spe-

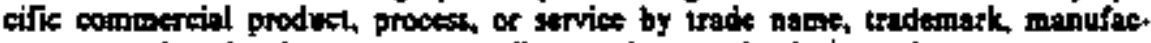
turer, or aherwise does nol necessarily constitule or jmply is endorsement, resom.

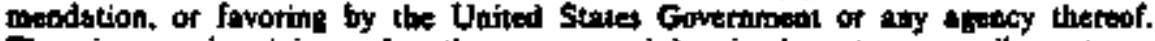

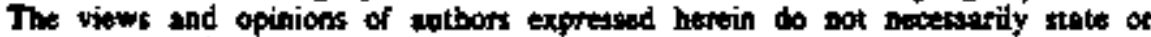

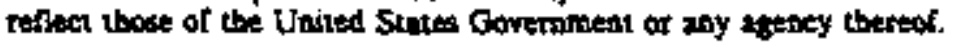




\section{DISCLAIMER}

Portions of this document may be illegible electronic image products. Images are produced from the best available original document. 


\section{ON Martiet Chrowology -- July $19 \%$}

The following chronelogy lists international events of potential significance for world petroleum mankels. Sources include: Dont Jones (DJh, Los Angeles Tinnes (LAT), The New Yonk Times (NYT), The Washington Past (WP), and The Wall Street Joumal (WSt).

Juby 2 Iran's Nariontl Iranian Oil Company (NłOC) announces that it is seetring foreign investors for a number of onshore and offshore developrent projects. The onshore projects inchude the Ceshmeh Khosh gas bjjection project and the Darqualm, Masjed-e-Sukeyman, Agha Jard, and the Central Zagpog oll fiekts, all of which would be offered on a buyback basis. Offshore development projocts inclede the Nowrooe, Foroozan, Salaman, Sìj C and D blocks, Hendajan, Soroosh, Sounth Pars oil fields, and a pilot developmenl a Esfaediar. NIOC says that foreign investments may help fran íncrease its offshore oil production capacity by 276,000 baurels per day by 2000 from a current sate of approximately 500,000 barmels per day. (DJ)

Amoco Kazalhstan and Tukish Petroleum Ovesseas form st equally-held joint venlure, Tepco LLC, to explore for and produce oil and natural gas onstione in western Kazaktistan. Under the terms of the production sharing agreement, Tepco will commence inamediate exploration of Central Territory Bkack B. Turkish Petrolemm is a unit of Tunt Petolleri Anonim Ontakligi, Turkey's stane cil compary. (DJ)

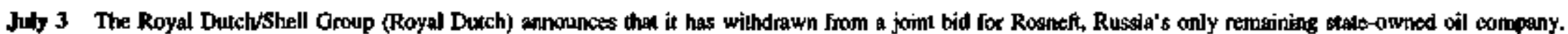
Royal Dutch says that low oil prices. which would fimail returns frotn the project, and friancial temmoil in Russia contributed to its decision to pull out of the bid. Royal Detch's Russian partners in the bid are Lakoil and Gazprom. (NYT)

Jwy 5 Iraq and Jordan sign san agreement for the construction of an oil pipeline between the two coumtries. The pipeline is expected to replace a fleet of moje than 3,000

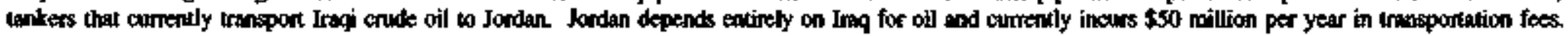
(D)

Juty 6 Russie and Kazakhstan sign en agreement thas tivides the aonthern part of the Caspian Sta seabed into separate Russian and Kazakh sectors, recognizing Kazakbstan's claim to the oil near its coestline. The agreeneat also provides both netions with equal acoess to the Caspian's fishing grounds. The five nations that sumtound the Caspian Sea - Russia, Kazakissan, Aperbajism, Turkmenistan, and lian - have argaed for years ower who owns the oil in the Caspian seabed.

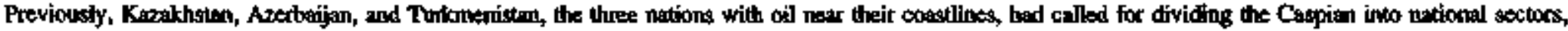

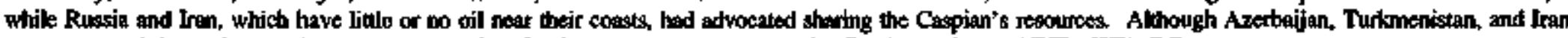
are not part of the seatement, it may sel s precedent for future agreernents among the Caspian nations. (NYT) (WP) (D)

Juty 7 Following the Royal Dutch/Shelt Group's action on July 3, 1998, Bitish Petroleurn (BP) becomes the secood major oft cornpany to withditaw from a possible bid for Rosneft, Russia"s last state-owied oil company. BP imended to bid with is Russian partiner Omeximbank, which controls Russian cil company Sidanco. The

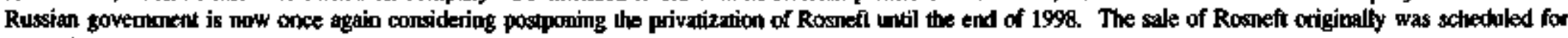
May 1998. (WSJ)

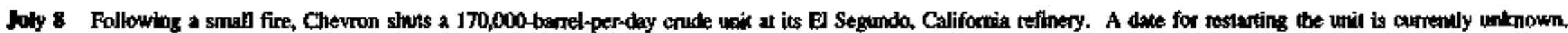
The El Segundo refinery has a tocil crude oil cupacity rating of $\mathbf{2 5 8 , 0 0 0}$ barnels per day. (DI)

Foly 9 For the second time in two months, the Russian gowemmeat postpones the sale of state oil company, Rosneft. The anoomcentent connes just days after British Petnoleum and the Royal Dutchshelt Group wittdrew from their respective bidding partnershipe. Russian officials now say that the sale will talse phace in September or Octoher 1998. (NYT) 
Jaly 9 Mobil of Venezuele announces that Venezutlan enginesing company Vepica and Jantesa and lapan's MCC have won a $\$ 500$ million contract lo develop a crude oil upgrating unit for the Cero Negro heavy cuude project localed in the Orinoco Belt. Mobil is the operatur of the project. The upgrading unit will transform extra-

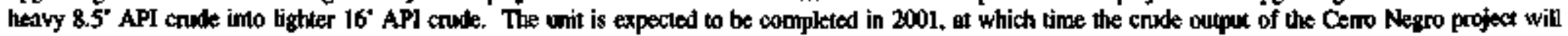
double to 120,000 barrels per day from an inisial level of 60,000 barrels per day in 1999. (DJ)

In its June 1998 monthly oll market report, the Imtemational Evergy Agency (IEA) projects that worht oll denrand in 1998 will be 1.1 miltion bancels per day bigher

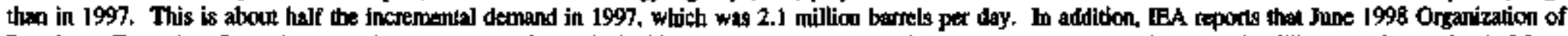

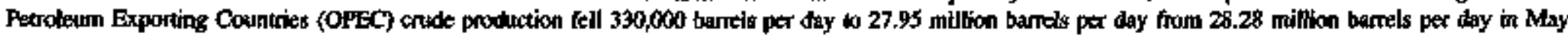
1998. When cominpared to February 1998, OPEC production from non-Iraqi sources hes been corl by 970,000 barrels per day, which is approximately 80 petcent of the 1.245 million-barre]-per-day targe agreed to by OPEC in Mareh A Dow Jones stivey of industry observers reponts similar findings. The survey finds that

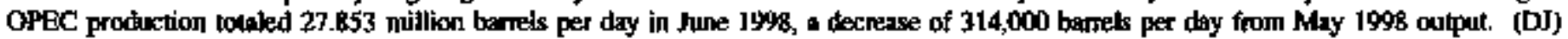

Jnly 10 Monumen Oif and Gas signs a production-sharing agreement with Tukmenneft, Turemenistan's state oil company, and Mobil Exploralion \& Produclion

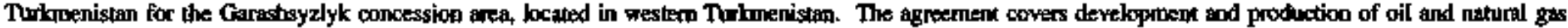

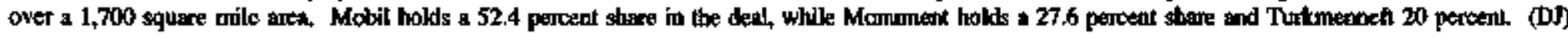

Jaly 13 South Korea's Srmsung agrees to haild a \$240 milbon oil refinery for Ghana's state-owned Tema Oil Refirery. Constnuction of a residtal fhrid catalytic crackiag unit will begin later this year and is expected to be complete in 2001 . The ture will nefine abour 93,000 barrels per day of residual fuel oil, prowucing various types of cil protucts such as gasoline and liquefied peluoleum gas. (DJ)

July 14 Sonangol, Angola's state oil crmpany, and Essor Exploration Angola, a sabsidtiary of Exxom, tiscover a new offshere oil field located abcut 224 miles northrest of Luanda, Angola. The field is called Humgo One and has an estimusted prodkction capscily of 16,000 bantets per day. It is the thitd discovery in Block 15 in tecent tmonths. Essor bolds 240 percent stake in Block 15, with BP Exploration Angola holding 26.67 percent, Italy's Agip Angola 20 percems, wnd Norway's Den Nonske Stats Oljeselskap 13.33 percent. (DJ)

July 15 Iraq and Syria sign an agreement to brild a second oil pipeline between the two countries. No information is available os the new line's capacity or the noute it will take; however, U.S. State Departmetr spokesmen James P. Rutom states thet implementation of the agreement would be a violation of Unitied Nalions sametions mgainst lrag. Two days eartiex, Syria agreed to reopen an existing ofl pipeline linking Jnaq's Kirkuk oil belds to the Mediterranean terminals of Banias in Sycia and Tripoti in Lebanom. Syria closed the line in 1982 in suppon of lnin during the Iraniraq war. The pipetine has an estibrated capacity of 650,000 barreks per dby and is expected to be operaticalal within a few months. However, use of the pipeline will require United Nations approval. (DJ)

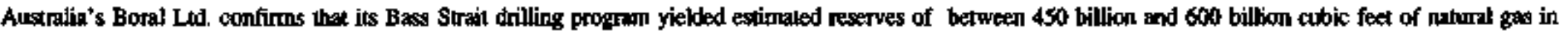
the Yolta Field. Partners in the Yolla Field include Berd with $x$ 30.5 pencent interest, Premier Petrolearm wiah 30.5 percent, Cue Energy Resources with 14 percent,

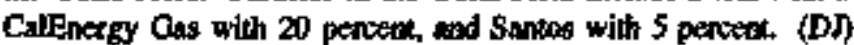

July 16 The U.S. blocks the proposed Angust price fortula foe Iragi crude oil over concens that the Kitkuk grade is priced wo low for the Eumopean manket. It is the first time Ireq's crude oil price formula has been questioned since the Unilted Nacions' ofl-for-food program began in 1996. (DJ)

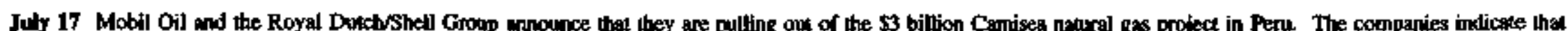

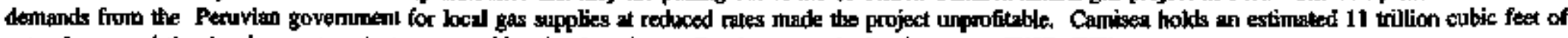
natural gas, and the development project was considered of be a koy to Peru's oconomic development. (WSI) (WP) 
July 29 Abida wibesmen blow up part of Yemen's Marib-Ras lssa oil pipetine in profest of government potce hikes on gasoline, kerosene, and cooking gas. This marks the eighth altack on Yemeni of and gas pipelines since Jume 19, 1998. The 272-mike pipeline carries 220,000 barnels per by of oil and runs from Marib to the Red Sea province of al-Hudaydah, 140 miles west of Yements capital Sanea, (DI)

July 21 Btitish Retroleum, Amoco, and Ramoa Exexgy sigh contracts to develop Azerhaijan's Cosplan Sez oil resources. In thee separate contracts, the companies have pledged to invesl as moch as \$13 billion over the next 15 years. The Caspian Sea region is said to contain npproximately 30 billion tharrels of oil reserves, comparable to the North Sea region. (NYT)

Oil analysts calculats that oil expont revenues of the Organization of Potroteum Exporting Countries (OPEC) have fallen by a third in 1998 due to the decline in oil

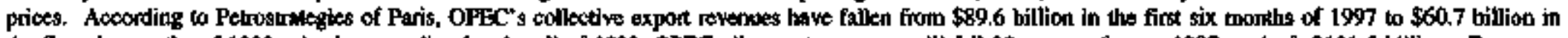
the first six months of 1998 . Analysts pretict that for all of 1998 , OPEC oil export nevenues will fall 30 percent from a 1997 tonat of $\$ 121,5$ billion. Export revenues have declined for almost all OFEC members, with the exception of Irag, whose revenus has increased 20 percent in the first six months of 1998 unter the Unfed Nations' oil-for-fiod progran. (NYT)

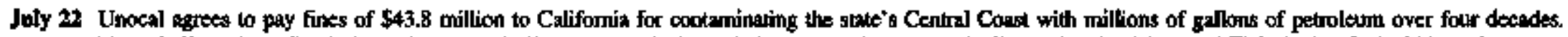
Unocal allowed a nefined vil product, called diluem, wo gradually leak from an underground pipeline at its Guadalupe Oil Field tn San Luis Otispa County. As part of the sertlement, $\$ 9$ million will be used to restone natural resources, $\$ 15$ million will fund water qualiny iroprovement projects, and \$11.1 million will create emergency response programs for future oil spills and other pollution incidents on the Central Coast. (LAT)

In rexponse to pooc market conditions, Singapore Refining Compery (SRC) cuks crude oil throughpul by 45,600 barrels per day, or 16 percent, at its 285,000 -banrelper-day refinery. SRC will continue to nun the refinery at this lower throughput level until mid-August 1998 , when the company will decide on an optimal

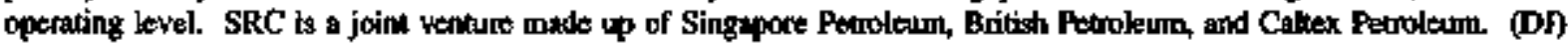

July 23 In response to US. opposition bo the August pricing plan for Iragi crude oil, Iraq offers the United Nations a revised pricints mecharison for Irag's August of

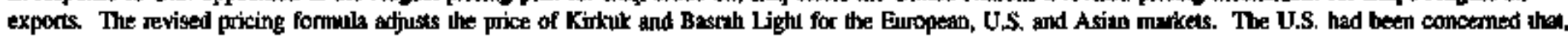
under the oxiginal pricing plan, prices tor crudo oll headed for Europesen mattels were loo low. (DJ)

JHy 27 Russia announces pluns to sell a five peroent stake in RAO Oxzprom, the country's lacgest company and the worlo's targest natural gas compary, with the Russian

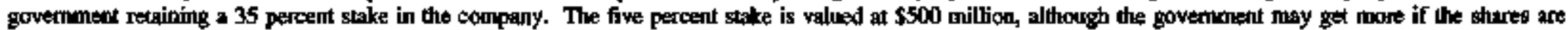

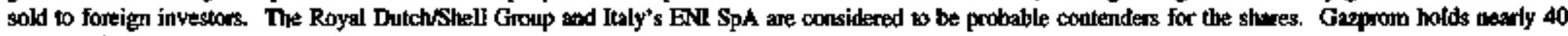
percent of the work's proven nahural ges reserves. (WS)

Nigeria's keader General Abdulsalem Abubakar cancels oil concessions granted to Nigerian companios at the time of previous leader Generd Sani Abechs's death on June B, 1998. General Abubakar also reportedly is no kenger awrending new oil coscessions or peospocting keases. Companies that are affected by the decision include Malabor Petroleum, DICS Petrolteum, Feumarc Pearoleum, and Continental Petroleum. (D)

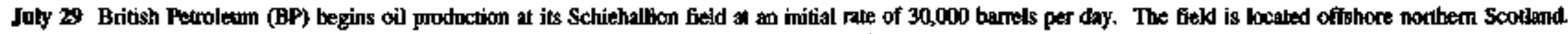
The development is expected to prothce 340 million barrels from the main field and wi addional 85 million barrets from the nearty Loyal reservoir. Peak prefuction is expected to reach 154,000 barmels per day. BP is the operator of the fitld and holds a 33,35 pencent stake. BP's pertiers finchude Royal Durch/Sbell Group (93.35 pencent), Amerade Hess (15.66 percent) and Murphy Oil (5.88 peroent) of the U.S., Norway's Statojl (5.83 percent), and Australia's OMV (5.88 percent). (DI) 


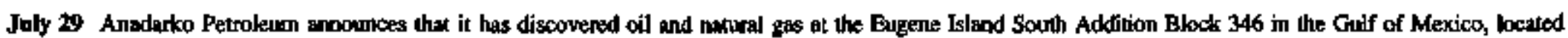

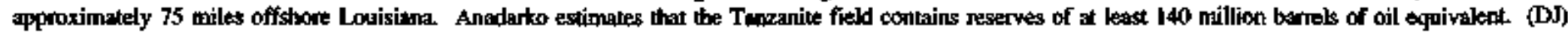

The Indonosian government postpones expkostion of the piax Natuns natural gas field due to the economic crisis in Thailand and the oversupply of liquefied natural gas in the world market. Thailand was expected to be the main buyer of Nahms gas. Production from the field was expected to begin between 2003 and

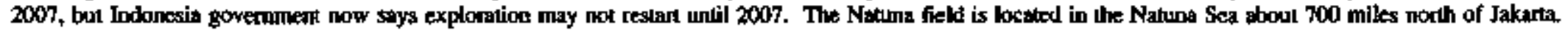
(D)

July 31 The U.S. Senste confinms the nomination of Bill Richardson as Secretary of Energy. Richardson replaces Federico Peina, who resigned in June 1998. (DJ) 


\section{Contents}

\section{Section 1. Anoul arod Monthy OS Duta}

Table 1.1 a Work Crude Oil Prohnction (Including lease Condensale), 1985-Preseat $\ldots \ldots \ldots \ldots \ldots \ldots \ldots \ldots \ldots \ldots \ldots \ldots \ldots \ldots \ldots$

Table 1.1b Wodd Crude Oil Production (Including Lease Condeneate), 1985-Presenx $\ldots \ldots \ldots \ldots \ldots \ldots \ldots \ldots \ldots \ldots \ldots \ldots \ldots \ldots \ldots \ldots, \ldots$

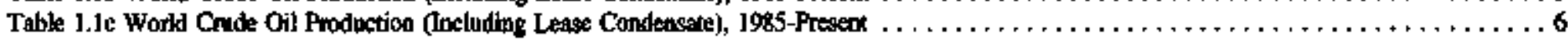

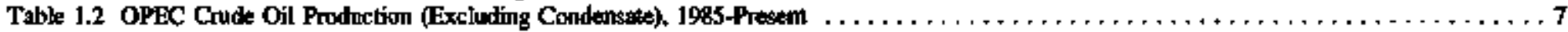

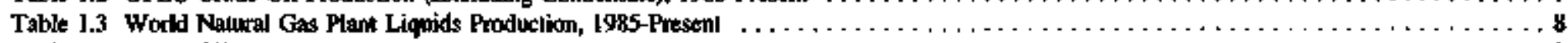

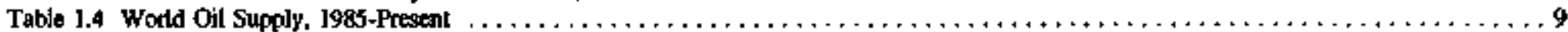

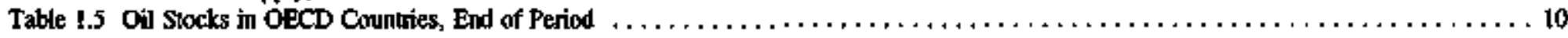

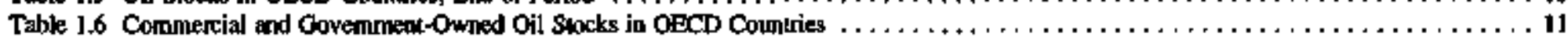

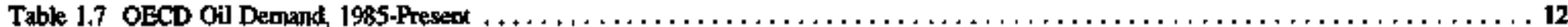

\section{Section 2. Internationel OA Belange}

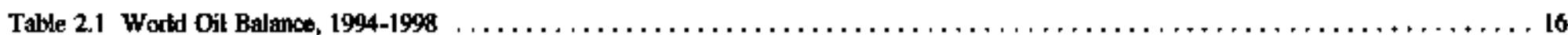

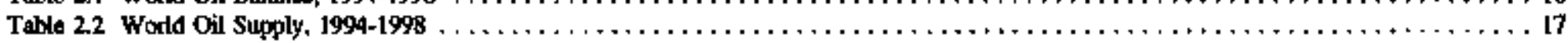

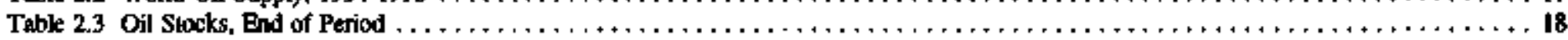

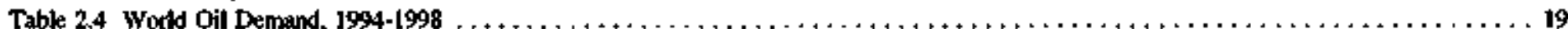

\section{Section 3. Internotional OA Imports}

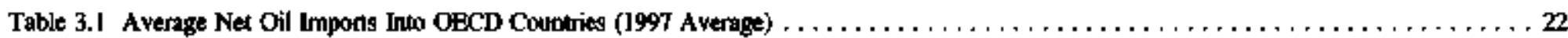

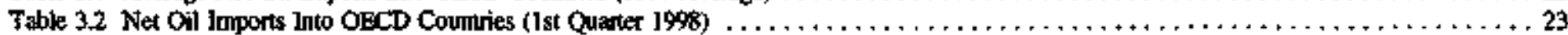

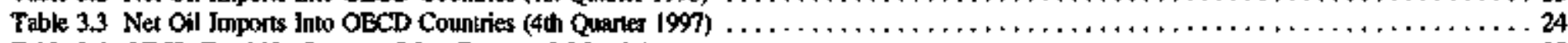

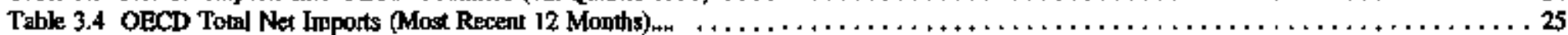

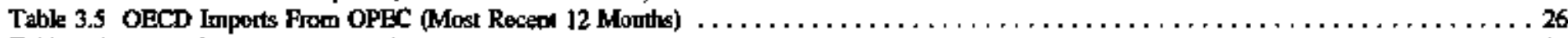

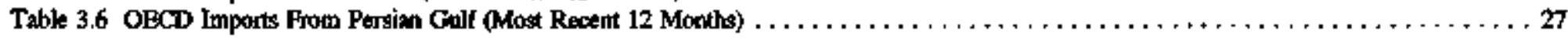

Table 3.7 Uníted States--Net Oil Imporls (Most Recent 12 Months) $\ldots \ldots \ldots \ldots \ldots \ldots \ldots \ldots \ldots \ldots \ldots \ldots \ldots \ldots \ldots \ldots \ldots, 28$

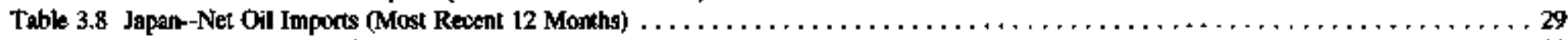

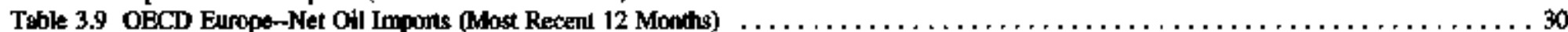

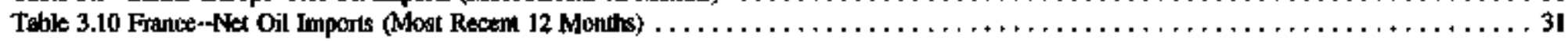

Table 3.11 Genmany-Nat Oil Imports (Most Recent 12 Months) $\ldots \ldots \ldots, \ldots \ldots \ldots \ldots \ldots \ldots \ldots \ldots \ldots \ldots \ldots \ldots \ldots \ldots \ldots \ldots, 32$

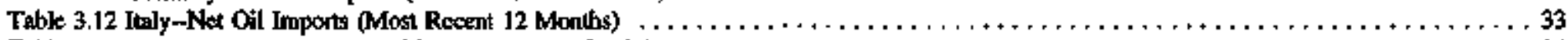

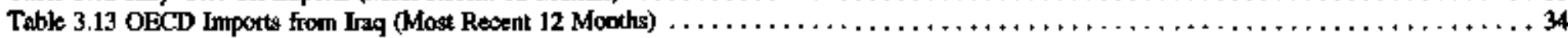

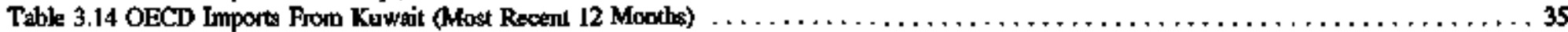




\section{Contents - Continued}

\section{Section 4. Fistarical Oil Dato Series}

Table 4. Ja Wortd Crude Oil Production (Inchuding Lease Condensste), 1970-1997

Table 4.1b World Crude Oil Prodiction (Jincluding Lease Condensate), i970-1997 39

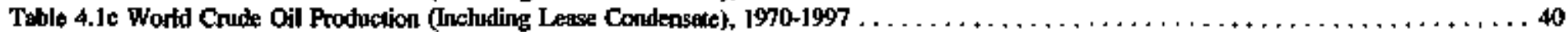

Table 4.2 OPEC Crude Oil Production (Excluding Condenswe), 1980-1997 $\ldots \ldots \ldots \ldots \ldots \ldots \ldots \ldots \ldots \ldots \ldots \ldots \ldots \ldots \ldots \ldots \ldots, \ldots \ldots \ldots$

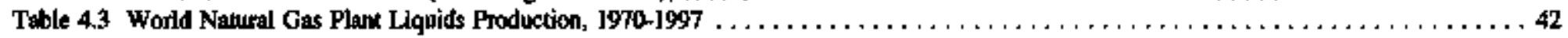

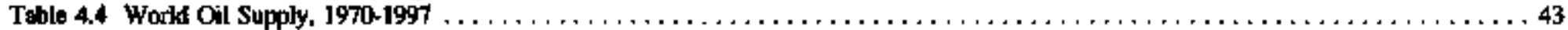

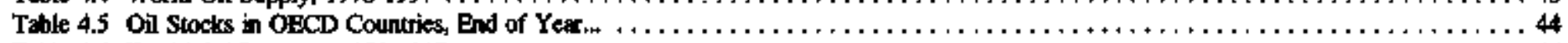

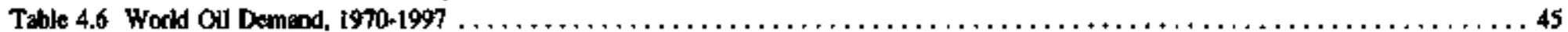

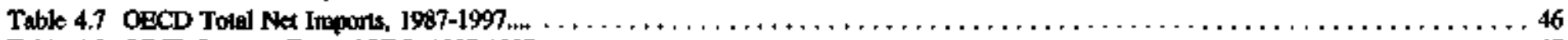

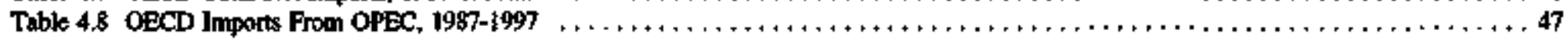

Table 4,9 OECD lmports Frem Persien Golf, 1987-1997 $\ldots \ldots \ldots \ldots \ldots \ldots \ldots \ldots \ldots \ldots \ldots \ldots \ldots \ldots \ldots \ldots \ldots \ldots \ldots \ldots \ldots \ldots$

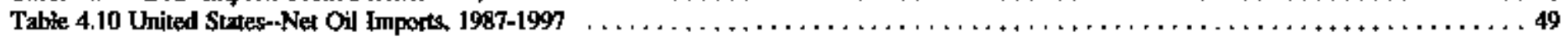

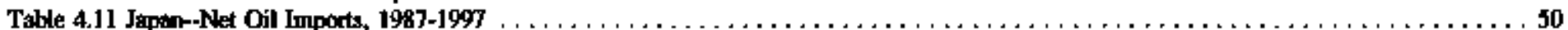

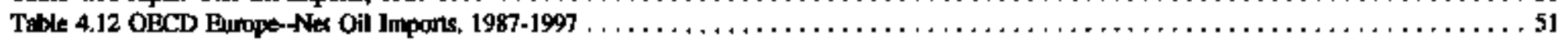

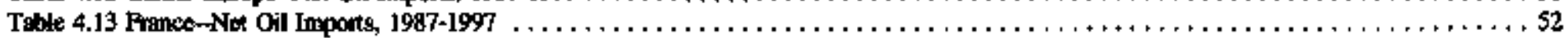

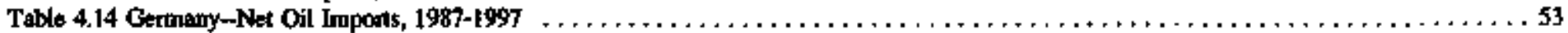

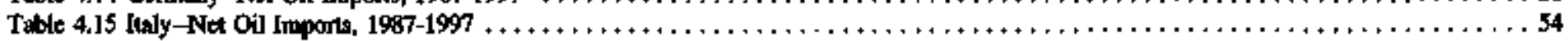

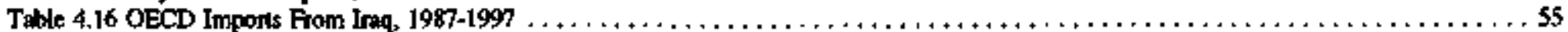

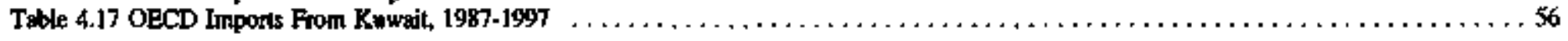

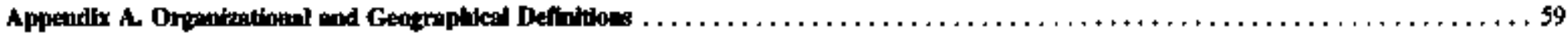

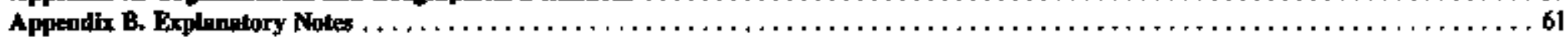

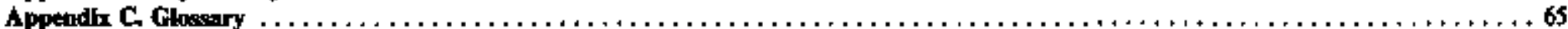

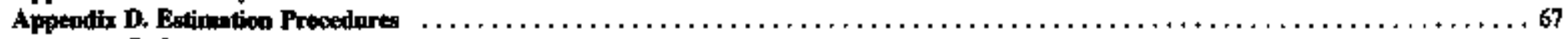

Appartix En Conversions

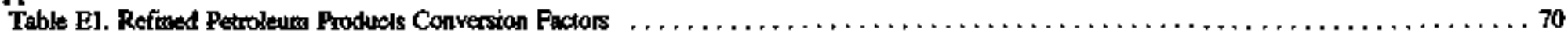

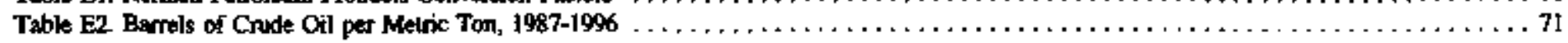

\section{Figures}

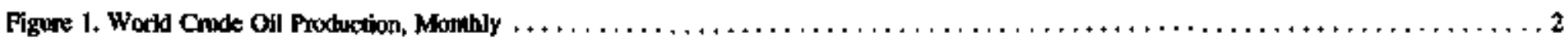

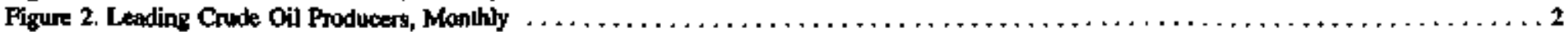

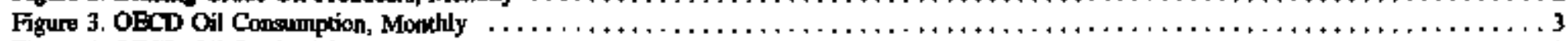

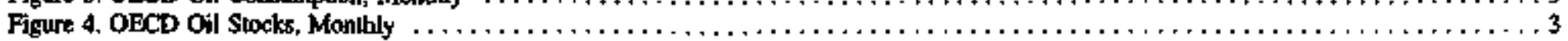


1. Annual and Monthly Oll Data 
F1gure 1. Worla crude oil production, wonthly

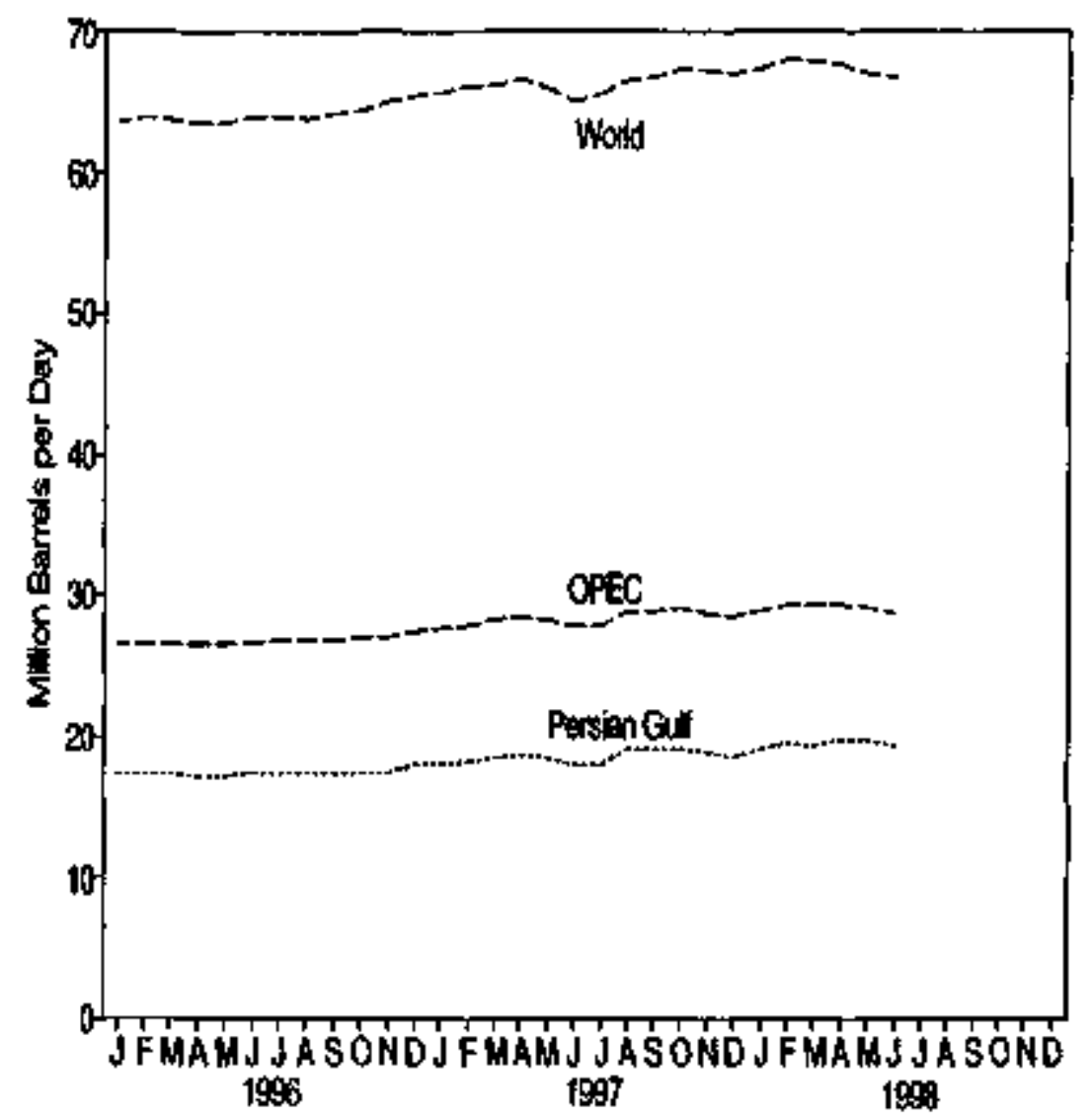

Source: Tabies I.Ia-I, 1c
Flgure 2. Leading Crude 011 producere, wonthly

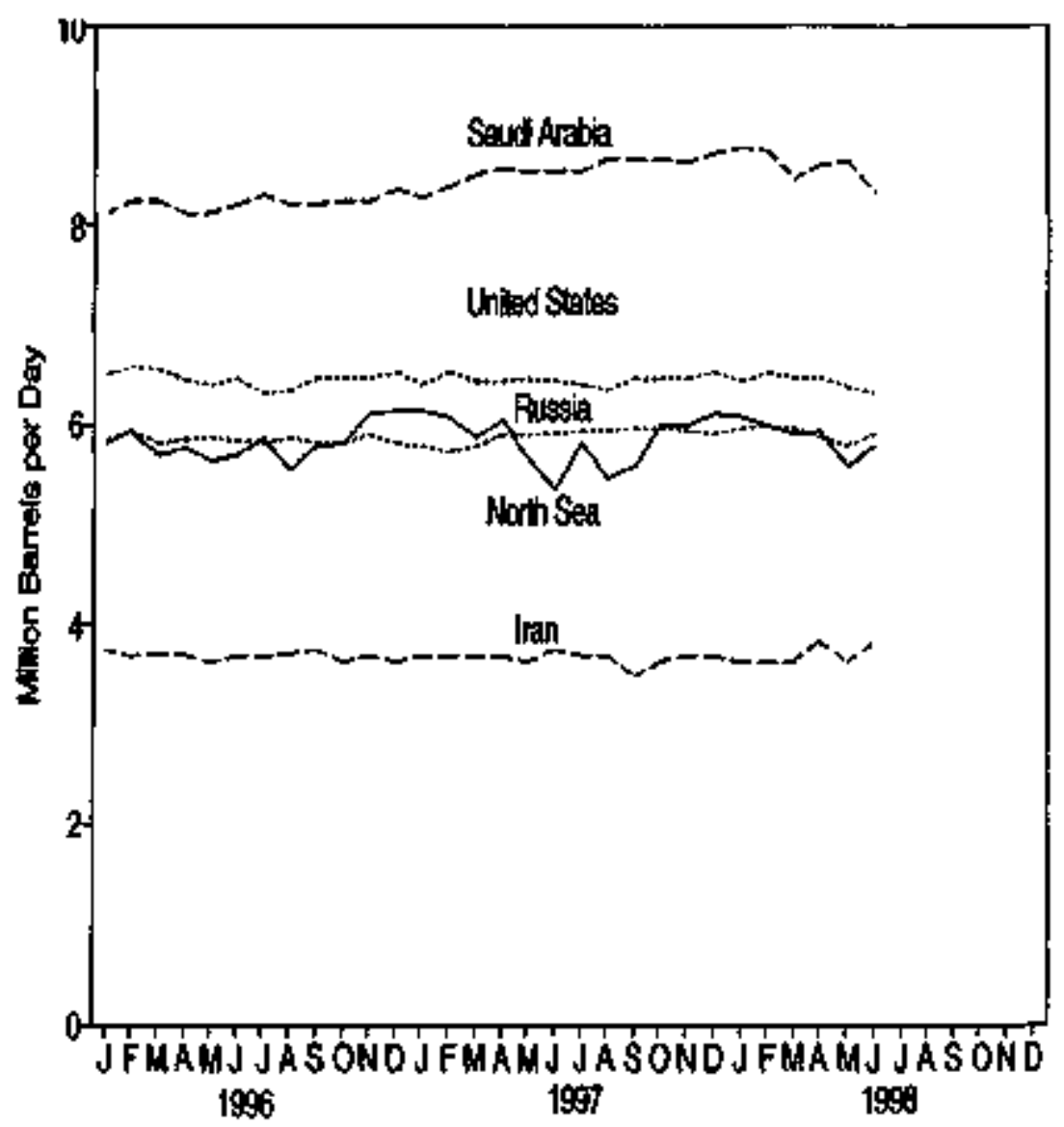

Source: Tablea 1,1a-1,1c 
Flgure 3. OiscD oil Comerimtion, Nonthly

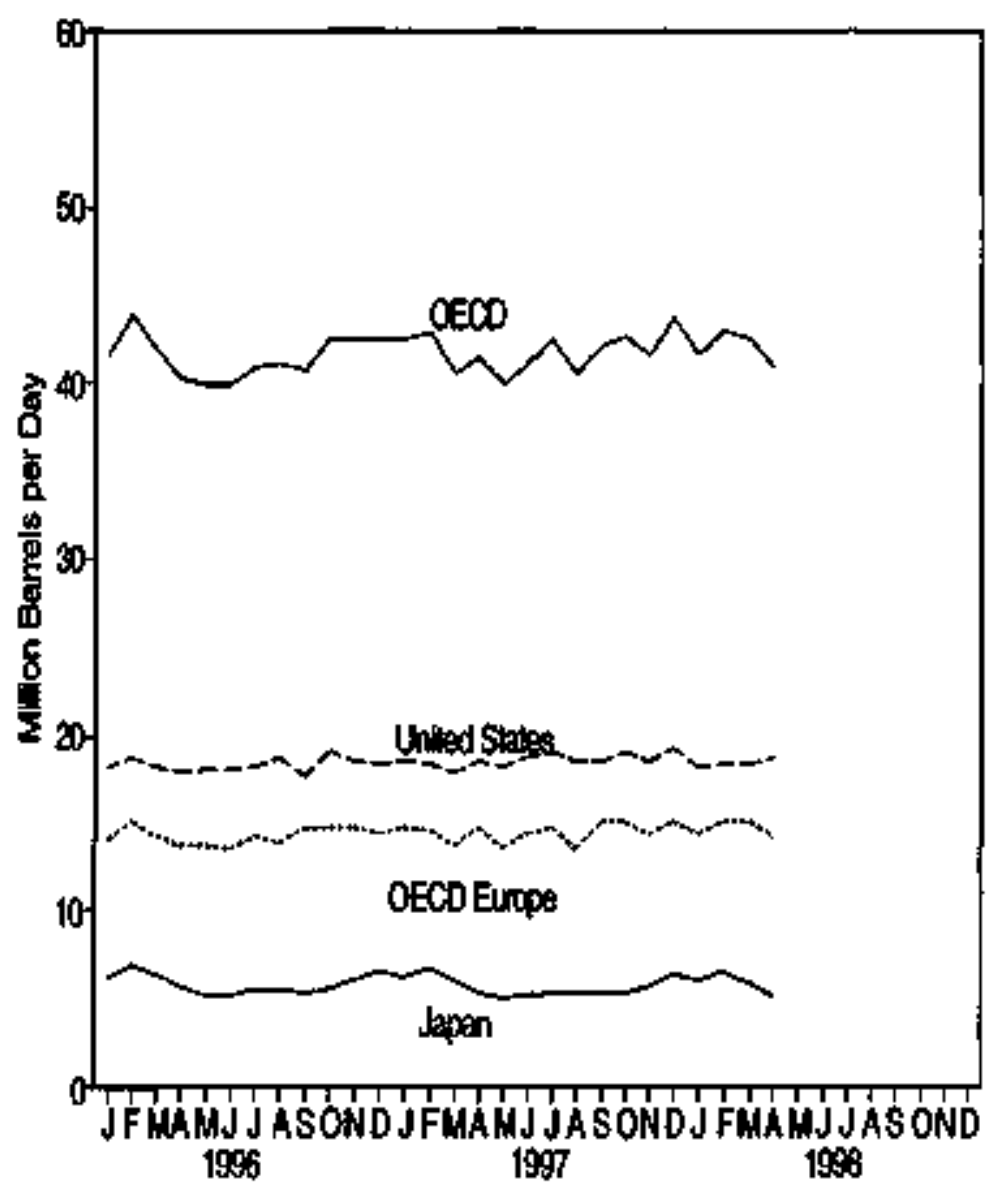

Source: Table 1.7
Figrae 4. Oach oil stooke, lonthly

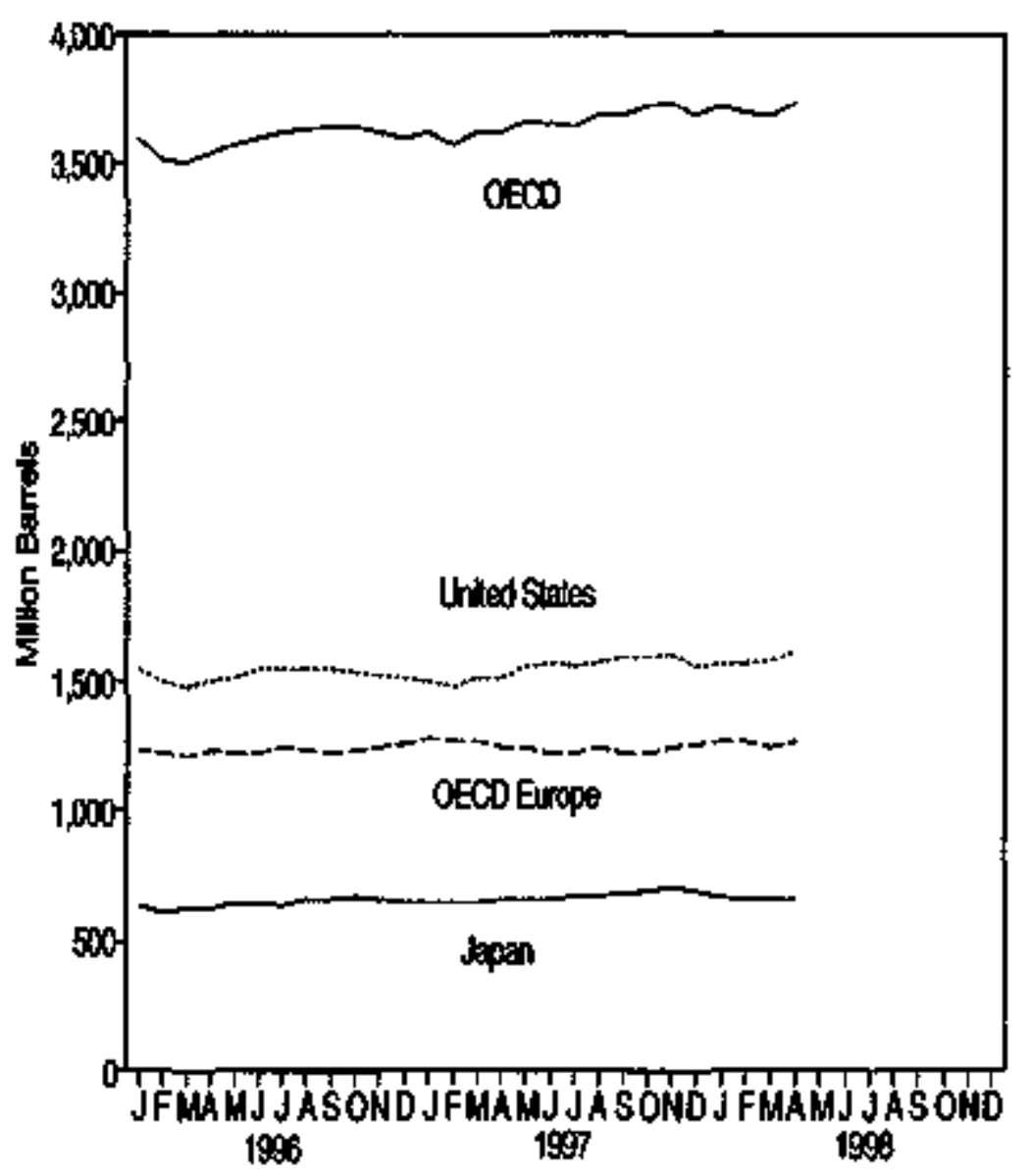

Source: Table 1.5 


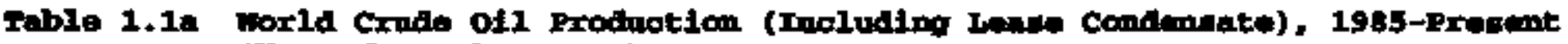
(Thourand barrelle Derr Day)

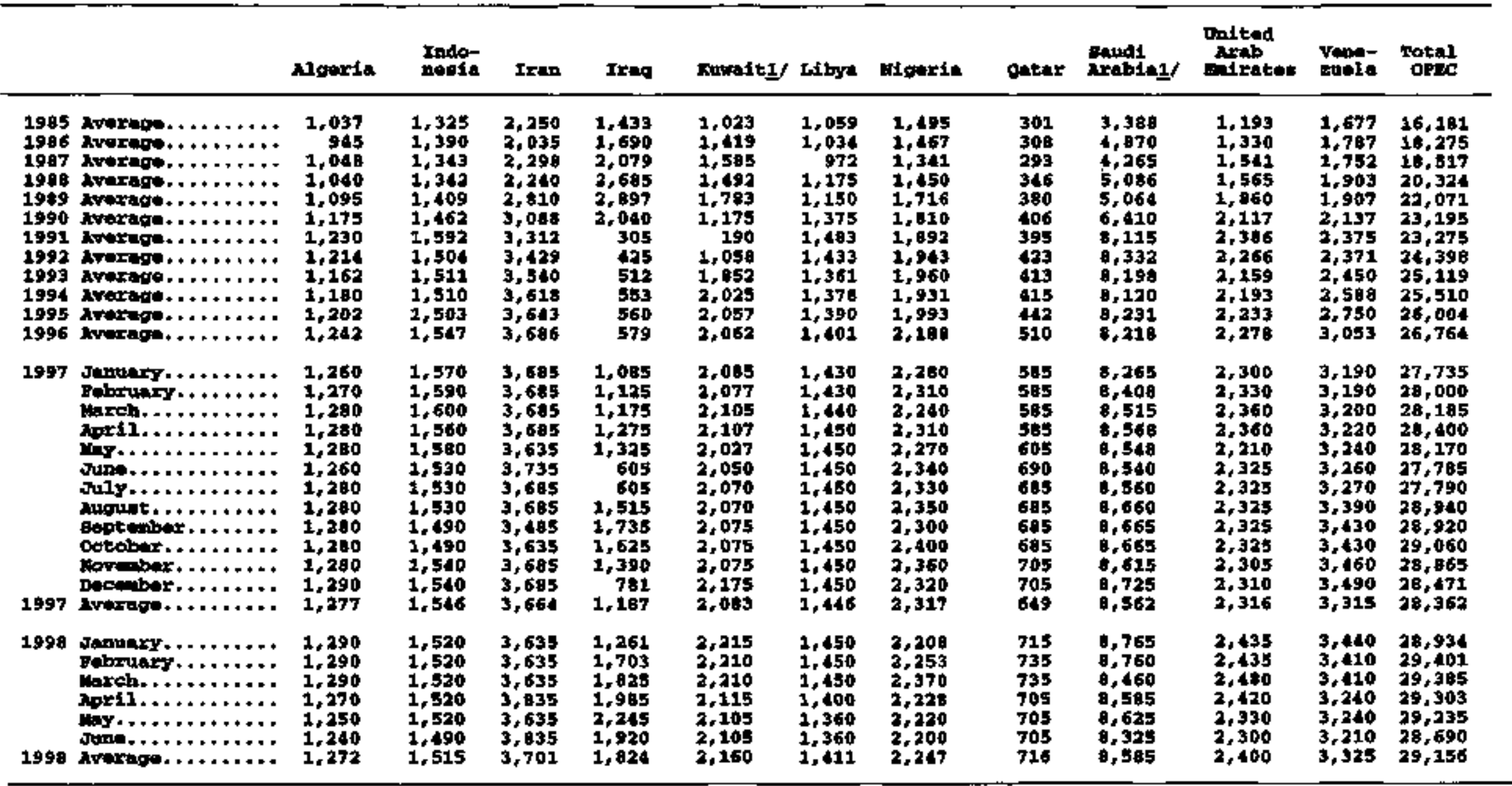

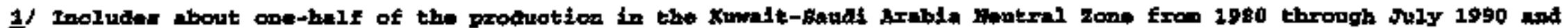

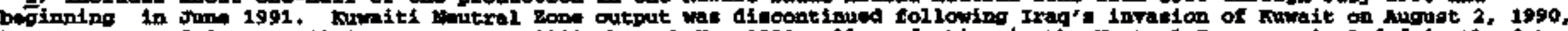

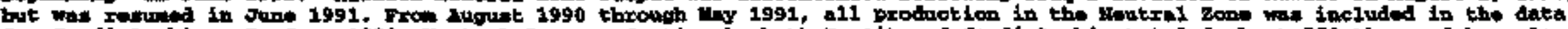

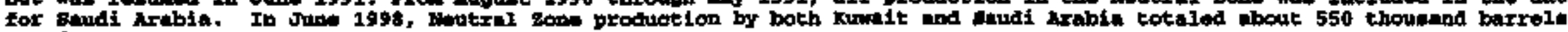
pinz dor.

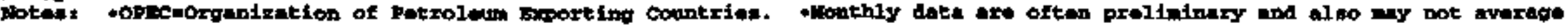

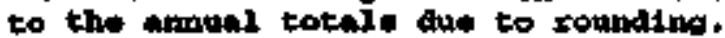

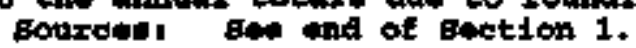




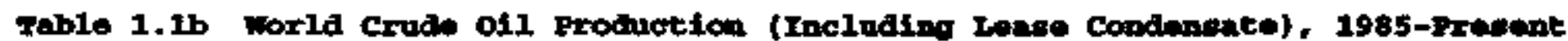
(Fbouland Bariele por Dar)

\begin{tabular}{|c|c|c|c|c|c|c|c|c|c|c|c|c|}
\hline & & Doriving & $\begin{array}{l}\text { Undered } \\
\text { ximydem }\end{array}$ & $\begin{array}{l}\text { Wortb } \\
\text { Toaly }\end{array}$ & nopols & $\begin{array}{c}\text { arotod } \\
\text { tind }\end{array}$ & $\begin{array}{c}\text { hustere- } \\
\text { lig }\end{array}$ & Braz11 & Carnde & Chine & colcubita & Foutudor \\
\hline $\begin{array}{l}1985 \\
1986 \\
1987 \\
1989 \\
1989 \\
1990 \\
1991 \\
1952 \\
1993 \\
1994 \\
1995 \\
1996\end{array}$ & 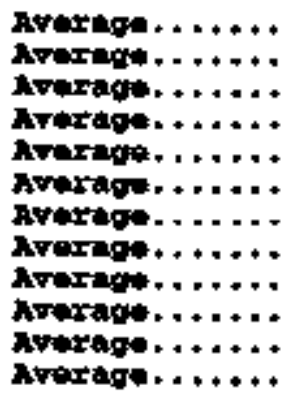 & $\begin{array}{r}788 \\
870 \\
1,022 \\
1,158 \\
1,554 \\
1,704 \\
1,090 \\
2,229 \\
2,350 \\
2,521 \\
2,768 \\
3,104\end{array}$ & $\begin{array}{l}2,530 \\
2,539 \\
2,106 \\
2,232 \\
1,802 \\
1,020 \\
1,797 \\
1,025 \\
1,915 \\
2,375 \\
2,489 \\
2,568\end{array}$ & $\begin{array}{l}3,417 \\
3,547 \\
3,544 \\
3,540 \\
3,515 \\
3,667 \\
3,011 \\
4,182 \\
4,403 \\
5,058 \\
5,400 \\
5,822\end{array}$ & $\begin{array}{l}231 \\
202 \\
360 \\
452 \\
453 \\
475 \\
509 \\
526 \\
609 \\
536 \\
646 \\
709\end{array}$ & $\begin{array}{l}460 \\
434 \\
428 \\
449 \\
460 \\
483 \\
485 \\
559 \\
594 \\
650 \\
715 \\
756\end{array}$ & $\begin{array}{l}575 \\
520 \\
547 \\
538 \\
490 \\
575 \\
545 \\
535 \\
503 \\
536 \\
563 \\
570\end{array}$ & $\begin{array}{l}564 \\
572 \\
566 \\
554 \\
596 \\
631 \\
630 \\
626 \\
643 \\
671 \\
695 \\
795\end{array}$ & $\begin{array}{l}1,471 \\
1,474 \\
1,535 \\
1,616 \\
1,560 \\
1,559 \\
1,640 \\
1,605 \\
1,679 \\
1,746 \\
1,605 \\
1,837\end{array}$ & $\begin{array}{l}2,505 \\
2,620 \\
2,690 \\
2,730 \\
2,757 \\
2,774 \\
2,635 \\
2,645 \\
2,690 \\
2,939 \\
2,990 \\
3,131\end{array}$ & $\begin{array}{l}176 \\
305 \\
385 \\
376 \\
403 \\
440 \\
419 \\
433 \\
456 \\
450 \\
585 \\
623\end{array}$ & $\begin{array}{l}281 \\
293 \\
174 \\
302 \\
279 \\
2 \% 5 \\
299 \\
321 \\
344 \\
365 \\
392 \\
396\end{array}$ \\
\hline 1997 & 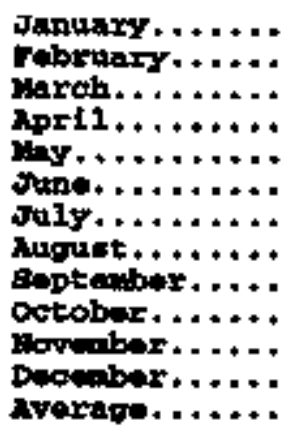 & $\begin{array}{l}3,268 \\
3,263 \\
3,063 \\
3,388 \\
3,194 \\
3,025 \\
3,194 \\
2,890 \\
2,927 \\
3,209 \\
3,192 \\
3,229 \\
3,153\end{array}$ & $\begin{array}{l}2,693 \\
2,660 \\
2,630 \\
2,515 \\
2,315 \\
2,135 \\
2,447 \\
2,407 \\
2,483 \\
2,610 \\
2,602 \\
2,700 \\
2,517\end{array}$ & $\begin{array}{l}6,126 \\
6,074 \\
5,863 \\
6,063 \\
5,677 \\
5,339 \\
5,820 \\
5,462 \\
5,589 \\
6,000 \\
5,992 \\
6,116 \\
5,843\end{array}$ & $\begin{array}{l}700 \\
715 \\
720 \\
720 \\
720 \\
710 \\
715 \\
720 \\
717 \\
725 \\
725 \\
750 \\
720\end{array}$ & $\begin{array}{l}820 \\
820 \\
830 \\
840 \\
840 \\
825 \\
830 \\
640 \\
645 \\
950 \\
850 \\
945 \\
035\end{array}$ & $\begin{array}{l}565 \\
561 \\
594 \\
594 \\
576 \\
574 \\
584 \\
605 \\
626 \\
616 \\
565 \\
590 \\
5 \$ 8\end{array}$ & $\begin{array}{l}840 \\
830 \\
830 \\
830 \\
830 \\
830 \\
860 \\
845 \\
830 \\
740 \\
810 \\
920 \\
933\end{array}$ & $\begin{array}{l}1,874 \\
1,920 \\
1,900 \\
1,823 \\
1,737 \\
1,895 \\
1,089 \\
1,895 \\
1,930 \\
1,956 \\
1,970 \\
1,905 \\
1,893\end{array}$ & $\begin{array}{l}3,210 \\
3,260 \\
3,215 \\
3,230 \\
3,275 \\
3,220 \\
3,190 \\
3,190 \\
3,195 \\
3,195 \\
3,159 \\
3,090 \\
3,200\end{array}$ & $\begin{array}{l}650 \\
640 \\
620 \\
650 \\
650 \\
640 \\
605 \\
635 \\
660 \\
715 \\
745 \\
730 \\
662\end{array}$ & $\begin{array}{l}385 \\
385 \\
380 \\
390 \\
380 \\
360 \\
360 \\
370 \\
390 \\
385 \\
395 \\
390 \\
381\end{array}$ \\
\hline $\begin{array}{l}1998 \\
1998\end{array}$ & 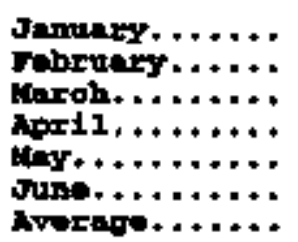 & $\begin{array}{l}3,293 \\
3,230 \\
3,123 \\
3,160 \\
2,917 \\
3,140 \\
3,142\end{array}$ & $\begin{array}{l}2,597 \\
2,583 \\
2,600 \\
2,602 \\
2,499 \\
2,495 \\
2,562\end{array}$ & $\begin{array}{l}6,062 \\
5,989 \\
5,908 \\
5,928 \\
5,589 \\
5,797 \\
5,891\end{array}$ & $\begin{array}{l}735 \\
730 \\
730 \\
710 \\
710 \\
710 \\
721\end{array}$ & $\begin{array}{r}040 \\
040 \\
040 \\
040 \\
2655 \\
055 \\
645\end{array}$ & $\begin{array}{l}528 \\
550 \\
560 \\
604 \\
600 \\
624 \\
570\end{array}$ & $\begin{array}{l}986 \\
920 \\
920 \\
930 \\
930 \\
930 \\
919\end{array}$ & $\begin{array}{r}1,912 \\
1,944 \\
1,952 \\
1,988 \\
1,943 \\
1,932 \\
1,945\end{array}$ & $\begin{array}{l}3,240 \\
3,255 \\
3,170 \\
3,140 \\
3,210 \\
3,260 \\
3,196\end{array}$ & $\begin{array}{r}715 \\
710 \\
725 \\
725 \\
8735 \\
735 \\
724\end{array}$ & $\begin{array}{l}390 \\
380 \\
380 \\
\mathbf{3 8 0} \\
\mathbf{3 1 0 0} \\
380 \\
\mathbf{3 8 2}\end{array}$ \\
\hline
\end{tabular}

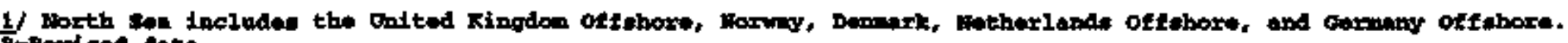
Roreviand atie.

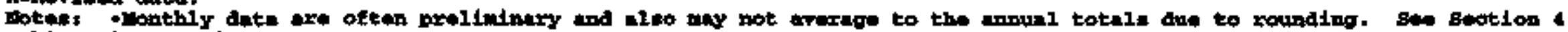
for historical ander.

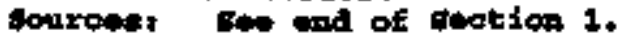




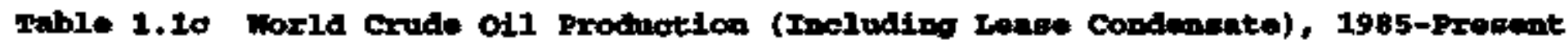
(Whoogand Burrol" per Day)

\begin{tabular}{|c|c|c|c|c|c|c|c|c|c|c|c|c|c|}
\hline & & srpt & Aabon & tadia & Heldy & Nerdoo & Onin & Anosta & $\begin{array}{l}\text { rod } \\
\text { v.s.8. }\end{array}$ & syrie & $\begin{array}{l}\text { Doited } \\
\text { otentel }\end{array}$ & othor $1 /$ & World \\
\hline $\begin{array}{l}1985 \\
1986 \\
1997 \\
1988 \\
1989 \\
1990 \\
1991 \\
1992 \\
1993 \\
1994 \\
1995 \\
1996\end{array}$ & 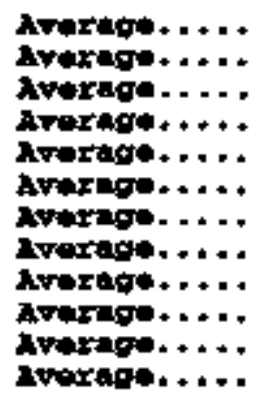 & $\begin{array}{l}067 \\
913 \\
896 \\
948 \\
065 \\
873 \\
974 \\
801 \\
890 \\
996 \\
920 \\
922\end{array}$ & $\begin{array}{l}172 \\
166 \\
155 \\
159 \\
208 \\
270 \\
294 \\
298 \\
313 \\
329 \\
365 \\
368\end{array}$ & $\begin{array}{l}620 \\
630 \\
609 \\
635 \\
700 \\
660 \\
615 \\
561 \\
534 \\
590 \\
703 \\
651\end{array}$ & $\begin{array}{l}440 \\
504 \\
497 \\
540 \\
585 \\
619 \\
646 \\
653 \\
640 \\
645 \\
602 \\
695\end{array}$ & $\begin{array}{l}2,745 \\
2,435 \\
2,548 \\
2,513 \\
2,520 \\
2,553 \\
2,650 \\
2,659 \\
2,673 \\
2,685 \\
2,610 \\
2,855\end{array}$ & $\begin{array}{l}498 \\
560 \\
582 \\
617 \\
641 \\
685 \\
700 \\
740 \\
776 \\
810 \\
951 \\
883\end{array}$ & $\begin{array}{r}= \\
=- \\
=- \\
=- \\
=- \\
=- \\
7,632 \\
6,730 \\
6,195 \\
5,995 \\
5,650\end{array}$ & $\begin{array}{r}11,585 \\
11,995 \\
12,050 \\
12,053 \\
11,715 \\
10,925 \\
9,992 \\
=- \\
=- \\
=- \\
=-\end{array}$ & $\begin{array}{l}170 \\
194 \\
230 \\
265 \\
340 \\
380 \\
492 \\
481 \\
554 \\
560 \\
573 \\
604\end{array}$ & $\begin{array}{l}0,971 \\
6,680 \\
8,349 \\
8,140 \\
7,613 \\
7,955 \\
7,417 \\
7,172 \\
6,847 \\
6,662 \\
6,560 \\
6,465\end{array}$ & $\begin{array}{l}2,124 \\
2,166 \\
2,124 \\
2,235 \\
2,249 \\
2,253 \\
2,274 \\
3,228 \\
3,280 \\
9,982 \\
3,410 \\
3,509\end{array}$ & $\begin{array}{l}53,982 \\
56,227 \\
56,666 \\
50,737 \\
59,863 \\
60,566 \\
60,207 \\
60,212 \\
60,238 \\
60,992 \\
62,331 \\
61,054\end{array}$ \\
\hline 1997 & 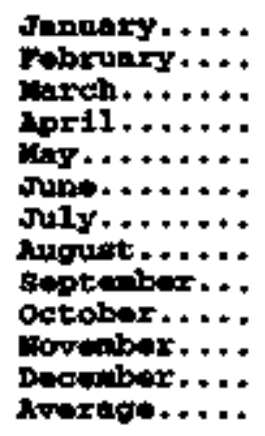 & $\begin{array}{l}685 \\
685 \\
890 \\
890 \\
680 \\
970 \\
880 \\
670 \\
660 \\
660 \\
960 \\
860 \\
874\end{array}$ & $\begin{array}{l}370 \\
370 \\
370 \\
370 \\
370 \\
370 \\
370 \\
370 \\
360 \\
970 \\
370 \\
360 \\
360\end{array}$ & $\begin{array}{l}650 \\
650 \\
660 \\
685 \\
685 \\
685 \\
685 \\
685 \\
675 \\
678 \\
691 \\
675 \\
678\end{array}$ & $\begin{array}{l}730 \\
730 \\
730 \\
750 \\
750 \\
740 \\
740 \\
740 \\
760 \\
760 \\
760 \\
760 \\
746\end{array}$ & $\begin{array}{l}2,940 \\
2,970 \\
2,970 \\
3,945 \\
3,990 \\
3,005 \\
3,035 \\
3,080 \\
3,905 \\
3,087 \\
3,005 \\
3,056 \\
3,023\end{array}$ & $\begin{array}{l}990 \\
900 \\
900 \\
900 \\
990 \\
900 \\
940 \\
970 \\
670 \\
980 \\
990 \\
910 \\
907\end{array}$ & $\begin{array}{l}5,789 \\
5,729 \\
5,772 \\
5,893 \\
5,902 \\
5,902 \\
5,923 \\
5,945 \\
5,958 \\
5,951 \\
5,945 \\
5,893 \\
5,694\end{array}$ & $\begin{array}{l}= \\
=- \\
=- \\
= \\
=- \\
=- \\
= \\
= \\
= \\
=\end{array}$ & $\begin{array}{l}\mathbf{5 6 5} \\
\mathbf{5 6 5} \\
\mathbf{5 6 5} \\
\mathbf{5 6 0} \\
\mathbf{5 6 0} \\
\mathbf{5 6 0} \\
\mathbf{5 6 0} \\
\mathbf{5 6 0} \\
\mathbf{5 6 0} \\
\mathbf{5 6 0} \\
\mathbf{5 6 0} \\
\mathbf{5 6 0} \\
\mathbf{5 6 1}\end{array}$ & $\begin{array}{l}6,402 \\
6,514 \\
6,452 \\
6,441 \\
6,474 \\
6,442 \\
6,409 \\
6,347 \\
6,486 \\
6,467 \\
6,459 \\
6,531 \\
6,482\end{array}$ & $\begin{array}{l}3,715 \\
3,694 \\
3,734 \\
3,756 \\
3,720 \\
3,725 \\
3,679 \\
3,669 \\
3,670 \\
3,684 \\
3,720 \\
3,703 \\
3,706\end{array}$ & $\begin{array}{l}65,676 \\
66,041 \\
66,018 \\
66,571 \\
65,908 \\
65,128 \\
65,576 \\
66,474 \\
66,827 \\
67,361 \\
67,207 \\
67,007 \\
66,317\end{array}$ \\
\hline 1998 & 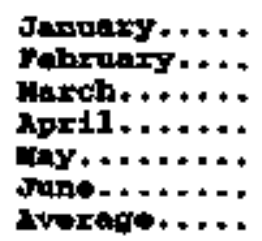 & $\begin{array}{r}860 \\
860 \\
860 \\
860 \\
2870 \\
870 \\
869\end{array}$ & $\begin{array}{l}360 \\
370 \\
370 \\
370 \\
370 \\
370 \\
360\end{array}$ & $\begin{array}{r}680 \\
685 \\
685 \\
635 \\
6770 \\
685 \\
673\end{array}$ & $\begin{array}{r}740 \\
730 \\
740 \\
740 \\
7750 \\
750 \\
742\end{array}$ & $\begin{array}{l}3,085 \\
3,140 \\
3,160 \\
3,140 \\
3,149 \\
3,050 \\
3,121\end{array}$ & $\begin{array}{l}920 \\
920 \\
930 \\
920 \\
910 \\
900 \\
917\end{array}$ & $\begin{array}{r}\mathrm{RS}, 979 \\
\mathrm{RS}, 997 \\
\mathrm{RS}, 962 \\
\mathrm{R}, \mathrm{676} \\
\mathrm{R} 5,789 \\
5,919 \\
5,919\end{array}$ & $\begin{array}{l}=- \\
= \\
=- \\
= \\
= \\
=\end{array}$ & $\begin{array}{l}\mathbf{5 5 0} \\
550 \\
550 \\
550 \\
5501 \\
\mathbf{5 5 0} \\
550\end{array}$ & $\begin{array}{l}86,438 \\
26,538 \\
=6,465 \\
26,484 \\
26,384 \\
26,335 \\
26,439\end{array}$ & $\begin{array}{r}\mathrm{A3}, 731 \\
\mathrm{R3}, 713 \\
\mathrm{H3}, 712 \\
\mathrm{R3}, 673 \\
\mathrm{m3}, 628 \\
3,659 \\
3,686\end{array}$ & $\begin{array}{l}\mathrm{R} 67,414 \\
\mathrm{R} 67,945 \\
\mathrm{R67}, 919 \\
\mathrm{R} 67,630 \\
\mathrm{R} 67,084 \\
66,839 \\
67,449\end{array}$ \\
\hline
\end{tabular}

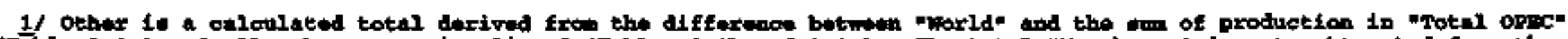

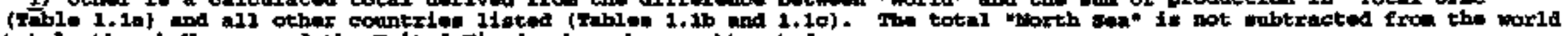

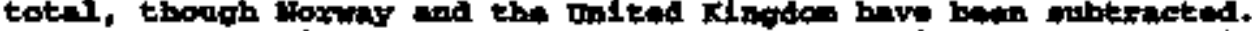

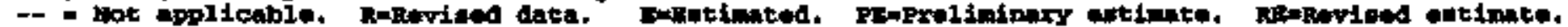

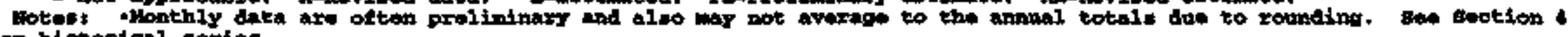
Lox hletorienl gerion.

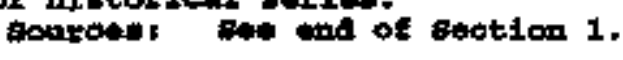




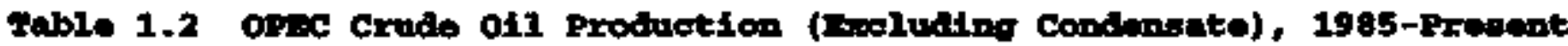

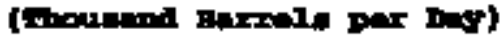

\begin{tabular}{|c|c|c|c|c|c|c|c|c|c|c|c|c|c|}
\hline & & Noerta & Indo- & Iran & Iras & Kutwalte1/ & Libya & Wistrie & Qatax & 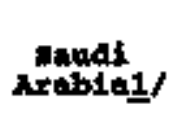 & $\begin{array}{l}\text { Datted } \\
\text { Arab } \\
\text { ditrates: }\end{array}$ & $\begin{array}{l}\text { vero- } \\
\text { zuele }\end{array}$ & Total \\
\hline $\begin{array}{l}1985 \\
1986 \\
1987 \\
1988 \\
1969 \\
1990 \\
1991 \\
1992 \\
1993 \\
1994 \\
1995 \\
1996\end{array}$ & 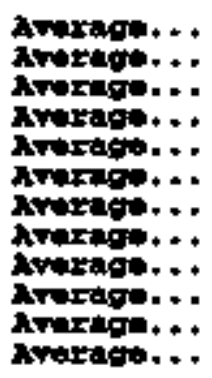 & $\begin{array}{l}702 \\
600 \\
640 \\
640 \\
690 \\
765 \\
900 \\
772 \\
747 \\
750 \\
767 \\
912\end{array}$ & $\begin{array}{l}1,181 \\
1,257 \\
1,189 \\
1,177 \\
1,231 \\
1,281 \\
1,410 \\
1,326 \\
1,327 \\
1,334 \\
1,343 \\
1,367\end{array}$ & $\begin{array}{l}2,250 \\
2,035 \\
2,298 \\
2,200 \\
2,810 \\
3,088 \\
3,312 \\
3,029 \\
3,560 \\
3,600 \\
3,608 \\
3,651\end{array}$ & $\begin{array}{r}1,433 \\
1,690 \\
2,079 \\
2,605 \\
2,897 \\
2,040 \\
305 \\
425 \\
512 \\
548 \\
550 \\
579\end{array}$ & $\begin{array}{l}1,023 \\
1,419 \\
1,585 \\
1,492 \\
1,793 \\
1,175 \\
190 \\
1,058 \\
1,852 \\
0,034 \\
2,087 \\
2,062\end{array}$ & $\begin{array}{l}1,059 \\
1,034 \\
1,979 \\
1,175 \\
1,150 \\
1,375 \\
1,483 \\
1,493 \\
1,361 \\
1,370 \\
1,390 \\
1,401\end{array}$ & $\begin{array}{l}1,475 \\
1,447 \\
1,281 \\
1,400 \\
1,666 \\
1,755 \\
1,832 \\
1,820 \\
1,835 \\
1,897 \\
1,876 \\
2,051\end{array}$ & $\begin{array}{l}301 \\
309 \\
293 \\
346 \\
380 \\
406 \\
395 \\
425 \\
413 \\
409 \\
49 \\
475\end{array}$ & $\begin{array}{l}3,328 \\
4,570 \\
6,263 \\
5,086 \\
5,061 \\
6,410 \\
8,115 \\
8,332 \\
8,198 \\
8,147 \\
8,231 \\
8,218\end{array}$ & $\begin{array}{l}1,133 \\
1,270 \\
1,41 \\
1,465 \\
1,703 \\
2,066 \\
2,326 \\
2,206 \\
2,109 \\
2,193 \\
2,197 \\
2,198\end{array}$ & $\begin{array}{l}1,558 \\
1,645 \\
1,587 \\
1,715 \\
1,747 \\
2,097 \\
2,338 \\
2,334 \\
2,010 \\
2,542 \\
2,710 \\
3,0 \pm 3\end{array}$ & $\begin{array}{l}15,503 \\
17,575 \\
17,640 \\
19,421 \\
21,201 \\
22,559 \\
22,506 \\
23,550 \\
24,304 \\
24,812 \\
23,179 \\
25,926\end{array}$ \\
\hline 1997 & 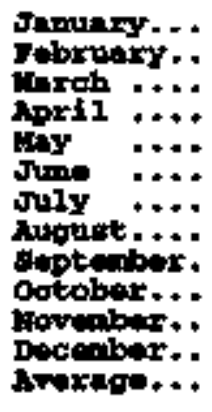 & $\begin{array}{l}930 \\
640 \\
650 \\
650 \\
650 \\
830 \\
690 \\
850 \\
650 \\
650 \\
650 \\
860 \\
647\end{array}$ & $\begin{array}{l}1,390 \\
1,410 \\
1,420 \\
1,390 \\
1,400 \\
1,350 \\
1,350 \\
1,350 \\
1,310 \\
1,310 \\
1,360 \\
1,360 \\
1,366\end{array}$ & $\begin{array}{l}3,650 \\
3,650 \\
3,650 \\
3,650 \\
3,600 \\
3,700 \\
3,650 \\
3,650 \\
3,650 \\
3,600 \\
3,650 \\
3,650 \\
3,629\end{array}$ & $\begin{array}{r}1,080 \\
1,120 \\
1,170 \\
1,270 \\
1,320 \\
600 \\
600 \\
1,510 \\
1,730 \\
1,620 \\
1,385 \\
1,776 \\
1,182\end{array}$ & $\begin{array}{l}2,095 \\
2,077 \\
2,105 \\
2,107 \\
2,027 \\
2,050 \\
2,070 \\
2,070 \\
2,075 \\
2,075 \\
2,075 \\
2,175 \\
2,003\end{array}$ & $\begin{array}{l}1,430 \\
1,430 \\
1,400 \\
1,450 \\
1,450 \\
1,450 \\
1,450 \\
1,450 \\
1,450 \\
1,450 \\
1,450 \\
1,450 \\
1,446\end{array}$ & $\begin{array}{l}2,180 \\
2,210 \\
2,110 \\
2,210 \\
2,170 \\
2,210 \\
2,230 \\
2,250 \\
2,200 \\
2,300 \\
2,260 \\
2,220 \\
2,217\end{array}$ & $\begin{array}{l}550 \\
550 \\
550 \\
550 \\
570 \\
655 \\
650 \\
650 \\
650 \\
650 \\
670 \\
670 \\
614\end{array}$ & $\begin{array}{l}8,265 \\
6,408 \\
0,515 \\
0,569 \\
0,548 \\
0,540 \\
0,560 \\
0,660 \\
0,665 \\
0,665 \\
8,615 \\
0,725 \\
0,562\end{array}$ & $\begin{array}{l}2,220 \\
2,250 \\
2,280 \\
2,280 \\
2,130 \\
2,245 \\
2,245 \\
2,215 \\
2,245 \\
2,245 \\
2,225 \\
2,230 \\
2,236\end{array}$ & $\begin{array}{l}3,150 \\
3,150 \\
3,160 \\
3,180 \\
3,200 \\
3,200 \\
3,230 \\
3,350 \\
3,390 \\
3,390 \\
3,120 \\
3,450 \\
3,275\end{array}$ & $\begin{array}{l}26,930 \\
27,095 \\
27,280 \\
27,485 \\
27,265 \\
26,080 \\
26,005 \\
28,035 \\
26,015 \\
28,155 \\
27,960 \\
27,568 \\
27,457\end{array}$ \\
\hline 1998 & 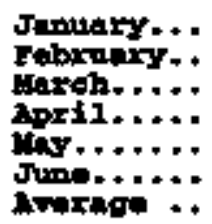 & $\begin{array}{l}860 \\
860 \\
860 \\
840 \\
820 \\
810 \\
842\end{array}$ & $\begin{array}{l}1,340 \\
1,340 \\
1,340 \\
1,340 \\
1,340 \\
1,310 \\
1,395\end{array}$ & $\begin{array}{l}3,600 \\
3,600 \\
3,600 \\
3,800 \\
3,600 \\
3,800 \\
3,666\end{array}$ & $\begin{array}{l}1,256 \\
1,698 \\
1,920 \\
1,900 \\
2,240 \\
1,915 \\
1,810\end{array}$ & $\begin{array}{l}2,215 \\
2,210 \\
2,210 \\
2,115 \\
2,105 \\
2,105 \\
2,160\end{array}$ & $\begin{array}{l}1,450 \\
1,450 \\
1,450 \\
1,400 \\
1,360 \\
1,360 \\
1,411\end{array}$ & $\begin{array}{l}2,108 \\
2,153 \\
2,270 \\
2,128 \\
2,120 \\
2,100 \\
2,147\end{array}$ & $\begin{array}{l}680 \\
700 \\
700 \\
670 \\
670 \\
670 \\
681\end{array}$ & $\begin{array}{l}8,765 \\
0,760 \\
0,460 \\
8,595 \\
0,625 \\
8,375 \\
8,595\end{array}$ & $\begin{array}{l}2,355 \\
2,355 \\
2,300 \\
2,3100 \\
2,250 \\
2,320 \\
2,320\end{array}$ & $\begin{array}{l}3,100 \\
3,370 \\
3,370 \\
3,300 \\
3,200 \\
3,170 \\
3,205\end{array}$ & $\begin{array}{l}28,029 \\
28,496 \\
28,480 \\
28,390 \\
28,330 \\
27,755 \\
28,251\end{array}$ \\
\hline
\end{tabular}

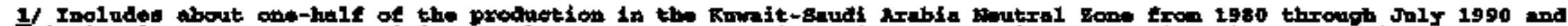

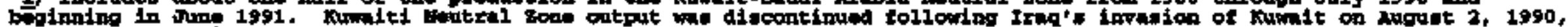

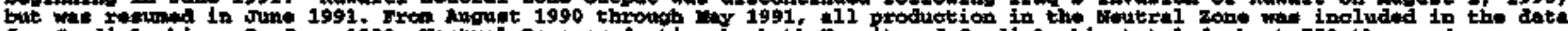

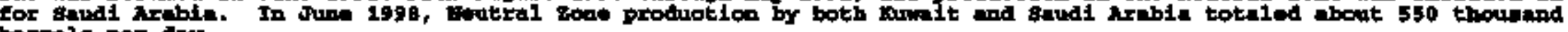
tarrele per ding.

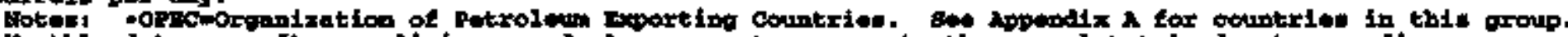

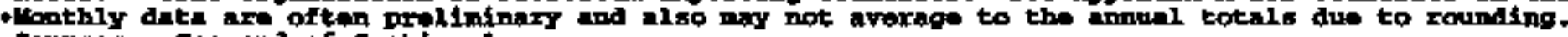

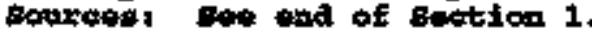




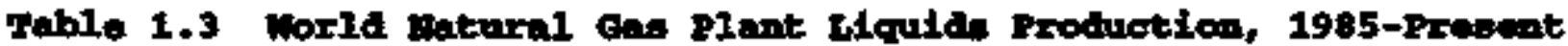

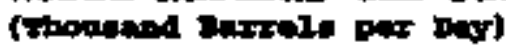

\begin{tabular}{|c|c|c|c|c|c|c|c|c|c|c|c|c|}
\hline & & Montil & Camadn & nustico & $\begin{array}{l}\text { gand } \\
\text { Nrabia }\end{array}$ & Aunate & 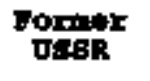 & $\begin{array}{l}\text { Onlted } \\
\text { statedy }\end{array}$ & $\begin{array}{l}\text { Burofian } \\
\text { Oaltz }\end{array}$ & anples & op:ces & Norld \\
\hline $\begin{array}{l}1985 \\
1996 \\
1987 \\
1998 \\
1999 \\
1990 \\
1991 \\
1992 \\
1993 \\
1994 \\
1995 \\
1996\end{array}$ & 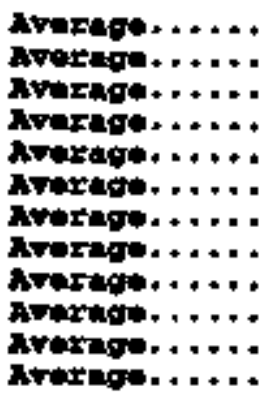 & $\begin{array}{l}120 \\
120 \\
140 \\
120 \\
130 \\
130 \\
140 \\
140 \\
145 \\
140 \\
145 \\
150\end{array}$ & $\begin{array}{l}337 \\
328 \\
367 \\
381 \\
410 \\
426 \\
431 \\
460 \\
506 \\
529 \\
581 \\
596\end{array}$ & $\begin{array}{l}271 \\
352 \\
33 \% \\
370 \\
304 \\
428 \\
457 \\
454 \\
458 \\
481 \\
447 \\
423\end{array}$ & $\begin{array}{l}375 \\
985 \\
418 \\
499 \\
503 \\
620 \\
680 \\
713 \\
704 \\
698 \\
701 \\
697\end{array}$ & $\begin{array}{l}= \\
= \\
= \\
= \\
= \\
= \\
230 \\
200 \\
200 \\
100 \\
185\end{array}$ & $\begin{array}{l}350 \\
440 \\
430 \\
450 \\
425 \\
425 \\
420 \\
=- \\
=- \\
== \\
=-\end{array}$ & $\begin{array}{l}1,609 \\
1,551 \\
1,595 \\
1,625 \\
1,546 \\
1,559 \\
1,659 \\
1,697 \\
1,736 \\
1,727 \\
1,762 \\
1,830\end{array}$ & $\begin{array}{r}645 \\
700 \\
721 \\
808 \\
051 \\
930 \\
931 \\
1,009 \\
1,040 \\
1,071 \\
1,106 \\
1,082\end{array}$ & $\begin{array}{r}800 \\
060 \\
900 \\
979 \\
1,041 \\
1,107 \\
1,113 \\
1,185 \\
1,250 \\
1,267 \\
1,301 \\
1,295\end{array}$ & $\begin{array}{r}692 \\
969 \\
1,006 \\
1,077 \\
1,186 \\
1,281 \\
1,299 \\
1,364 \\
1,435 \\
1,465 \\
1,508 \\
1,501\end{array}$ & $\begin{array}{l}3,958 \\
1,150 \\
1,279 \\
0,481 \\
0,503 \\
4,632 \\
1,027 \\
4,975 \\
5,181 \\
5,293 \\
5,485 \\
5,582\end{array}$ \\
\hline 1997 & 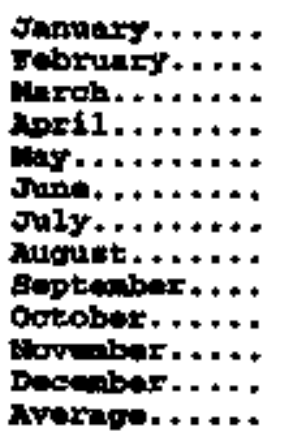 & $\begin{array}{l}145 \\
145 \\
145 \\
145 \\
145 \\
145 \\
145 \\
145 \\
145 \\
145 \\
145 \\
145 \\
145\end{array}$ & $\begin{array}{l}660 \\
645 \\
652 \\
610 \\
597 \\
595 \\
598 \\
625 \\
626 \\
645 \\
655 \\
665 \\
631\end{array}$ & $\begin{array}{l}370 \\
375 \\
395 \\
360 \\
380 \\
370 \\
370 \\
370 \\
425 \\
409 \\
405 \\
418 \\
386\end{array}$ & $\begin{array}{l}700 \\
710 \\
720 \\
725 \\
725 \\
720 \\
720 \\
730 \\
730 \\
730 \\
725 \\
735 \\
723\end{array}$ & $\begin{array}{l}200 \\
200 \\
200 \\
200 \\
200 \\
200 \\
200 \\
200 \\
200 \\
200 \\
200 \\
200 \\
200\end{array}$ & $\begin{array}{l}=- \\
=- \\
=- \\
= \\
= \\
= \\
= \\
= \\
= \\
= \\
=\end{array}$ & $\begin{array}{l}1,702 \\
1,867 \\
1,676 \\
1,824 \\
1,822 \\
1,827 \\
1,821 \\
1,831 \\
1,805 \\
1,813 \\
1,728 \\
1,773 \\
1,017\end{array}$ & $\begin{array}{l}1,095 \\
1,095 \\
1,105 \\
1,1 \pm 0 \\
1,110 \\
1,105 \\
1,105 \\
1,115 \\
1,115 \\
1,115 \\
1,110 \\
1,120 \\
1,108\end{array}$ & $\begin{array}{l}1,207 \\
1,297 \\
1,307 \\
1,312 \\
1,312 \\
1,307 \\
1,307 \\
1,317 \\
1,317 \\
1,317 \\
1,312 \\
1,322 \\
1,310\end{array}$ & $\begin{array}{l}1,465 \\
1,475 \\
1,485 \\
2,490 \\
1,490 \\
1,405 \\
1,485 \\
1,495 \\
1,495 \\
1,495 \\
1,490 \\
1,500 \\
1,480\end{array}$ & $\begin{array}{l}5,562 \\
5,627 \\
5,662 \\
5,541 \\
5,498 \\
5,471 \\
5,495 \\
5,529 \\
5,625 \\
5,614 \\
5,576 \\
5,653 \\
5,571\end{array}$ \\
\hline 1998 & 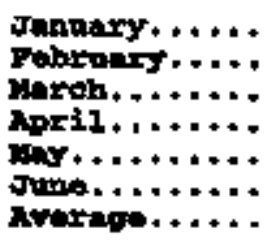 & $\begin{array}{l}145 \\
145 \\
145 \\
145 \\
145 \\
145 \\
145\end{array}$ & $\begin{array}{r}665 \\
680 \\
645 \\
645 \\
6580 \\
610 \\
637\end{array}$ & $\begin{array}{r}385 \\
420 \\
425 \\
425 \\
2430 \\
420 \\
417\end{array}$ & $\begin{array}{l}735 \\
735 \\
735 \\
725 \\
725 \\
705 \\
727\end{array}$ & $\begin{array}{l}2330 \\
1230 \\
1230 \\
2030 \\
2330 \\
230 \\
230\end{array}$ & $\begin{array}{l}=- \\
=- \\
=- \\
=- \\
=-\end{array}$ & $\begin{array}{r}1,826 \\
1,070 \\
1,846 \\
1,859 \\
21,809 \\
81,847 \\
=1,042\end{array}$ & $\begin{array}{l}1,120 \\
1,120 \\
1,120 \\
1,110 \\
1,110 \\
1,090 \\
1,112\end{array}$ & $\begin{array}{l}1,322 \\
1,322 \\
1,322 \\
1,312 \\
1,312 \\
1,292 \\
1,314\end{array}$ & $\begin{array}{l}1,500 \\
1,500 \\
1,500 \\
1,490 \\
1,490 \\
1,470 \\
1,492\end{array}$ & $\begin{array}{r}25,709 \\
25,805 \\
R 5,752 \\
25,756 \\
15,607 \\
5,636 \\
5,709\end{array}$ \\
\hline
\end{tabular}

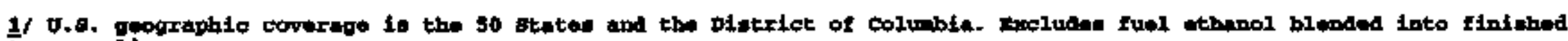
cotav ranoline.

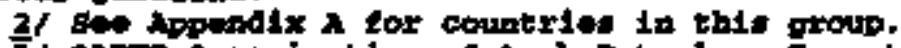

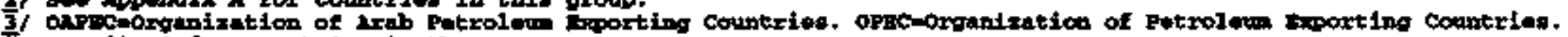

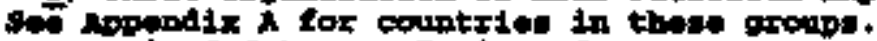

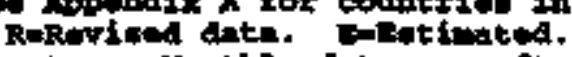

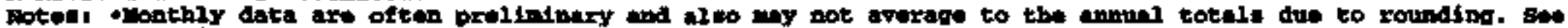

anction 1 lor bittorital Eeriel.

sevratet sus and of section 1 
Table 1.4 Morld Oil sugply, 1985-Pzentent

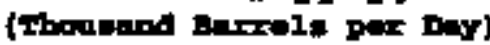

\begin{tabular}{|c|c|c|c|c|c|c|}
\hline & & 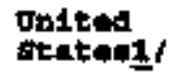 & $\begin{array}{l}\text { Porainan } \\
\text { Gulf? }\end{array}$ & Aprog/ & ortect & Worle \\
\hline $\begin{array}{l}1985 \\
1986 \\
1907 \\
1988 \\
1989 \\
1990 \\
1991 \\
1992 \\
1993 \\
1994 \\
1995 \\
1996\end{array}$ & 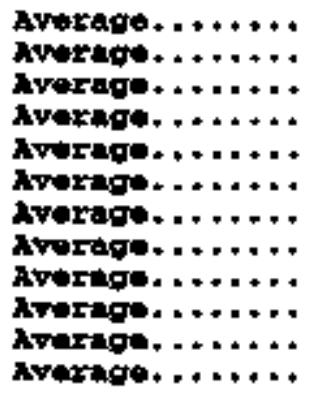 & $\begin{array}{r}11,182 \\
10,905 \\
10,648 \\
10,473 \\
9,880 \\
9,677 \\
9,683 \\
9,768 \\
9,602 \\
9,413 \\
9,400 \\
9,445\end{array}$ & $\begin{array}{l}10,320 \\
12,411 \\
12,069 \\
1,310 \\
15,733 \\
16,249 \\
15,704 \\
17,010 \\
17,792 \\
18,072 \\
18,351 \\
18,486\end{array}$ & $\begin{array}{l}11,498 \\
13,653 \\
13,992 \\
15,662 \\
16,658 \\
17,232 \\
16,747 \\
17,871 \\
19,505 \\
19,745 \\
19,069 \\
19,259\end{array}$ & $\begin{array}{l}17,151 \\
19,310 \\
19,589 \\
21,465 \\
23,323 \\
24,536 \\
24,625 \\
25,919 \\
26,610 \\
27,031 \\
27,566 \\
28,321\end{array}$ & $\begin{array}{l}59,262 \\
61,769 \\
62,427 \\
64,705 \\
65,692 \\
66,751 \\
66,632 \\
66,960 \\
67,361 \\
68,250 \\
69,860 \\
71,764\end{array}$ \\
\hline 1997 & 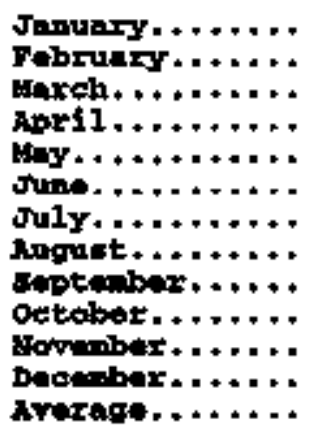 & $\begin{array}{l}9,234 \\
9,525 \\
9,428 \\
9,416 \\
9,497 \\
9,465 \\
9,465 \\
9,404 \\
9,560 \\
9,519 \\
9,457 \\
9,570 \\
9,461\end{array}$ & $\begin{array}{l}19,162 \\
19,377 \\
19,602 \\
19,762 \\
19,532 \\
19,122 \\
19,107 \\
20,127 \\
20,157 \\
20,197 \\
19,957 \\
19,573 \\
19,641\end{array}$ & $\begin{array}{l}19,898 \\
20,123 \\
20,373 \\
20,534 \\
20,344 \\
19,799 \\
19,859 \\
20,869 \\
21,089 \\
20,979 \\
20,689 \\
20,315 \\
20,407\end{array}$ & $\begin{array}{l}29,256 \\
29,531 \\
29,726 \\
29,946 \\
29,716 \\
29,326 \\
29,331 \\
30,491 \\
30,471 \\
30,611 \\
30,411 \\
30,027 \\
29,905\end{array}$ & $\begin{array}{r}73,299 \\
73,822 \\
79,791 \\
74,273 \\
73,617 \\
72,006 \\
73,016 \\
74,239 \\
74,692 \\
75,225 \\
75,063 \\
74,937 \\
74,091\end{array}$ \\
\hline $\begin{array}{l}1998 \\
1996\end{array}$ & 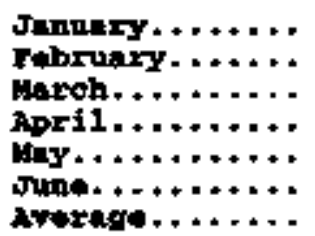 & $\begin{array}{r}9,462 \\
9,611 \\
9,442 \\
9,497 \\
7,9,340 \\
59,374 \\
\mathrm{PE} 9,452\end{array}$ & $\begin{array}{l}20,218 \\
20,670 \\
20,597 \\
20,027 \\
20,027 \\
20,352 \\
20,570\end{array}$ & $\begin{array}{r}21,000 \\
21,459 \\
21,324 \\
21,344 \\
21,494 \\
20,809 \\
21,236\end{array}$ & $\begin{array}{l}30,490 \\
30,957 \\
30,941 \\
30,849 \\
30,781 \\
30,216 \\
30,703\end{array}$ & $\begin{array}{r}75,336 \\
75,968 \\
75,717 \\
75,555 \\
274,854 \\
74,682 \\
75,344\end{array}$ \\
\hline
\end{tabular}

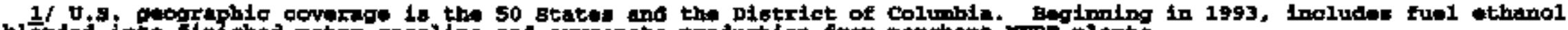

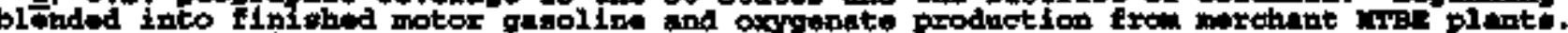

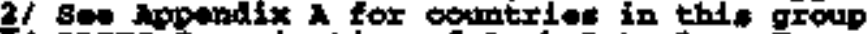

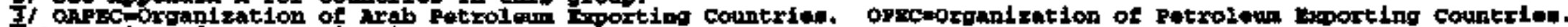

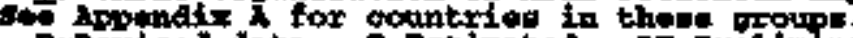

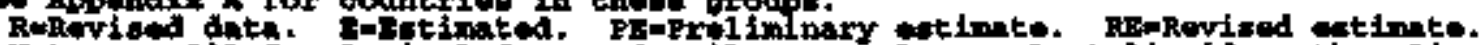

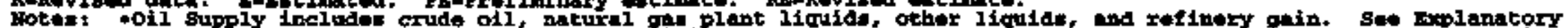

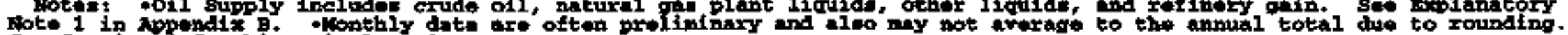

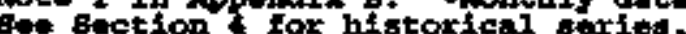

sources: Bet and of sention 1 . 
Table 1.5 oll stooks in Osco countriled, find of Pextod (riliton Burrele)

\begin{tabular}{|c|c|c|c|c|c|c|c|c|c|c|c|}
\hline & & Franot & Ittly & aecosmst & $\begin{array}{l}\text { Dnited } \\
\text { cisgion }\end{array}$ & 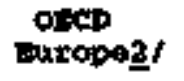 & $\begin{array}{l}\text { Jnited } \\
\text { stuters }\end{array}$ & Cannda & Jegan & otherd/ & $\begin{array}{l}\text { Total } \\
\text { Oted }\end{array}$ \\
\hline \multirow[t]{2}{*}{$\begin{array}{l}1985 \\
1986 \\
1987 \\
1988 \\
1969 \\
1990 \\
1991 \\
1992 \\
1993 \\
1994 \\
1995 \\
1996 \\
1997\end{array}$} & 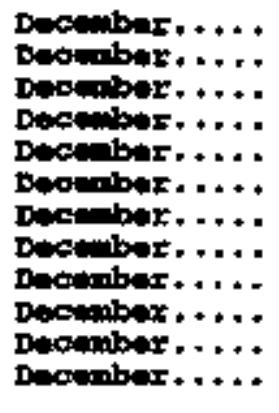 & $\begin{array}{l}139 \\
127 \\
127 \\
140 \\
138 \\
140 \\
153 \\
146 \\
158 \\
158 \\
159 \\
158\end{array}$ & $\begin{array}{l}157 \\
155 \\
169 \\
155 \\
164 \\
172 \\
160 \\
174 \\
163 \\
164 \\
162 \\
152\end{array}$ & $\begin{array}{l}253 \\
252 \\
259 \\
266 \\
271 \\
265 \\
280 \\
310 \\
309 \\
313 \\
301 \\
300\end{array}$ & $\begin{array}{l}123 \\
124 \\
121 \\
112 \\
118 \\
112 \\
119 \\
113 \\
126 \\
115 \\
107 \\
108\end{array}$ & $\begin{array}{l}1,092 \\
1,133 \\
1,190 \\
1,118 \\
1,133 \\
1,163 \\
1,181 \\
1,219 \\
1,221 \\
1,260 \\
1,228 \\
1,256\end{array}$ & $\begin{array}{l}1,519 \\
1,593 \\
1,607 \\
1,597 \\
1,581 \\
1,621 \\
1,617 \\
1,592 \\
1,647 \\
1,659 \\
1,363 \\
1,507\end{array}$ & $\begin{array}{l}113 \\
111 \\
126 \\
116 \\
114 \\
121 \\
119 \\
107 \\
105 \\
119 \\
109 \\
103\end{array}$ & $\begin{array}{l}494 \\
509 \\
540 \\
530 \\
577 \\
590 \\
606 \\
603 \\
618 \\
645 \\
630 \\
651\end{array}$ & $\begin{array}{l}66 \\
72 \\
71 \\
71 \\
71 \\
73 \\
65 \\
67 \\
69 \\
69 \\
71 \\
74\end{array}$ & $\begin{array}{l}3,284 \\
3,418 \\
3,474 \\
3,404 \\
3,476 \\
3,568 \\
3,581 \\
3,588 \\
3,661 \\
3,726 \\
3,601 \\
3,591\end{array}$ \\
\hline & 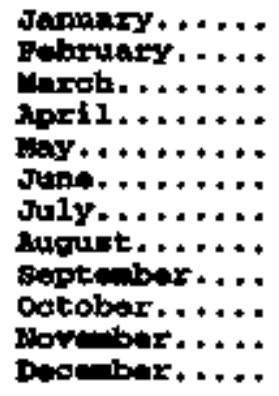 & $\begin{array}{l}156 \\
159 \\
160 \\
159 \\
153 \\
153 \\
153 \\
158 \\
157 \\
152 \\
163 \\
164\end{array}$ & $\begin{array}{l}150 \\
156 \\
160 \\
151 \\
150 \\
151 \\
150 \\
151 \\
144 \\
144 \\
150 \\
147\end{array}$ & $\begin{array}{l}306 \\
309 \\
312 \\
301 \\
311 \\
299 \\
303 \\
302 \\
291 \\
299 \\
791 \\
298\end{array}$ & $\begin{array}{l}107 \\
105 \\
109 \\
108 \\
108 \\
111 \\
112 \\
108 \\
106 \\
106 \\
106 \\
105\end{array}$ & $\begin{array}{l}1,284 \\
1,270 \\
1,273 \\
1,240 \\
1,248 \\
1,230 \\
1,230 \\
1,253 \\
1,227 \\
1,231 \\
1,251 \\
1,356\end{array}$ & $\begin{array}{l}1,501 \\
1,582 \\
1,512 \\
1,518 \\
1,561 \\
1,575 \\
1,559 \\
1,570 \\
1,592 \\
1,598 \\
1,500 \\
1,560\end{array}$ & $\begin{array}{l}106 \\
103 \\
107 \\
110 \\
104 \\
103 \\
105 \\
113 \\
108 \\
111 \\
111 \\
115\end{array}$ & $\begin{array}{l}650 \\
642 \\
650 \\
665 \\
664 \\
662 \\
670 \\
669 \\
682 \\
693 \\
699 \\
695\end{array}$ & $\begin{array}{l}80 \\
75 \\
76 \\
80 \\
81 \\
83 \\
81 \\
90 \\
77 \\
83 \\
76 \\
74\end{array}$ & $\begin{array}{l}3,617 \\
3,573 \\
3,617 \\
3,620 \\
3,658 \\
3,653 \\
3,645 \\
3,685 \\
3,687 \\
3,716 \\
3,736 \\
3,689\end{array}$ \\
\hline 1998 & 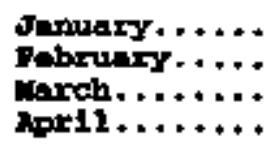 & $\begin{array}{l}163 \\
161 \\
155 \\
163\end{array}$ & $\begin{array}{l}154 \\
155 \\
146 \\
161\end{array}$ & $\begin{array}{l}290 \\
290 \\
285 \\
252\end{array}$ & $\begin{array}{l}111 \\
108 \\
109 \\
106\end{array}$ & $\begin{array}{l}1,277 \\
1,273 \\
1,251 \\
1,274\end{array}$ & $\begin{array}{l}1,576 \\
1,572 \\
1,588 \\
1,614\end{array}$ & $\begin{array}{l}112 \\
110 \\
116 \\
113\end{array}$ & $\begin{array}{l}673 \\
664 \\
655 \\
659\end{array}$ & $\begin{array}{l}78 \\
75 \\
73 \\
74\end{array}$ & $\begin{array}{l}3,716 \\
3,695 \\
3,683 \\
3,734\end{array}$ \\
\hline
\end{tabular}

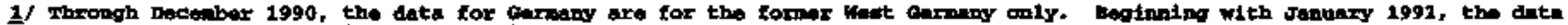

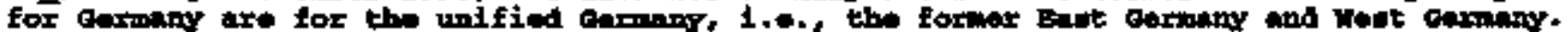

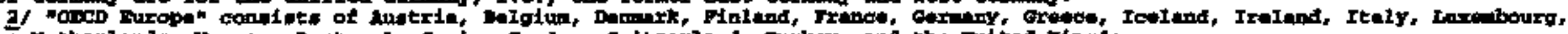

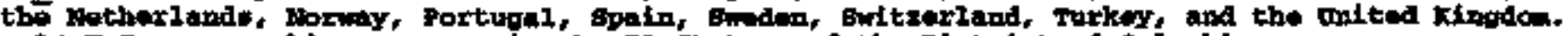

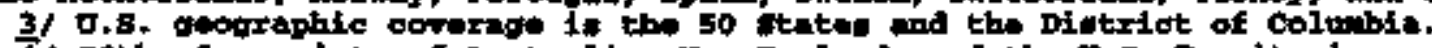

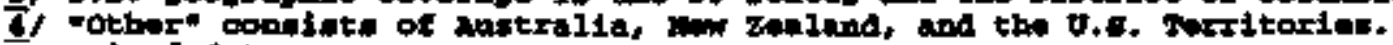

Fiporied date.

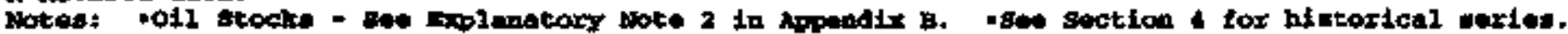

Souretia: tow and or gection 1 . 


\begin{tabular}{|c|c|c|c|}
\hline country & Comerolel & Coverimbert & Totel \\
\hline 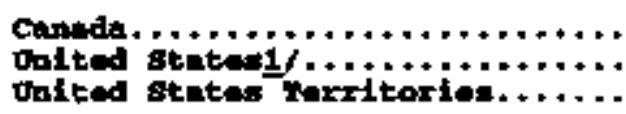 & $\begin{array}{r}116 \\
1,025 \\
25\end{array}$ & $\begin{array}{r}0 \\
563 \\
0\end{array}$ & $\begin{array}{r}116 \\
1,580 \\
25\end{array}$ \\
\hline 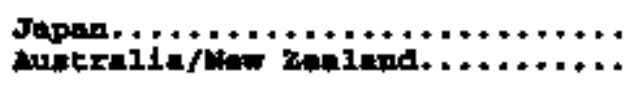 & 340 & $\begin{array}{r}315 \\
\mathbf{m}\end{array}$ & 65s \\
\hline 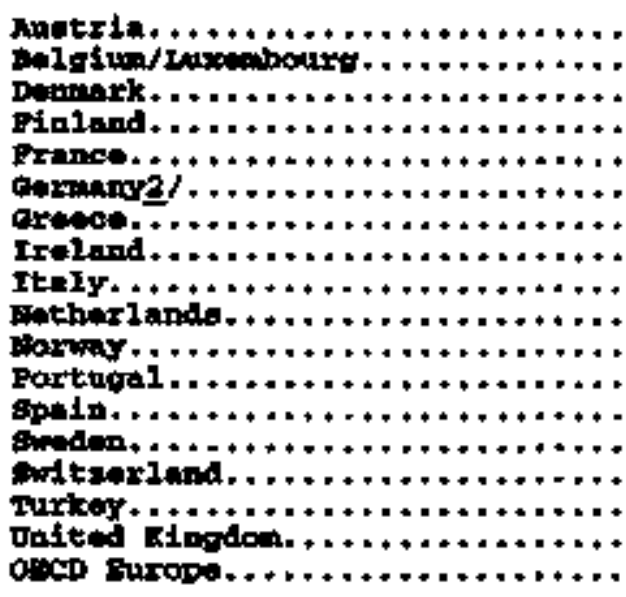 & $\begin{array}{r}22 \\
30 \\
23 \\
25 \\
155 \\
230 \\
30 \\
7 \\
140 \\
101 \\
62 \\
23 \\
101 \\
33 \\
45 \\
53 \\
109 \\
1,168\end{array}$ & $\begin{array}{r}0 \\
0 \\
0 \\
0 \\
0 \\
\mathbf{3 5} \\
\mathbf{m i n} \\
2 \\
6 \\
0 \\
0 \\
0 \\
0 \\
0 \\
0 \\
0 \\
0 \\
63\end{array}$ & $\begin{array}{r}22 \\
90 \\
23 \\
25 \\
155 \\
285 \\
30 \\
9 \\
146 \\
101 \\
62 \\
23 \\
101 \\
93 \\
15 \\
53 \\
109 \\
1,251\end{array}$ \\
\hline Totel orco.................. & 2,742 & $9 d 1$ & 3,683 \\
\hline
\end{tabular}

y/ U.s. Geofraphic covarage in the 50 stetes and the Dintrict of colunbla.

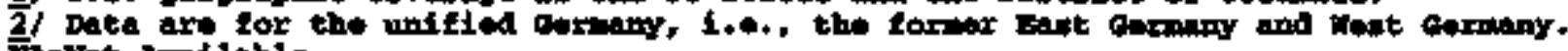
Whotiot avaluble.

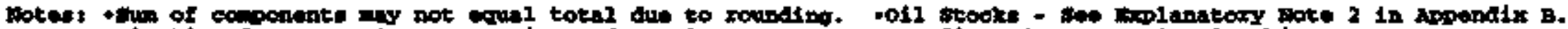

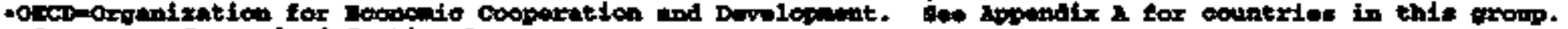

Souxces: 5to ad of section 1. 
Foble 1.7. Oi.to 011 Denend, 1985-Present (thoonned barrale par bay)

\begin{tabular}{|c|c|c|c|c|c|c|c|c|c|c|c|}
\hline & & Frange & Italy & atrinary & $\begin{array}{l}\text { Onitina } \\
\text { rimpicin }\end{array}$ & 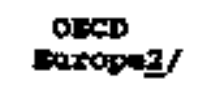 & 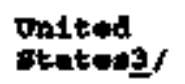 & cunade & Japan & othors/ & $\begin{array}{c}\text { Toten } \\
\text { Oted }\end{array}$ \\
\hline $\begin{array}{l}1985 \\
1986 \\
1967 \\
1968 \\
1989 \\
1990 \\
1991 \\
1992 \\
1993 \\
1994 \\
1995 \\
1996\end{array}$ & 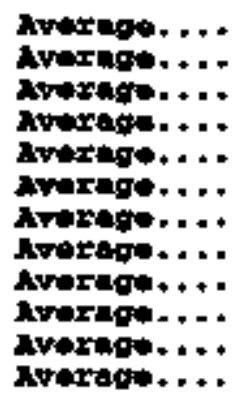 & $\begin{array}{l}1,775 \\
1,772 \\
1,709 \\
1,797 \\
1,957 \\
1,810 \\
1,935 \\
1,926 \\
1,975 \\
1,933 \\
1,996 \\
1,935\end{array}$ & $\begin{array}{l}1,717 \\
1,738 \\
1,855 \\
1,836 \\
1,930 \\
1,872 \\
1,063 \\
1,937 \\
1,852 \\
1,841 \\
2,048 \\
1,054\end{array}$ & $\begin{array}{l}2,330 \\
2,498 \\
2,424 \\
2,422 \\
2,280 \\
2,382 \\
2,828 \\
2,043 \\
2,900 \\
2,079 \\
2,875 \\
2,911\end{array}$ & $\begin{array}{l}1,634 \\
1,649 \\
1,603 \\
1,697 \\
1,739 \\
1,752 \\
1,801 \\
1,803 \\
1,815 \\
1,897 \\
1,845 \\
1,845\end{array}$ & $\begin{array}{l}11,611 \\
12,102 \\
12,255 \\
22,497 \\
12,531 \\
12,629 \\
13,391 \\
13,605 \\
13,523 \\
13,597 \\
14,120 \\
14,269\end{array}$ & $\begin{array}{l}15,726 \\
16,281 \\
16,665 \\
17,283 \\
17,325 \\
16,988 \\
16,714 \\
17,033 \\
17,737 \\
17,718 \\
17,725 \\
18,309\end{array}$ & $\begin{array}{l}1,504 \\
1,506 \\
1,540 \\
1,693 \\
1,733 \\
1,690 \\
1,622 \\
1,643 \\
1,684 \\
1,727 \\
1,755 \\
1,797\end{array}$ & $\begin{array}{l}4,344 \\
1,439 \\
4,484 \\
4,752 \\
4,983 \\
5,110 \\
5,284 \\
5,446 \\
5,401 \\
5,674 \\
5,711 \\
5,867\end{array}$ & $\begin{array}{r}976 \\
951 \\
969 \\
939 \\
998 \\
1,027 \\
1,056 \\
1,041 \\
1,119 \\
1,174 \\
1,243 \\
1,191\end{array}$ & $\begin{array}{l}34,271 \\
35,279 \\
35,911 \\
37,093 \\
37,570 \\
37,475 \\
38,067 \\
38,768 \\
38,967 \\
99,894 \\
40,354 \\
41,432\end{array}$ \\
\hline & 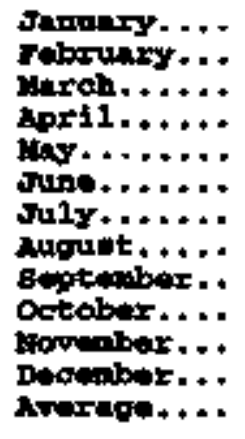 & $\begin{array}{r}2,170 \\
2,142 \\
1,901 \\
1,916 \\
1,712 \\
71,870 \\
2,077 \\
1,795 \\
1,999 \\
7,144 \\
1,731 \\
2,107 \\
1,955\end{array}$ & $\begin{array}{r}\mathrm{R} 2,029 \\
\mathrm{R} 2,115 \\
1,915 \\
\mathrm{R} 1,990 \\
\mathrm{R},, 908 \\
1,930 \\
2,020 \\
\mathrm{1} 1,799 \\
2,171 \\
2,207 \\
\mathrm{H2}, 174 \\
2,299 \\
2,045\end{array}$ & $\begin{array}{r}2,904 \\
22,653 \\
2,692 \\
3,219 \\
\mathrm{R} 2,761 \\
3,123 \\
3,074 \\
2,745 \\
3,163 \\
2,069 \\
2,692 \\
2,761 \\
\mathrm{R} 2,904\end{array}$ & $\begin{array}{r}1,850 \\
1,931 \\
1,754 \\
1,803 \\
1,712 \\
1,780 \\
1,756 \\
21,710 \\
1,821 \\
1,840 \\
11,905 \\
1,935 \\
1,799\end{array}$ & $\begin{array}{l}\mathrm{R} 14,690 \\
\mathrm{R} 14,610 \\
\mathrm{R} 13,607 \\
\mathrm{R} 14,691 \\
\mathrm{R} 13,525 \\
\mathrm{R} 14,381 \\
\mathrm{R} 14,715 \\
\mathrm{R} 13,541 \\
\mathrm{R} 15,015 \\
\mathrm{R} 15,105 \\
\mathrm{R} 14,401 \\
\mathrm{R} 14,983 \\
\mathrm{R} 14,439\end{array}$ & $\begin{array}{l}18,554 \\
18,390 \\
17,863 \\
18,559 \\
18,293 \\
18,617 \\
19,107 \\
19,565 \\
18,562 \\
19,071 \\
18,578 \\
19,250 \\
18,620\end{array}$ & $\begin{array}{r}1,936 \\
\mathrm{R} 1,857 \\
\mathrm{R1}, 755 \\
\mathrm{R1}, 721 \\
\mathrm{21}, 024 \\
\mathrm{R} 1,914 \\
1,952 \\
\mathrm{R} 4,915 \\
\mathrm{R} 1,875 \\
2,934 \\
\mathrm{R} 1,832 \\
1,076 \\
1,858\end{array}$ & $\begin{array}{r}6,294 \\
\mathrm{R} 6,736 \\
\mathrm{R} 6,149 \\
5,306 \\
\mathrm{RS}, 080 \\
5,135 \\
\mathrm{R} 5,150 \\
\mathrm{R}, 404 \\
\mathrm{R} 5,422 \\
\mathrm{R5}, 414 \\
\mathrm{R} 5,732 \\
\mathrm{T6}, 453 \\
5,711\end{array}$ & $\begin{array}{r}1,145 \\
\mathrm{Ri}, 149 \\
1,148 \\
1,181 \\
1,073 \\
1,097 \\
1,150 \\
\mathrm{x} 1,114 \\
1,166 \\
\mathrm{R} 1,140 \\
\mathrm{R} 1,152 \\
\mathrm{R1}, 146 \\
1,138\end{array}$ & 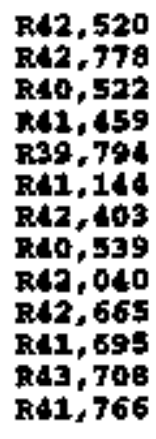 \\
\hline & 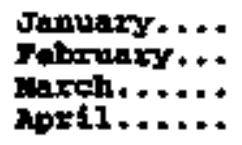 & $\begin{array}{l}2,040 \\
2,160 \\
1,982 \\
1,999\end{array}$ & $\begin{array}{l}2,030 \\
2,150 \\
2, \pm 11 \\
2,016\end{array}$ & $\begin{array}{l}2,734 \\
2,950 \\
3.153 \\
3,940\end{array}$ & $\begin{array}{l}1,779 \\
1,831 \\
1,861 \\
1,709\end{array}$ & $\begin{array}{l}14,355 \\
15,153 \\
15,125 \\
14,256\end{array}$ & $\begin{array}{l}18,256 \\
18,322 \\
18,393 \\
10,624\end{array}$ & $\begin{array}{l}1,060 \\
1,029 \\
1,061 \\
1,050\end{array}$ & $\begin{array}{l}6,109 \\
6,465 \\
5,905 \\
5,132\end{array}$ & $\begin{array}{l}1,044 \\
1,146 \\
1,228 \\
1,076\end{array}$ & $\begin{array}{r}41,633 \\
42,916 \\
42,512 \\
40,938\end{array}$ \\
\hline
\end{tabular}

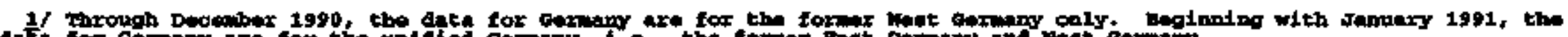

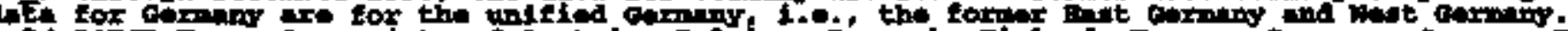

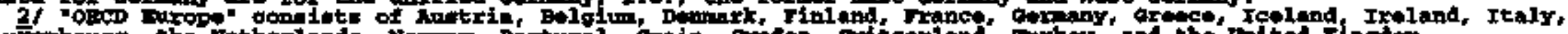

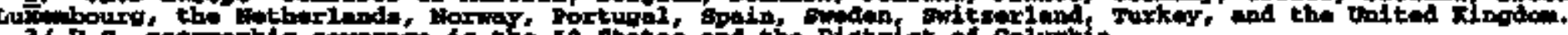

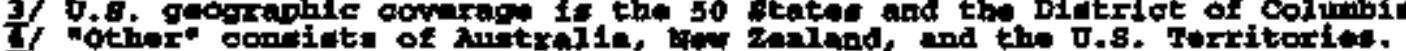

Prother dato.

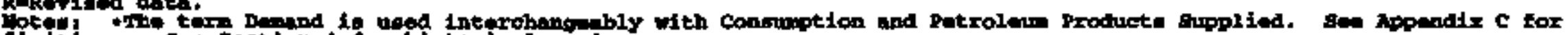

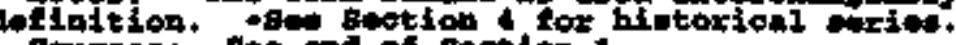

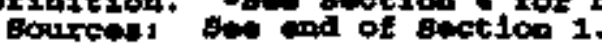




\section{Sources for Section 1 (Annual and Monthly Oil Data)}

Crode Oil Protuction, Natural Gas Liquids Production, Other Liquids, and Refinery Gain

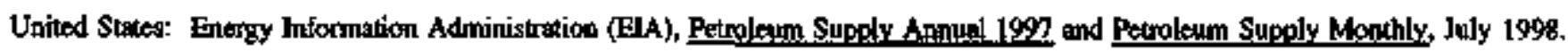

Odner Countries: Annuni data - ElA Intemational Enerovannual, various issues. Momthly data - Canala -- Maclean Hunter Publishing Conpany, Oilweek, various issues. Demunark, Mexico, Netherlands, Norway, Gemany, and all OPEC countries - Petroteutm and Energy Intelligence Weekly, Igc., Peirołeum Intellizence Weakly, various tssues. All Other Coumtrites - PennWell Pubtisting Company, Oil and Gas Joumat, various issoes. Refinery Gains: Nationtal Petrolenm Council, U,S, Petroteum Refinery Sudy, October 1986.

Oil Stocks

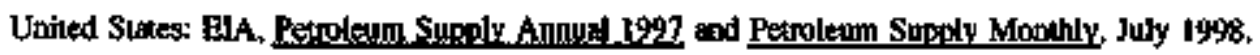

Other OECD Countries: - Oryanization for Bconemic Cooperation and Development (OECD), Oparterly Oil Sutistics and Bnerxy Balepces, various issutes. - OECD, Monthly Oil Statistics data base, 1985-1998.

Oil Deniand

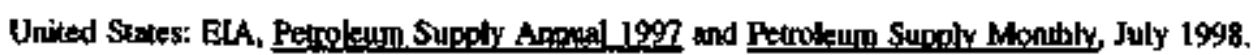

Other OECD Cowntries: • OECD, Quaterk Oil Statistics and Energy Balances, various issues. - OECD, Mondhly Oil Statistico data base. $1985-1998$. 

2. International Oil Balance Data 
Toble 2.1 Woxla oll Balande, 1994-1998

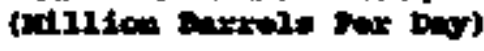

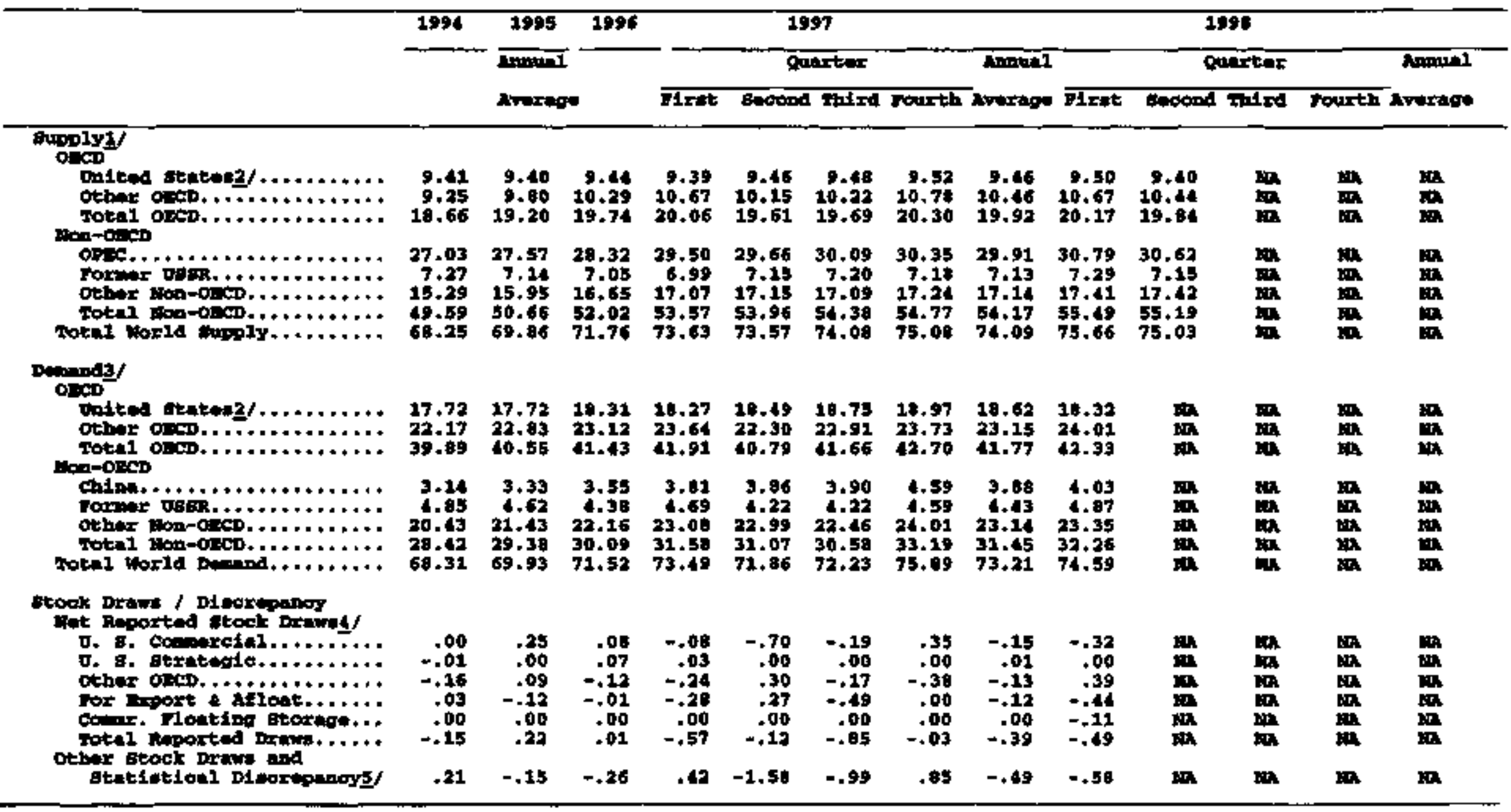

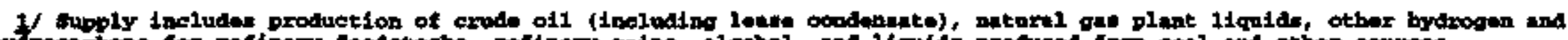

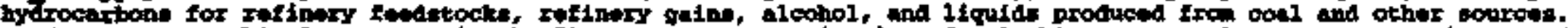

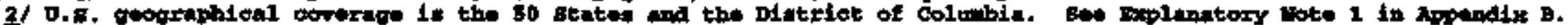

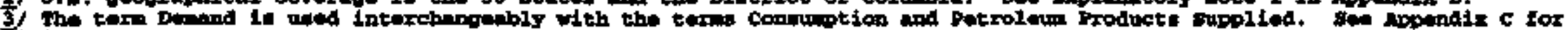
dofinition.

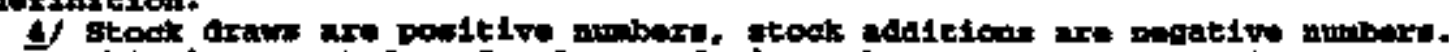

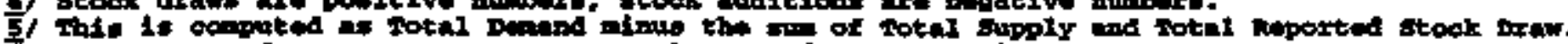

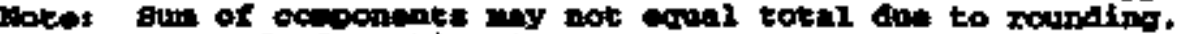

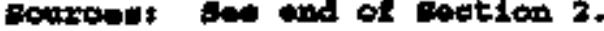


tablo 2.2 Worla oil $94001 y, 1994-1998$

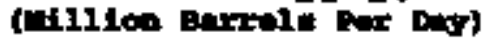

\begin{tabular}{|c|c|c|c|c|c|c|c|c|c|c|c|c|c|}
\hline & \multirow[t]{3}{*}{1994} & \multirow{3}{*}{$\frac{1995}{\text { Inmunt }}$} & \multirow[t]{3}{*}{1996} & \multicolumn{5}{|c|}{1997} & \multicolumn{4}{|c|}{1998} & \multirow{3}{*}{$\begin{array}{l}\text { Ampuel } \\
\text { Arorage }\end{array}$} \\
\hline & & & & \multicolumn{4}{|c|}{ Suarter } & \multirow{2}{*}{ 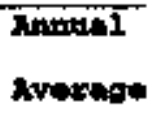 } & \multicolumn{4}{|c|}{ quncter } & \\
\hline & & & & Tiret & socond & Thixd & Tourth & & Firat: & 240004 & Thind & Fourth & \\
\hline 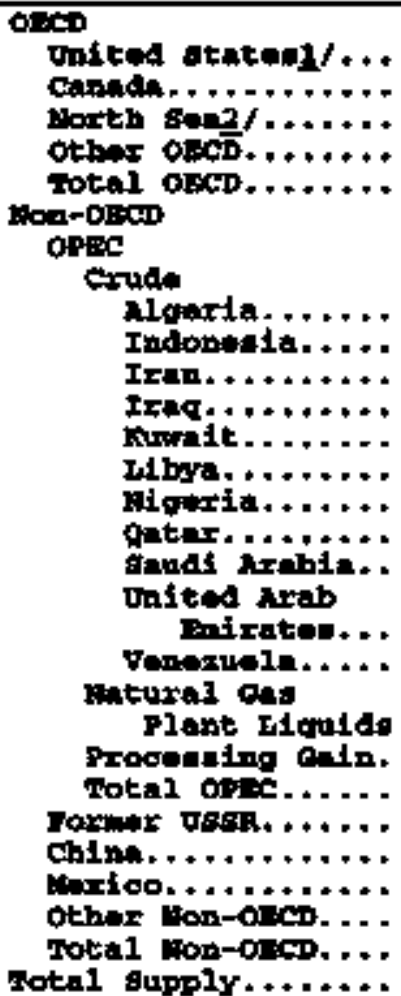 & $\begin{array}{r}1.18 \\
1.51 \\
3.62 \\
.55 \\
2.02 \\
1.38 \\
1.93 \\
. .41 \\
9.19 \\
2.19 \\
2.59 \\
1.46 \\
.06 \\
27.03 \\
7.27 \\
2.94 \\
3.17 \\
9.17 \\
49.59 \\
68.25\end{array}$ & $\begin{array}{r}1.20 \\
1.50 \\
3.64 \\
.56 \\
2.06 \\
1.39 \\
1.99 \\
.44 \\
0.29 \\
2.23 \\
2.75 \\
1.51 \\
27.06 \\
7.14 \\
2.99 \\
3.09 \\
9.66 \\
50.66 \\
69.86\end{array}$ & $\begin{array}{r}1.24 \\
1.55 \\
3.69 \\
.59 \\
2.06 \\
1.40 \\
2.19 \\
0.51 \\
0.20 \\
2.28 \\
3.05 \\
1.50 \\
.06 \\
20.32 \\
7.05 \\
3.13 \\
3.31 \\
10.22 \\
52.02 \\
71.76\end{array}$ & $\begin{array}{r}1.27 \\
1.59 \\
3.68 \\
1.13 \\
2.09 \\
1.43 \\
2.28 \\
.58 \\
8.40 \\
2.33 \\
3.19 \\
1.47 \\
.06 \\
29.50 \\
6.99 \\
3.22 \\
3.36 \\
10.49 \\
53.57 \\
73.63\end{array}$ & $\begin{array}{r}1.27 \\
1.56 \\
3.66 \\
1.07 \\
2.06 \\
1.45 \\
7.31 \\
. .63 \\
8.55 \\
2.30 \\
3.24 \\
1.49 \\
. .06 \\
29.66 \\
7.15 \\
3.24 \\
3.38 \\
10.52 \\
53.96 \\
73.57\end{array}$ & $\begin{array}{r}1.29 \\
1.52 \\
3.62 \\
1.28 \\
2.07 \\
1.45 \\
2.33 \\
.68 \\
0.63 \\
2.32 \\
3.36 \\
1.49 \\
.06 \\
90.09 \\
7.20 \\
3.19 \\
3.49 \\
10.41 \\
54.38 \\
74.08\end{array}$ & $\begin{array}{r}1.28 \\
1.52 \\
3.67 \\
1.26 \\
2.12 \\
1.45 \\
2.36 \\
.70 \\
9.67 \\
2.31 \\
3.46 \\
1.50 \\
.06 \\
30.35 \\
7.18 \\
3.15 \\
3.51 \\
10.58 \\
54.77 \\
73.08\end{array}$ & $\begin{array}{r}1.28 \\
1.55 \\
3.66 \\
1.19 \\
2.08 \\
1.45 \\
2.32 \\
. .65 \\
9.56 \\
2.32 \\
3.31 \\
1.49 \\
.06 \\
29.91 \\
7.13 \\
3.30 \\
3.44 \\
10.50 \\
54.17 \\
74.09\end{array}$ & $\begin{array}{r}1.29 \\
1.52 \\
3.63 \\
1.59 \\
2.21 \\
1.65 \\
2.29 \\
.73 \\
9.66 \\
2.49 \\
3.42 \\
1.50 \\
.06 \\
30.79 \\
7.29 \\
3.19 \\
3.57 \\
10.66 \\
55.69 \\
75.66\end{array}$ & $\begin{array}{r}1.25 \\
1.51 \\
3.77 \\
2.05 \\
2.11 \\
1.37 \\
2.22 \\
.70 \\
8.51 \\
2.35 \\
3.23 \\
1.49 \\
.06 \\
30.62 \\
7.15 \\
3.20 \\
3.57 \\
10.65 \\
55.19 \\
75.03\end{array}$ & $\begin{array}{l}\ln \\
\sin \\
\sin \\
\sin \\
\sin \\
\sin \\
\sin \\
\sin \\
\sin \\
\sin \\
\sin \\
\min \\
\min \\
\sin \\
\min \\
\sin \end{array}$ & 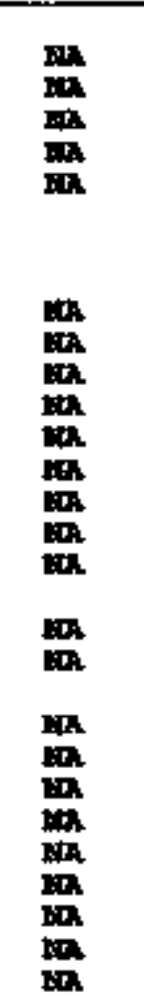 & 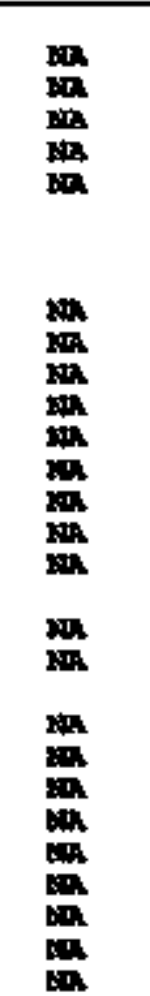 \\
\hline
\end{tabular}

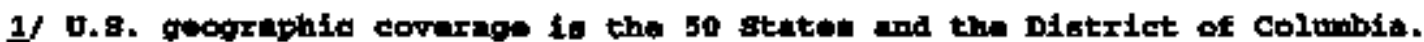

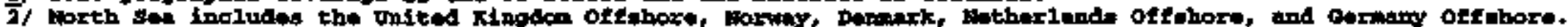

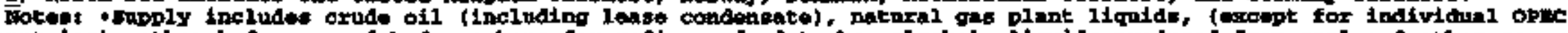

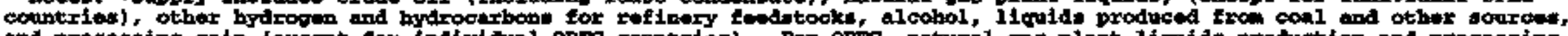

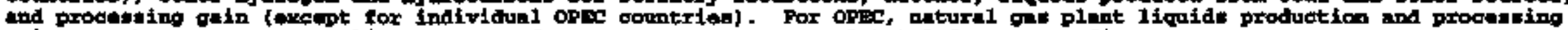

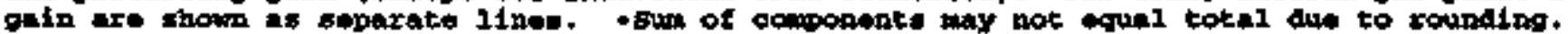

sourtets sto and of gection 2 . 


\begin{tabular}{|c|c|c|c|c|c|c|c|c|c|c|c|}
\hline & \multirow[t]{3}{*}{1994} & 1993 & \multirow[t]{3}{*}{1996} & \multicolumn{4}{|c|}{1997} & \multicolumn{4}{|c|}{1998} \\
\hline & & \multirow[t]{2}{*}{ Yonr ind } & & \multicolumn{4}{|c|}{ Qunatery } & \multicolumn{4}{|c|}{ gunxtex } \\
\hline & & & & Firtt & Second & mird & Fonret & Fixat & Second & Thixd & Folifth \\
\hline 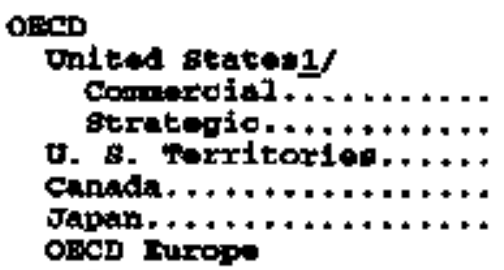 & $\begin{array}{r}1,061 \\
592 \\
26 \\
119 \\
645\end{array}$ & $\begin{array}{r}971 \\
592 \\
24 \\
109 \\
530\end{array}$ & $\begin{array}{r}941 \\
566 \\
25 \\
103 \\
651\end{array}$ & $\begin{array}{r}949 \\
569 \\
29 \\
107 \\
650\end{array}$ & $\begin{array}{r}1,012 \\
563 \\
27 \\
103 \\
662\end{array}$ & $\begin{array}{r}1,029 \\
563 \\
27 \\
108 \\
682\end{array}$ & $\begin{array}{r}997 \\
563 \\
23 \\
115 \\
685\end{array}$ & $\begin{array}{r}1,025 \\
563 \\
25 \\
116 \\
655\end{array}$ & $\begin{array}{l}\mathbf{m} \\
\mathbf{m i n} \\
\sin \\
\min \end{array}$ & $\begin{array}{l}\min _{\min } \\
\min \\
\min \end{array}$ & $\begin{array}{l}\mathbf{m} \\
\mathbf{m} \\
\mathbf{m} \\
\mathbf{m}\end{array}$ \\
\hline 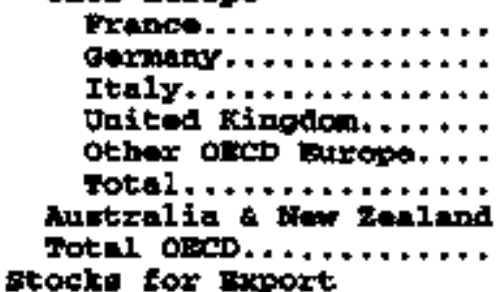 & $\begin{array}{r}158 \\
312 \\
164 \\
115 \\
490 \\
1,240 \\
3,726\end{array}$ & $\begin{array}{r}159 \\
301 \\
162 \\
107 \\
499 \\
1,228 \\
3,601\end{array}$ & $\begin{array}{r}158 \\
300 \\
152 \\
109 \\
537 \\
1,256 \\
3,591\end{array}$ & $\begin{array}{r}160 \\
312 \\
160 \\
109 \\
5312 \\
1,273 \\
53 \\
3,617\end{array}$ & $\begin{array}{r}153 \\
299 \\
151 \\
121 \\
516 \\
1,230 \\
56 \\
3,653\end{array}$ & $\begin{array}{r}157 \\
291 \\
144 \\
106 \\
528 \\
1,227 \\
5,687\end{array}$ & $\begin{array}{r}164 \\
296 \\
147 \\
105 \\
542 \\
1,256 \\
51 \\
3,689\end{array}$ & $\begin{array}{r}155 \\
285 \\
146 \\
109 \\
556 \\
1,251 \\
3.693\end{array}$ & 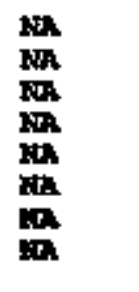 & $\begin{array}{l}\mathbf{m} \\
\mathbf{m} \\
\mathbf{m} \\
\mathbf{m} \\
\mathbf{m} \\
\mathbf{m} \\
\mathbf{m}\end{array}$ & $\begin{array}{l}\mathrm{m} \\
\mathrm{ma} \\
\mathrm{ma} \\
\mathrm{sa} \\
\mathrm{sh} \\
\mathrm{ma} \\
\mathrm{ma}\end{array}$ \\
\hline 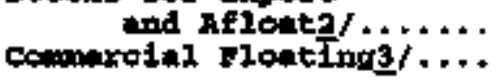 & $\begin{array}{r}935 \\
70\end{array}$ & $\begin{array}{r}980 \\
70\end{array}$ & 985 & 1,010 & $\begin{array}{r}985 \\
70\end{array}$ & $\begin{array}{r}1,030 \\
70\end{array}$ & $\begin{array}{r}1,030 \\
70\end{array}$ & $\begin{array}{r}1,070 \\
00\end{array}$ & $\sin$ & wh & $\operatorname{mat}$ \\
\hline
\end{tabular}

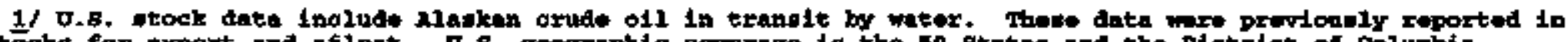

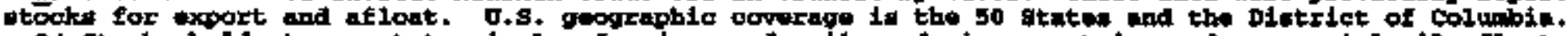

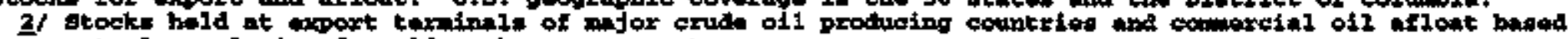

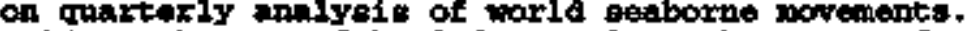

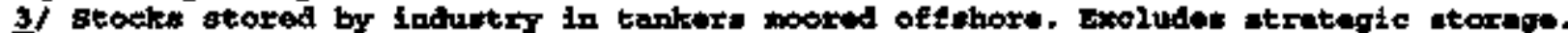

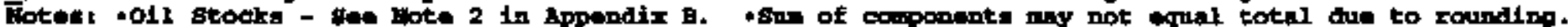

Bcuxces: tere and of Bection 2 . 


\begin{tabular}{|c|c|c|c|c|c|c|c|c|c|c|c|c|c|}
\hline & \multirow[t]{3}{*}{1994} & \multirow{3}{*}{$\frac{1995}{\text { Anmulat }}$} & \multirow[t]{3}{*}{1996} & \multicolumn{5}{|c|}{1997} & \multicolumn{5}{|c|}{2998} \\
\hline & & & & \multicolumn{4}{|c|}{ Qunterr } & \multirow{2}{*}{$\begin{array}{l}\text { Aminal } \\
\text { Araracts }\end{array}$} & \multicolumn{4}{|c|}{ Quartar } & \multirow{2}{*}{$\begin{array}{l}\text { Antual } \\
\text { Amernge }\end{array}$} \\
\hline & & & & Fintt & peora & $\operatorname{sindxd}$ & Fourth & & Firat & Exeond & Th1ra & Fouxth & \\
\hline \multicolumn{14}{|l|}{ OECD } \\
\hline 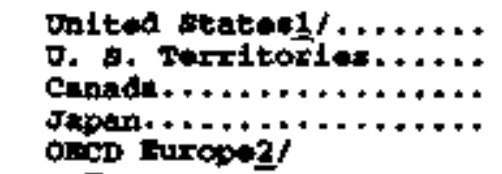 & $\begin{array}{r}17.72 \\
1.26 \\
1.73 \\
5.67\end{array}$ & $\begin{array}{r}17.72 \\
.25 \\
1.76 \\
5.71\end{array}$ & $\begin{array}{r}10.31 \\
+26 \\
1+90 \\
5.97\end{array}$ & $\begin{array}{r}18.27 \\
.20 \\
1.81 \\
6.39\end{array}$ & $\begin{array}{r}18+49 \\
+17 \\
5+12\end{array}$ & $\begin{array}{r}18.75 \\
.19 \\
1.91 \\
5.43\end{array}$ & $\begin{array}{r}18.97 \\
.20 \\
1.88 \\
5.97\end{array}$ & $\begin{array}{r}18.62 \\
.19 \\
\pm .86 \\
5.71\end{array}$ & $\begin{array}{r}18.32 \\
.21 \\
1.86 \\
6.15\end{array}$ & $\lim _{\ln }$ & $\begin{array}{l}\operatorname{man} \\
\min \end{array}$ & $\begin{array}{l}\operatorname{con} \\
\operatorname{sa} \\
\sin \end{array}$ & $\begin{array}{l}\operatorname{cR} \\
\tan \\
\tan \\
\min \end{array}$ \\
\hline 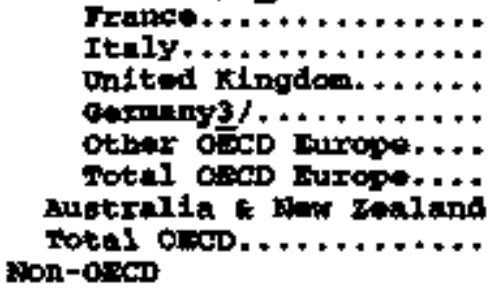 & $\begin{array}{r}1.63 \\
1.84 \\
2.84 \\
2.80 \\
5.21 \\
13.60 \\
.92 \\
39.89\end{array}$ & $\begin{array}{r}1.90 \\
2.05 \\
2.84 \\
2.80 \\
5.45 \\
14.12 \\
40.59\end{array}$ & $\begin{array}{r}1.93 \\
2.06 \\
1.85 \\
2.91 \\
5.92 \\
14.27 \\
.93 \\
41.43\end{array}$ & $\begin{array}{r}2.03 \\
2.02 \\
1.04 \\
2.75 \\
5.65 \\
14.29 \\
.95 \\
41.91\end{array}$ & $\begin{array}{r}1.93 \\
1.94 \\
1.76 \\
3.03 \\
5.62 \\
14.19 \\
40.94\end{array}$ & $\begin{array}{r}1.96 \\
1.99 \\
1.76 \\
2.99 \\
5.72 \\
14.43 \\
41.96\end{array}$ & $\begin{array}{r}2.00 \\
2.23 \\
1.83 \\
2.84 \\
5.94 \\
14.83 \\
4.94 \\
42.70\end{array}$ & $\begin{array}{r}1.96 \\
2.04 \\
1.00 \\
2.90 \\
5.74 \\
14.44 \\
41.77\end{array}$ & $\begin{array}{r}2.06 \\
2.10 \\
1.02 \\
2.95 \\
5.94 \\
14.86 \\
12.93\end{array}$ & 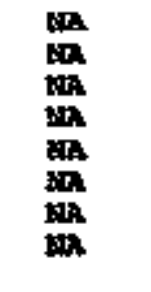 & 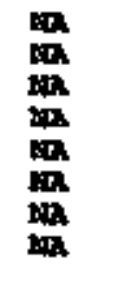 & 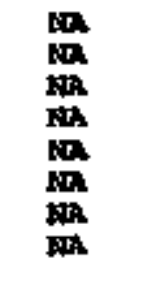 & $\begin{array}{l}\mathbf{m}_{\mathbf{n}} \\
\mathbf{m} \\
\mathbf{m} \\
\mathbf{m} \\
\mathbf{m} \\
\mathbf{m} \\
\mathbf{m}\end{array}$ \\
\hline 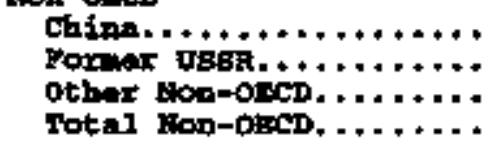 & $\begin{array}{r}3.14 \\
4.85 \\
20.43 \\
28.42\end{array}$ & $\begin{array}{r}3.33 \\
4.62 \\
21.43 \\
29.38\end{array}$ & $\begin{array}{r}3.55 \\
4.38 \\
22.16 \\
30.09\end{array}$ & $\begin{array}{r}3.91 \\
4.69 \\
23.08 \\
31.58\end{array}$ & $\begin{array}{r}3.66 \\
4.22 \\
22.99 \\
31.07\end{array}$ & $\begin{array}{r}3.90 \\
4.22 \\
22.46 \\
30.58\end{array}$ & $\begin{array}{r}4.59 \\
4.59 \\
24.01 \\
33.19\end{array}$ & $\begin{array}{r}3.98 \\
2.43 \\
31.14\end{array}$ & $\begin{array}{r}4.03 \\
4.07 \\
23.35 \\
32.76\end{array}$ & $\begin{array}{l}\mathrm{m} \\
\mathrm{m} \\
\mathrm{m}\end{array}$ & $\underset{m}{\min }$ & $\begin{array}{l}\sin \\
\mathbf{m i} \\
\min \end{array}$ & $\begin{array}{l}\mathrm{Ma} \\
\mathrm{M} \\
\mathrm{MR}\end{array}$ \\
\hline Total World Damend....... & 68.31 & 69.53 & 71.52 & 73.49 & 71.06 & 72.23 & 75.89 & 73.21 & 74.59 & sa & $\mathbf{m}$ & 致 & $\mathbf{m}$ \\
\hline
\end{tabular}

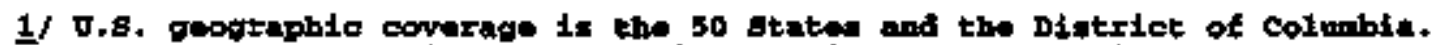

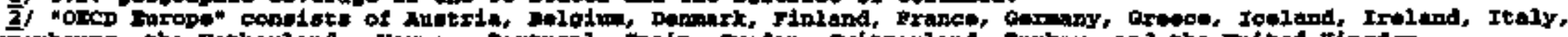

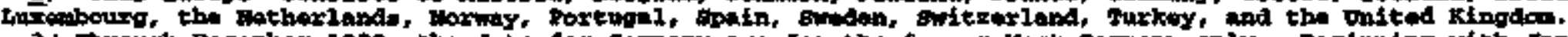

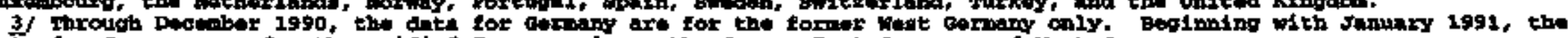

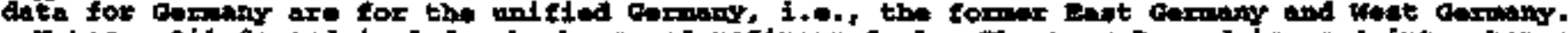

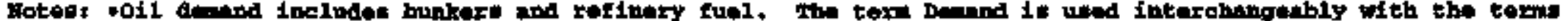

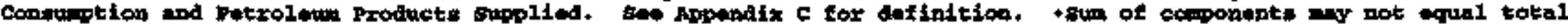
due to roungling

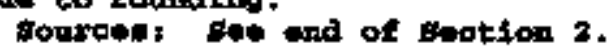




\section{Sources for Section 2 (International Oil Balance Data)}

Crude Oil Production, Natural Gas Liquids Production, Other Liquids, and Refinery Onin

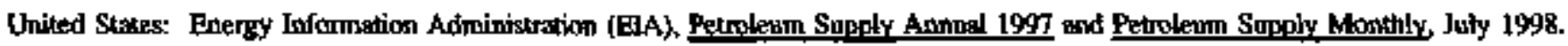

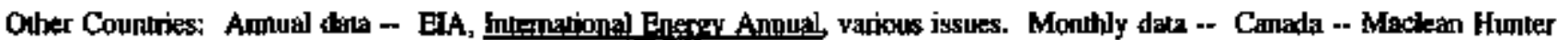
Publishing Company, 0ilwett, various issues. Denmart, Mexion, Netherlends, Norway, Germany, and all OPEC countries - Petrokeum and Fnergy Intelligence Weekly, Inc., Petroleum Intelliperce Weetly, varions issues. Other Countries -- PennWcll Publishing

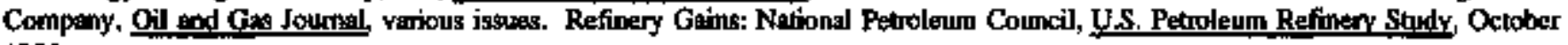
1986.

\section{Oil Stocks}

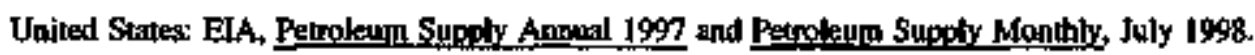

Other OECD Countries: * Organization for Economic Cooperalion and Devekpment (OECD), Onartedy Oil. Statistics and Energy Balances, various issures. - OECD, Momhly Ol Statistics data base, 1990-1998. Stocks for Export and Afloal and Conmercial Fkating Stocks - Petroleum Economics Limited, Oil Industry Developments, various issues.

Oil Demiand

United States: EIA, Petrokum Supply Annual 1997 and Petroleum Supoly Mondhy, July 1998.

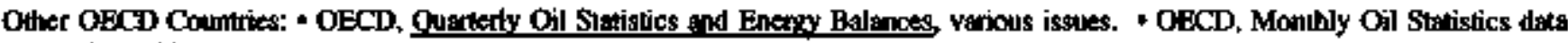
base. $1990-1998$.

OPEC Countries: ElA, Intematingal Epersy Annual, various issuos and Shont-Temm Energy Outiook, vacious issues.

Other coumtries: ElA, International Energy Anpual, various issues and Shont-Tegm. Energy Outlock, various issues. 
3. International Oll Imports 


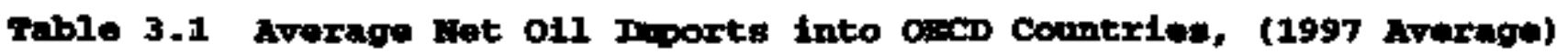
(billion Burmels par Day)

\begin{tabular}{|c|c|c|c|c|c|c|c|c|c|c|c|}
\hline & \multirow{2}{*}{ Total } & \multicolumn{6}{|c|}{ OrCD Itroge } & \multicolumn{4}{|c|}{ OneD Ontalde ruropo } \\
\hline & & Total & $\begin{array}{l}\text { United } \\
\text { singdom }\end{array}$ & Brance & Corgengy 1 / & Italy & $\begin{array}{c}\text { Otber } \\
\text { Europkaf }\end{array}$ & $\begin{array}{l}\text { Dnited } \\
\text { atratesest }\end{array}$ & Camada & Japan & Otbers 4 \\
\hline 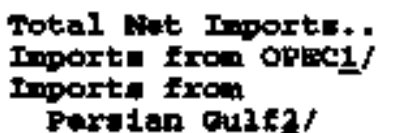 & $\begin{array}{l}21.819 \\
16.460\end{array}$ & $\begin{array}{l}7.340 \\
6.264\end{array}$ & $\begin{array}{r}-0.968 \\
.199\end{array}$ & $\begin{array}{r}1.769 \\
+999\end{array}$ & $\begin{array}{r}2.804 \\
.645\end{array}$ & $\begin{array}{l}1.755 \\
1.353\end{array}$ & $\begin{array}{l}1.940 \\
3.168\end{array}$ & $\begin{array}{l}9.158 \\
4.542\end{array}$ & $\begin{array}{r}-0.816 \\
.313\end{array}$ & $\begin{array}{r}5.619 \\
4.699\end{array}$ & $\begin{array}{r}0.318 \\
.642\end{array}$ \\
\hline 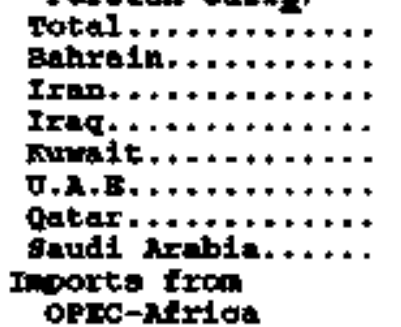 & $\begin{array}{r}9.932 \\
.025 \\
1.436 \\
.057 \\
1.065 \\
1.523 \\
. .377 \\
5.050\end{array}$ & $\begin{array}{r}3.470 \\
.001 \\
.942 \\
.336 \\
.249 \\
.009 \\
.000 \\
1.933\end{array}$ & $\begin{array}{l}.094 \\
.000 \\
.000 \\
.000 \\
.031 \\
.001 \\
.000 \\
.062\end{array}$ & $\begin{array}{l}.617 \\
.000 \\
.127 \\
.076 \\
.017 \\
.000 \\
.000 \\
.394\end{array}$ & $\begin{array}{l}. \$ 57 \\
.000 \\
.023 \\
.001 \\
.012 \\
.001 \\
.000 \\
.120\end{array}$ & $\begin{array}{l}.503 \\
.000 \\
.250 \\
.033 \\
.000 \\
.000 \\
.000 \\
-291\end{array}$ & $\begin{array}{r}2.019 \\
.000 \\
.533 \\
.223 \\
.109 \\
.007 \\
.000 \\
1.067\end{array}$ & $\begin{array}{r}1.747 \\
-.002 \\
.000 \\
.099 \\
.283 \\
=.003 \\
.004 \\
1.406\end{array}$ & $\begin{array}{l}.120 \\
.032 \\
.014 \\
.000 \\
.000 \\
.074\end{array}$ & $\begin{array}{r}4.304 \\
.023 \\
.461 \\
.015 \\
.557 \\
1.441 \\
.364 \\
1.441\end{array}$ & $\begin{array}{l}.291 \\
.002 \\
.000 \\
.003 \\
.006 \\
.076 \\
.008 \\
.195\end{array}$ \\
\hline 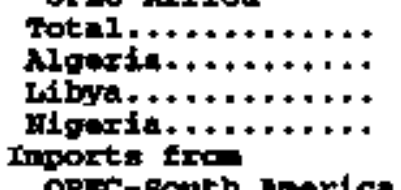 & $\begin{array}{l}3.825 \\
1.043 \\
1.269 \\
1.513\end{array}$ & $\begin{array}{r}2.572 \\
.683 \\
1.269 \\
.620\end{array}$ & $\begin{array}{l}.074 \\
.029 \\
.051 \\
.004\end{array}$ & $\begin{array}{l}.282 \\
.131 \\
.060 \\
.091\end{array}$ & $\begin{array}{l}.442 \\
.095 \\
.246 \\
.101\end{array}$ & $\begin{array}{l}.745 \\
.086 \\
.609 \\
.050\end{array}$ & $\begin{array}{r}1.030 \\
.353 \\
.304 \\
.373\end{array}$ & $\begin{array}{l}.979 \\
.205 \\
.000 \\
.694\end{array}$ & $\begin{array}{l}.093 \\
.059 \\
.000 \\
.024\end{array}$ & $\begin{array}{l}.036 \\
.014 \\
.023\end{array}$ & $\begin{array}{r}.156 \\
.003 \\
.000 \\
.153\end{array}$ \\
\hline 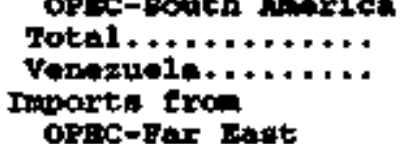 & $\begin{array}{l}2.155 \\
2.155\end{array}$ & .206 & $\begin{array}{l}.031 \\
.031\end{array}$ & $\begin{array}{l}.040 \\
.000\end{array}$ & .046 & .012 & $\begin{array}{l}.117 \\
.217\end{array}$ & $\begin{array}{l}1.758 \\
3.758\end{array}$ & $\begin{array}{r}.109 \\
.109\end{array}$ & .003 & $\begin{array}{r}.080 \\
.000\end{array}$ \\
\hline $\begin{array}{l}\text { Totnd } \ldots \ldots \ldots \ldots \ldots \\
\text { Indonerian } \ldots \ldots \ldots\end{array}$ & .572 & $\begin{array}{l}.017 \\
.017\end{array}$ & .000 & $\begin{array}{l}.000 \\
.000\end{array}$ & $\begin{array}{l}.000 \\
.000\end{array}$ & .014 & $\begin{array}{l}.002 \\
.002\end{array}$ & $\begin{array}{l}.057 \\
.057\end{array}$ & $\begin{array}{l}.000 \\
.000\end{array}$ & .300 & $\begin{array}{l}.118 \\
.118\end{array}$ \\
\hline
\end{tabular}

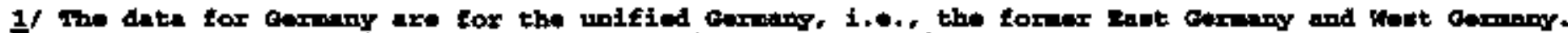

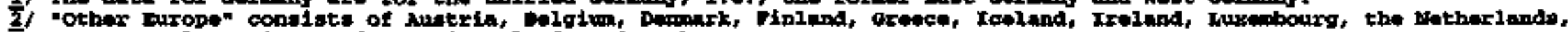

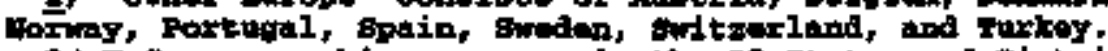

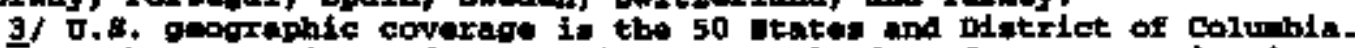

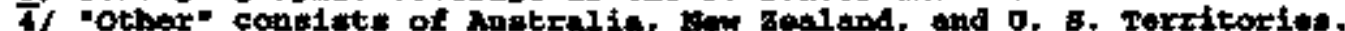

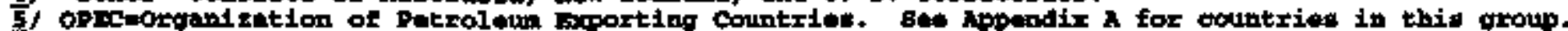

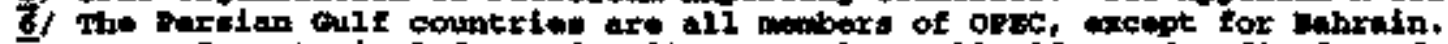

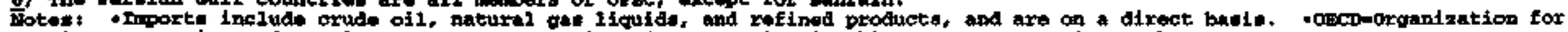

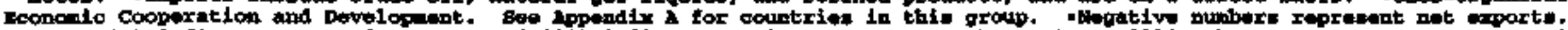

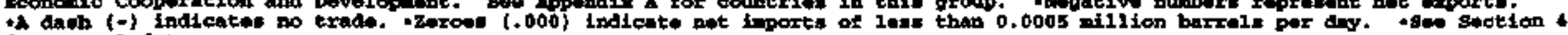
tor anmoun lata.

Soliteres sen wind of Beation 3. 
Table 3.2 wet ofl mporte Into Osed countries, (1et garter 1998) (nillion marrels Der Dwr)

\begin{tabular}{|c|c|c|c|c|c|c|c|c|c|c|c|}
\hline & \multirow{2}{*}{ Totel } & \multicolumn{6}{|c|}{ Onect raxopu } & \multicolumn{4}{|c|}{ ODED Oatside fuxcope } \\
\hline & & Total & $\begin{array}{l}\text { tonited } \\
\text { Rinidom }\end{array}$ & France & Goxmany 1 & Italy & $\begin{array}{l}\text { other } \\
\text { Euxopezy }\end{array}$ & $\begin{array}{l}\text { Onited } \\
\text { steten } 3 /\end{array}$ & Canade & Japan & otheres/ \\
\hline 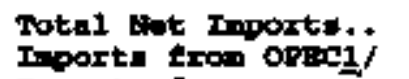 & $\begin{array}{l}21.725 \\
16.642\end{array}$ & $\begin{array}{l}7.496 \\
6.479\end{array}$ & $\begin{array}{r}-1.018 \\
.205\end{array}$ & $\begin{array}{r}1.011 \\
.942\end{array}$ & $\begin{array}{r}2.729 \\
.599\end{array}$ & $\begin{array}{l}1.849 \\
1.388\end{array}$ & $\begin{array}{l}2.126 \\
3.355\end{array}$ & $\begin{array}{l}9.022 \\
4.405\end{array}$ & $\begin{array}{r}-0.899 \\
.260\end{array}$ & $\begin{array}{l}5.777 \\
4.746\end{array}$ & $\begin{array}{r}0.329 \\
.7352\end{array}$ \\
\hline 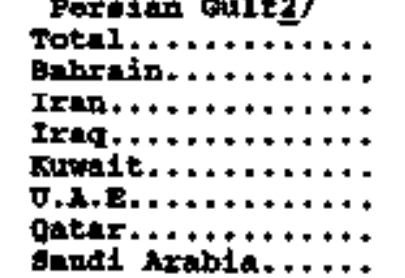 & $\begin{array}{r}10.133 \\
.026 \\
1.308 \\
. .506 \\
1.158 \\
1.527 \\
.520 \\
5.089\end{array}$ & $\begin{array}{r}3.565 \\
.011 \\
.783 \\
.446 \\
.298 \\
.002 \\
.000 \\
2.024\end{array}$ & $\begin{array}{l}107 \\
.000 \\
.000 \\
.00 \\
.025 \\
.000 \\
.000 \\
.003\end{array}$ & $\begin{array}{l}.647 \\
.004 \\
.158 \\
.005 \\
.032 \\
.002 \\
.000 \\
.007\end{array}$ & $\begin{array}{l}.113 \\
.000 \\
.011 \\
.000 \\
.013 \\
.000 \\
.000 \\
.009\end{array}$ & $\begin{array}{l}.531 \\
.007 \\
.192 \\
.044 \\
.002 \\
.000 \\
.000 \\
.286\end{array}$ & $\begin{array}{r}2.167 \\
.000 \\
.423 \\
.319 \\
.227 \\
.000 \\
.000 \\
1.199\end{array}$ & $\begin{array}{r}1.792 \\
+.003 \\
.000 \\
.056 \\
.261 \\
.002 \\
.000 \\
1.476\end{array}$ & $\begin{array}{l}.048 \\
.000 \\
.000 \\
.000 \\
.048\end{array}$ & $\begin{array}{l}. .41 \\
.015 \\
.514 \\
.000 \\
.591 \\
1.161 \\
.494 \\
1.365\end{array}$ & $\begin{array}{l}.267 \\
.003 \\
.010 \\
.002 \\
.009 \\
.062 \\
.026 \\
.175\end{array}$ \\
\hline 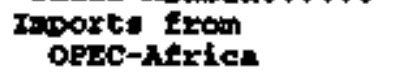 & & & & & & & & & & & \\
\hline 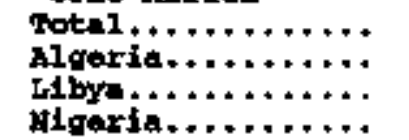 & $\begin{array}{l}3.922 \\
1.143 \\
1.280 \\
1.499\end{array}$ & $\begin{array}{l}2.691 \\
1.785 \\
1.290 \\
.627\end{array}$ & $\begin{array}{l}.074 \\
.076 \\
.042 \\
.016\end{array}$ & $\begin{array}{l}.298 \\
.127 \\
.040 \\
.132\end{array}$ & $\begin{array}{l}.447 \\
.123 \\
.259 \\
.064\end{array}$ & $\begin{array}{l}.792 \\
.093 \\
.614 \\
.085\end{array}$ & $\begin{array}{r}1.080 \\
.425 \\
.325 \\
.330\end{array}$ & $\begin{array}{l}.931 \\
.281 \\
.000 \\
.650\end{array}$ & $\begin{array}{l}.113 \\
.081 \\
.032\end{array}$ & $\begin{array}{l}.005 \\
.005 \\
.000\end{array}$ & $\begin{array}{r}.181 \\
-.009 \\
.000 \\
.190\end{array}$ \\
\hline $\begin{array}{l}\text { morte excich } \\
\text { opex-South anerica }\end{array}$ & & & & & & & & & & & \\
\hline 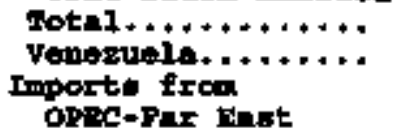 & $\begin{array}{l}2.080 \\
2.080\end{array}$ & $\begin{array}{l}.185 \\
.185\end{array}$ & .029 & $\begin{array}{l}.000 \\
.000\end{array}$ & $\begin{array}{l}.028 \\
.028\end{array}$ & $\begin{array}{l}.026 \\
.026\end{array}$ & $\begin{array}{l}.108 \\
.108\end{array}$ & $\begin{array}{l}1.643 \\
1.643\end{array}$ & $\begin{array}{l}.098 \\
.098\end{array}$ & $\begin{array}{l}.000 \\
.000\end{array}$ & $\begin{aligned} .154 \\
.150\end{aligned}$ \\
\hline 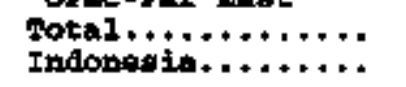 & $\begin{array}{l}.532 \\
.532\end{array}$ & .048 & $\overline{-}$ & .000 & .001 & .047 & .000 & $\begin{array}{l}.036 \\
.036\end{array}$ & .000 &. .315 & $\begin{array}{l}.133 \\
.133\end{array}$ \\
\hline
\end{tabular}

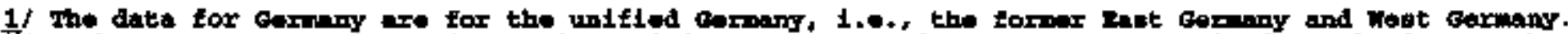

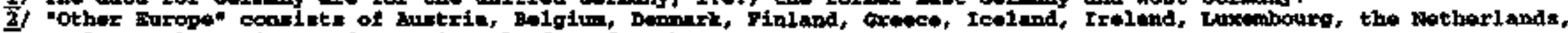

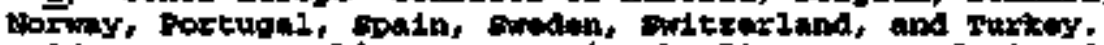

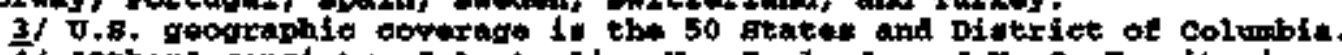

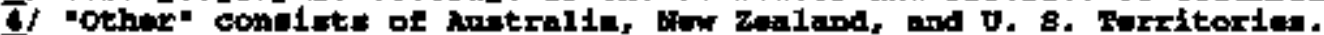

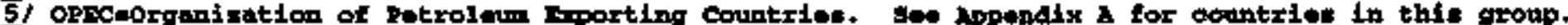

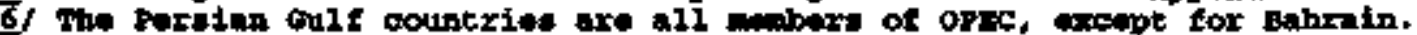

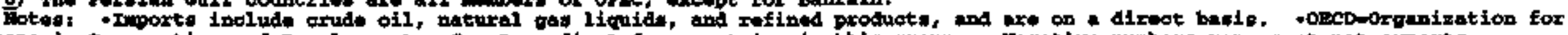

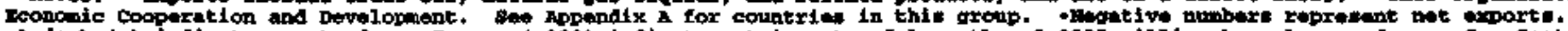

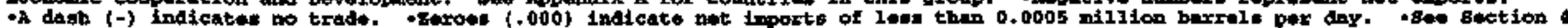
For annuel dats.

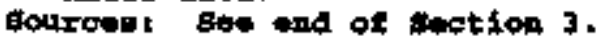


Tablo 3.3 wot oil I ports 1nto oxpo Conterles, (4th Quarter 1997) (Hillion Basciele per Dar)

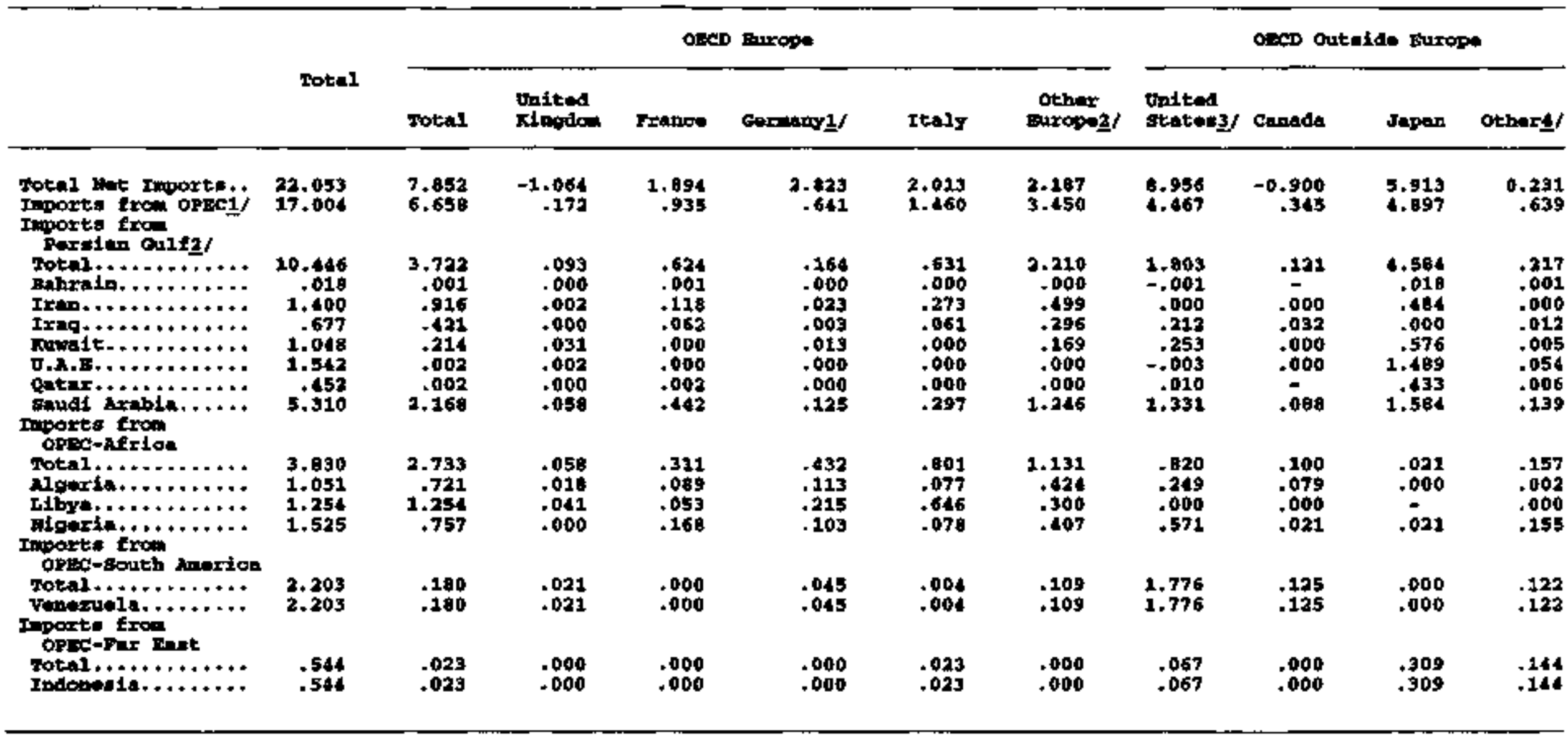

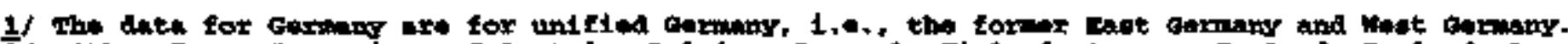

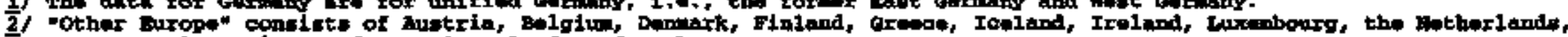
Nomisy, Portubal, Bpuln, Swhion, Switzerlam, and Turkey.

3/ U.\$. geographio comernge is the 50 staten and Diftrigt of columbis.

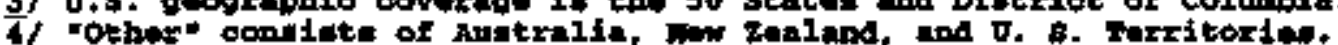

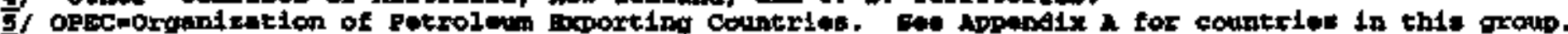

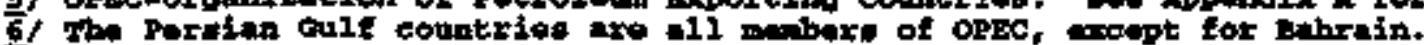

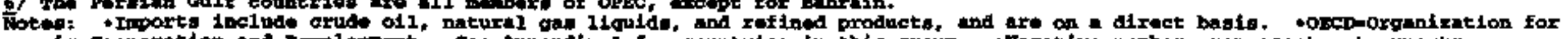

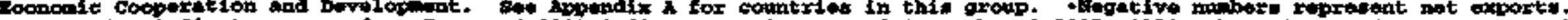

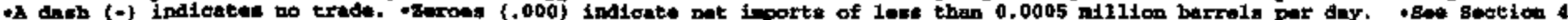
for tonmel dinte.

sources: gee end of seotion 3 . 
Tablo 3.4 Gid Total Het Inporta (Nowt Recent 12 Wonthe) (niliton sarrele par Day)

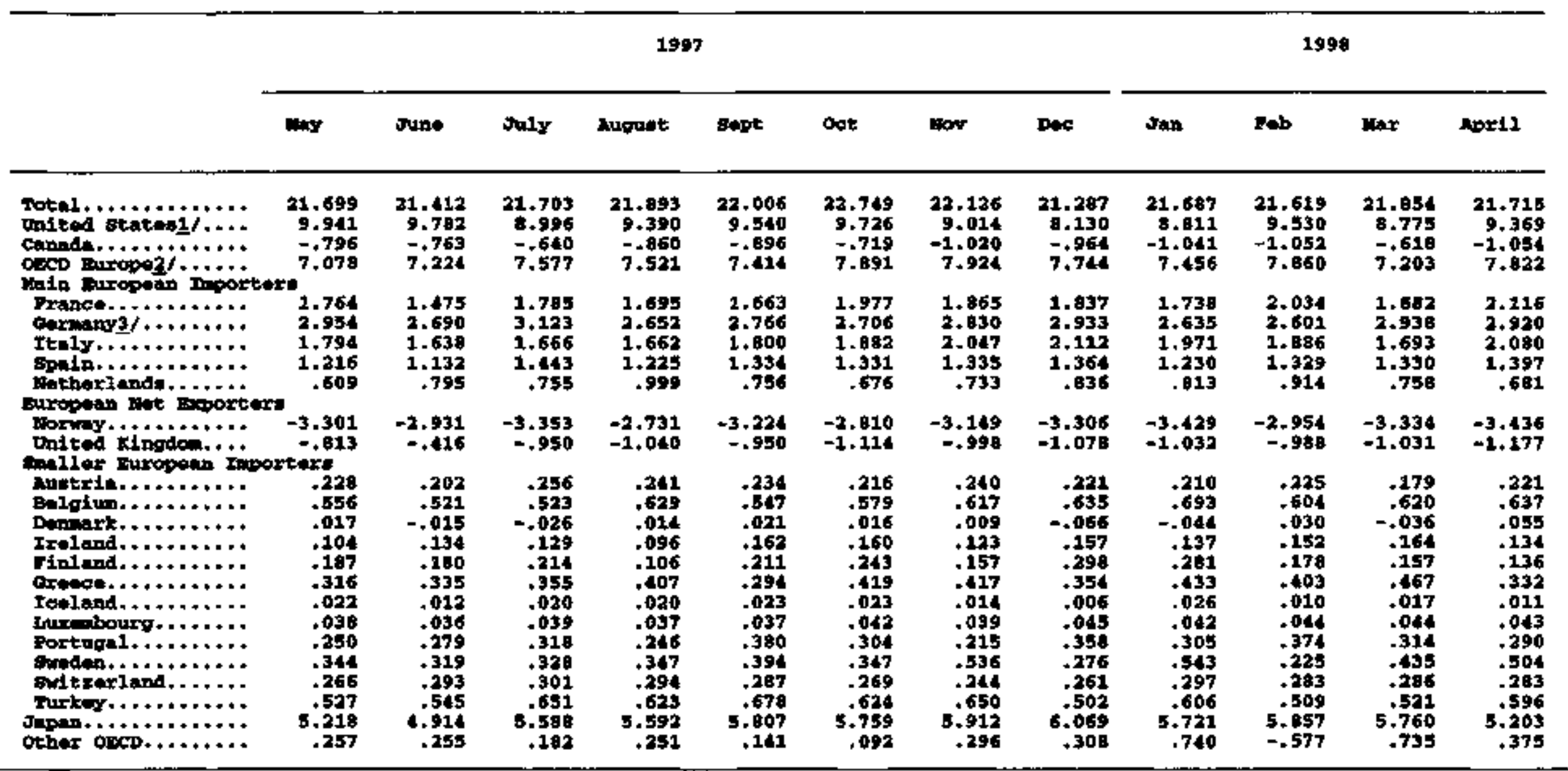

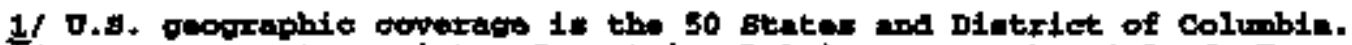

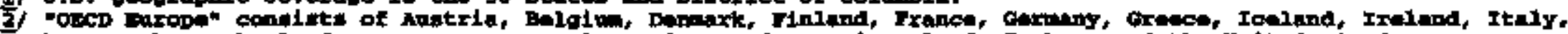

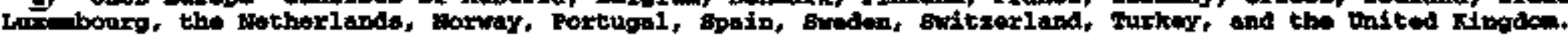

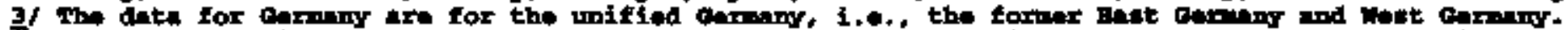

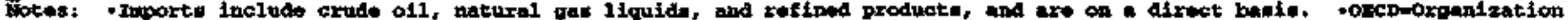

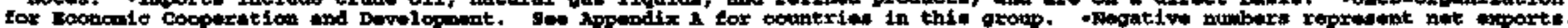

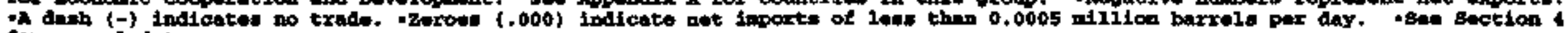
Lor anminal ditt.

tomate! Bes and of section 3 . 
Table 3.5 Oaco mport: from OPBC (wost Recent 12 wointhe)

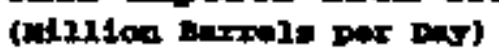

\begin{tabular}{|c|c|c|c|c|c|c|c|c|c|c|c|c|}
\hline & \multicolumn{8}{|c|}{1997} & \multicolumn{4}{|c|}{1998} \\
\hline & Way & Jume & raly & musut & Eept & oot & Bov & Deo & $\operatorname{San}$ & Pab & war & $\mathbf{p p r} 11$ \\
\hline 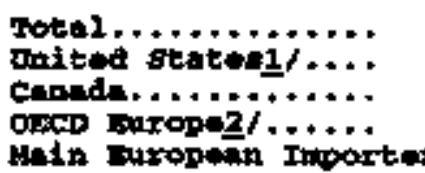 & $\begin{array}{r}16.171 \\
4.972 \\
.241 \\
6.152 \\
+\end{array}$ & $\begin{array}{r}15.568 \\
4.962 \\
.255 \\
5.666\end{array}$ & $\begin{array}{r}15.819 \\
1.112 \\
.296 \\
6.264\end{array}$ & $\begin{array}{r}26.453 \\
4.804 \\
.459 \\
6.250\end{array}$ & $\begin{array}{r}16.987 \\
4.841 \\
.282 \\
6.721\end{array}$ & $\begin{array}{r}17.273 \\
1.800 \\
.409 \\
6.695\end{array}$ & $\begin{array}{r}16.958 \\
4.378 \\
.260 \\
6.735\end{array}$ & $\begin{array}{r}16.877 \\
4.140 \\
.362 \\
6.545\end{array}$ & $\begin{array}{r}16.447 \\
4.268 \\
.223 \\
6.383\end{array}$ & $\begin{array}{r}16.719 \\
4.217 \\
.289 \\
6.921\end{array}$ & $\begin{array}{r}16.769 \\
4.712 \\
.269 \\
6.176\end{array}$ & $\begin{array}{r}16.650 \\
4.756 \\
.304 \\
6.652\end{array}$ \\
\hline 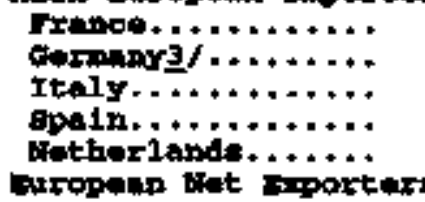 & $\begin{array}{r}.753 \\
.683 \\
1.484 \\
.703 \\
.787\end{array}$ & $\begin{array}{r}.824 \\
.581 \\
1.202 \\
.693 \\
.699\end{array}$ & $\begin{array}{r}1.020 \\
.657 \\
1.379 \\
.764 \\
.735\end{array}$ & $\begin{array}{r}.797 \\
.698 \\
1.276 \\
.722 \\
.946\end{array}$ & $\begin{array}{r}1.084 \\
.636 \\
1.379 \\
.064 \\
.711\end{array}$ & $\begin{array}{r}1.131 \\
.649 \\
1.353 \\
.820 \\
.006\end{array}$ & $\begin{array}{r}.846 \\
.647 \\
1.491 \\
.794 \\
.829\end{array}$ & $\begin{array}{r}.826 \\
.627 \\
1.535 \\
1.032 \\
.061\end{array}$ & $\begin{array}{r}.897 \\
.601 \\
1.336 \\
.873 \\
.035\end{array}$ & $\begin{array}{r}1.071 \\
.595 \\
1.624 \\
.917 \\
.896\end{array}$ & $\begin{array}{r}-891 \\
-571 \\
1+228 \\
+872 \\
-854\end{array}$ & $\begin{array}{r}1.171 \\
.601 \\
1.518 \\
.668 \\
.723\end{array}$ \\
\hline 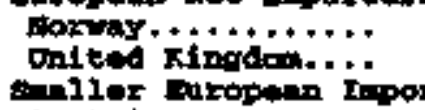 & $\begin{array}{l}.000 \\
.199 \\
\text { rteri }\end{array}$ & $\begin{array}{l}.000 \\
.219\end{array}$ & .000 & .000 & .000 & $\begin{array}{r}.007 \\
.170\end{array}$ & .009 & $\begin{array}{l}.005 \\
-129\end{array}$ & $\begin{array}{l}.000 \\
.176\end{array}$ & $\begin{array}{l}.000 \\
.140\end{array}$ & $\begin{array}{l}.000 \\
.293\end{array}$ & $\begin{array}{l}.000 \\
.153\end{array}$ \\
\hline 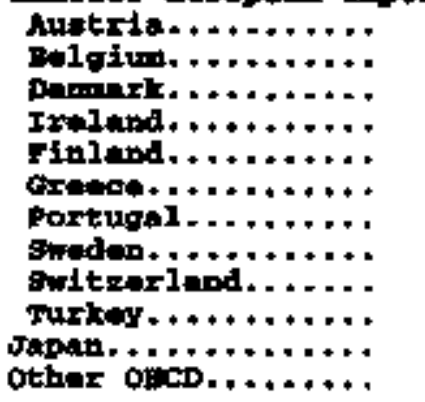 & $\begin{array}{l}.148 \\
.315 \\
.049 \\
.000 \\
.010 \\
.286 \\
.180 \\
.093 \\
.087 \\
.377 \\
4.175 \\
.631\end{array}$ & $\begin{array}{l}.058 \\
.309 \\
.033 \\
.000 \\
.000 \\
.294 \\
.179 \\
.092 \\
.093 \\
.390 \\
1.977 \\
.709\end{array}$ & $\begin{array}{r}.176 \\
.244 \\
.006 \\
.000 \\
.000 \\
.296 \\
.207 \\
.067 \\
.094 \\
.342 \\
4.366 \\
.483\end{array}$ & $\begin{array}{r}.130 \\
.326 \\
.027 \\
.000 \\
.000 \\
.330 \\
.159 \\
.089 \\
.101 \\
.458 \\
.417 \\
.523\end{array}$ & $\begin{array}{r}.133 \\
.352 \\
.067 \\
.000 \\
.008 \\
.328 \\
.291 \\
.025 \\
.107 \\
.497 \\
.453 \\
.591\end{array}$ & $\begin{array}{r}.161 \\
.351 \\
.024 \\
.000 \\
.007 \\
.367 \\
.135 \\
.086 \\
.111 \\
.517 \\
4.775 \\
.514\end{array}$ & $\begin{array}{r}.154 \\
.318 \\
.019 \\
.000 \\
.007 \\
.398 \\
.164 \\
.170 \\
.098 \\
.543 \\
.799 \\
.686\end{array}$ & $\begin{array}{r}.130 \\
.315 \\
.020 \\
.000 \\
.032 \\
.319 \\
.205 \\
.008 \\
.093 \\
.000 \\
5.112 \\
.717\end{array}$ & $\begin{array}{r}.084 \\
.330 \\
.024 \\
.0 \\
.000 \\
.401 \\
.147 \\
.147 \\
.102 \\
.430 \\
4.732 \\
.841\end{array}$ & $\begin{array}{r}.156 \\
.376 \\
.027 \\
- \\
.000 \\
.351 \\
.248 \\
.077 \\
.108 \\
.435 \\
4.589 \\
.701\end{array}$ & $\begin{array}{r}.075 \\
.257 \\
.024 \\
4 \\
.010 \\
.343 \\
.154 \\
.127 \\
.082 \\
.404 \\
4.902 \\
.709\end{array}$ & $\begin{array}{r}.157 \\
.326 \\
.033 \\
- \\
.000 \\
.284 \\
.153 \\
.126 \\
.102 \\
.436 \\
+.368 \\
.571\end{array}$ \\
\hline
\end{tabular}

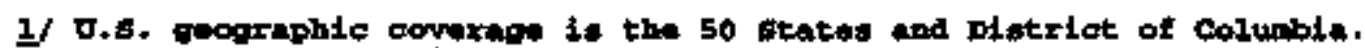

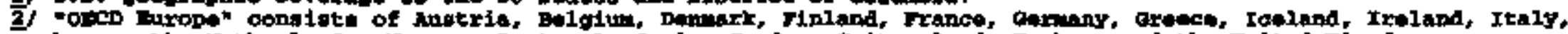

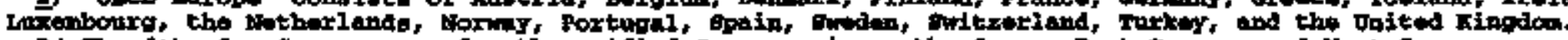

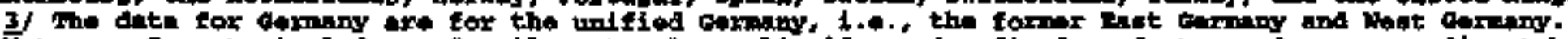

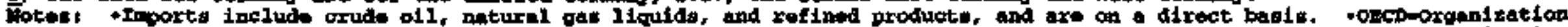

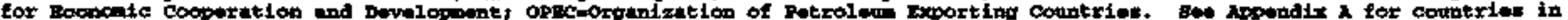

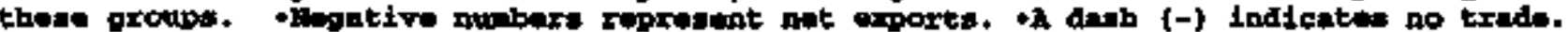

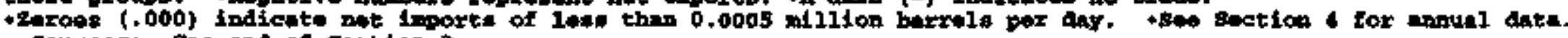
sorres: ste and of arotion 3 . 
Teble 3.6 C.cob Inoorts Erow Porsian Gulf (Nost Recent 12 wonths)

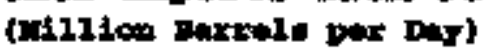

\begin{tabular}{|c|c|c|c|c|c|c|c|c|c|c|c|c|}
\hline & \multicolumn{8}{|c|}{1997} & \multicolumn{4}{|c|}{1998} \\
\hline & tany & rune & Tuls & Muguet & Bupt & oct & wer & Dese & $\operatorname{Tan}$ & Bab & Mar & April \\
\hline 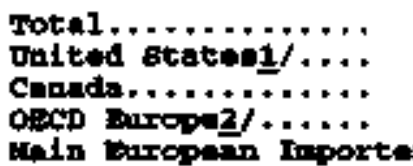 & $\begin{array}{r}9.622 \\
1.701 \\
.137 \\
3.641\end{array}$ & $\begin{array}{r}\$ .946 \\
1.759 \\
.136 \\
3.184\end{array}$ & $\begin{array}{r}9.412 \\
1.740 \\
.066 \\
3.333\end{array}$ & $\begin{array}{r}9.930 \\
1.651 \\
.198 \\
3.641\end{array}$ & $\begin{array}{r}10.331 \\
1.915 \\
.071 \\
3.962\end{array}$ & $\begin{array}{r}10.773 \\
1.913 \\
.168 \\
4.000\end{array}$ & $\begin{array}{r}10.372 \\
1.745 \\
.065 \\
3.114\end{array}$ & $\begin{array}{r}10.192 \\
1.749 \\
.129 \\
3.355\end{array}$ & $\begin{array}{r}9.932 \\
1.724 \\
.007 \\
9.472\end{array}$ & $\begin{array}{r}10.251 \\
1.691 \\
.074 \\
3.063\end{array}$ & $\begin{array}{r}10.168 \\
1.950 \\
.067 \\
3.389\end{array}$ & $\begin{array}{r}10.313 \\
1.979 \\
.070 \\
3.957\end{array}$ \\
\hline 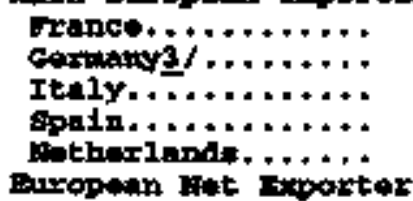 & $\begin{array}{r}.555 \\
.153 \\
.646 \\
.413 \\
.642\end{array}$ & $\begin{array}{r}.563 \\
.192 \\
.526 \\
.297 \\
.515\end{array}$ & $\begin{array}{l}.640 \\
.177 \\
.618 \\
.318 \\
.562\end{array}$ & $\begin{array}{l}.644 \\
.202 \\
.571 \\
.358 \\
.679\end{array}$ & $\begin{array}{l}.857 \\
.164 \\
.629 \\
.428 \\
.488\end{array}$ & $\begin{array}{l}.042 \\
.205 \\
.645 \\
.411 \\
.640\end{array}$ & $\begin{array}{l}.525 \\
.155 \\
.603 \\
.336 \\
.739\end{array}$ & $\begin{array}{l}.503 \\
.131 \\
.645 \\
.373 \\
.636\end{array}$ & $\begin{array}{l}.671 \\
.101 \\
.476 \\
.410 \\
.574\end{array}$ & $\begin{array}{l}.769 \\
.124 \\
.686 \\
.394 \\
.702\end{array}$ & $\begin{array}{l}.513 \\
.115 \\
.446 \\
.370 \\
.679\end{array}$ & $\begin{array}{l}.851 \\
.178 \\
.688 \\
.471 \\
.450\end{array}$ \\
\hline 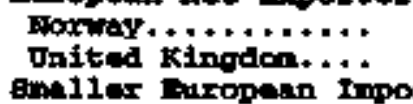 & $\begin{array}{r}.000 \\
.107 \\
\text { rteras }\end{array}$ & .000 & $\begin{array}{l}.000 \\
.123\end{array}$ & $\begin{array}{l}.000 \\
.091\end{array}$ & $\begin{array}{l}.000 \\
.115\end{array}$ & $\begin{array}{l}.000 \\
.081\end{array}$ & $\begin{array}{l}.000 \\
.129\end{array}$ & $\begin{array}{l}.000 \\
.069\end{array}$ & $\begin{array}{r}.000 \\
.078\end{array}$ & $\begin{array}{l}.000 \\
.060\end{array}$ & $\begin{array}{l}.000 \\
.180\end{array}$ & $\begin{array}{r}.000 \\
.102\end{array}$ \\
\hline 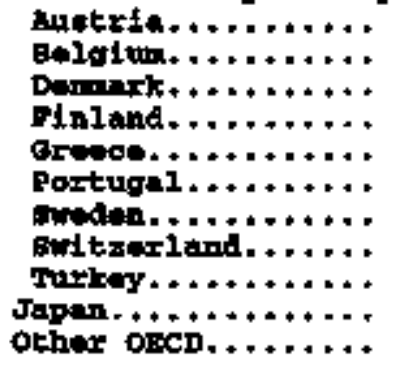 & $\begin{array}{r}.057 \\
.285 \\
.040 \\
.000 \\
.254 \\
.161 \\
.064 \\
.014 \\
.289 \\
3.816 \\
.327\end{array}$ & $\begin{array}{l}.015 \\
.250 \\
.000 \\
.000 \\
.243 \\
.138 \\
.067 \\
.014 \\
.249 \\
. .505 \\
.361\end{array}$ & $\begin{array}{l}.000 \\
.228 \\
.000 \\
.000 \\
.264 \\
.069 \\
.067 \\
.009 \\
.178 \\
3.909 \\
.364\end{array}$ & $\begin{array}{l}.019 \\
.292 \\
.000 \\
.000 \\
.207 \\
.094 \\
.065 \\
.009 \\
.330 \\
3.972 \\
.267\end{array}$ & $\begin{array}{r}.063 \\
.331 \\
.023 \\
.000 \\
.292 \\
.190 \\
.000 \\
.005 \\
.378 \\
4.147 \\
.236\end{array}$ & $\begin{array}{r}.017 \\
.332 \\
.000 \\
.000 \\
.328 \\
.051 \\
.066 \\
.014 \\
.338 \\
.518 \\
.175\end{array}$ & $\begin{array}{l}.063 \\
.302 \\
.000 \\
.000 \\
.358 \\
.005 \\
.136 \\
.005 \\
.359 \\
.526 \\
.222\end{array}$ & $\begin{array}{l}.039 \\
.280 \\
.000 \\
.000 \\
.299 \\
.121 \\
.000 \\
.000 \\
.751 \\
.705 \\
.255\end{array}$ & $\begin{array}{r}.000 \\
.326 \\
.000 \\
.000 \\
.369 \\
.078 \\
+129 \\
.004 \\
.257 \\
+.453 \\
.336\end{array}$ & $\begin{array}{r}.040 \\
.251 \\
.000 \\
+000 \\
.301 \\
.173 \\
.067 \\
.000 \\
+287 \\
+.312 \\
.912\end{array}$ & $\begin{array}{r}.017 \\
.239 \\
.000 \\
.000 \\
.312 \\
.136 \\
.108 \\
.009 \\
.267 \\
4.545 \\
.217\end{array}$ & $\begin{array}{r}.072 \\
.311 \\
.008 \\
.000 \\
.251 \\
.121 \\
.111 \\
.017 \\
.327 \\
.098 \\
.209\end{array}$ \\
\hline
\end{tabular}

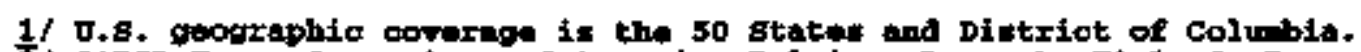

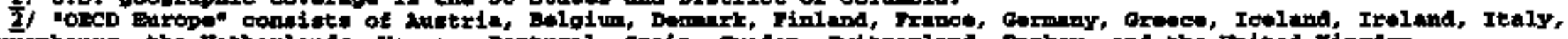

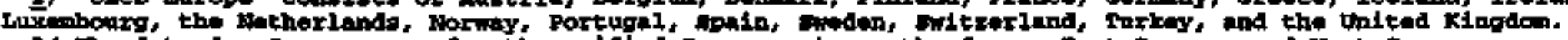

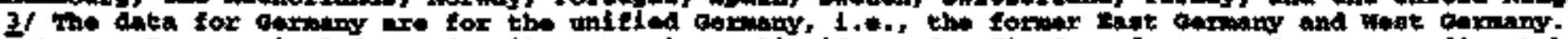

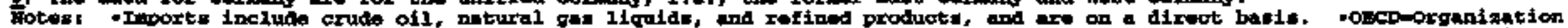

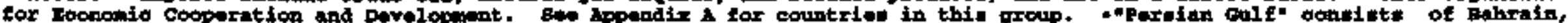

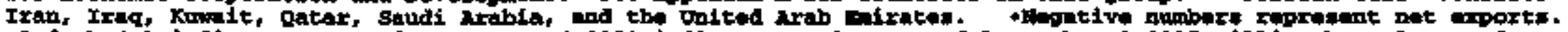

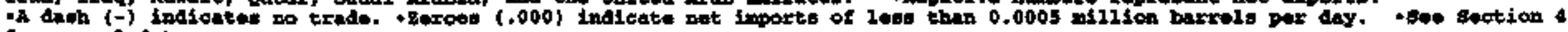
for anmul date.

foluroser 8 end of soction 3. 


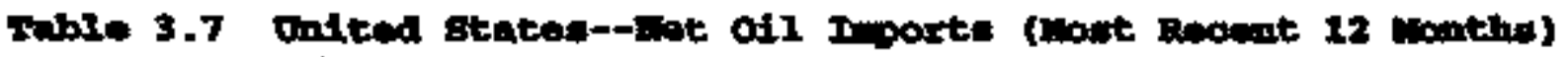

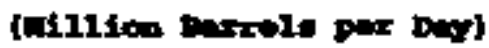

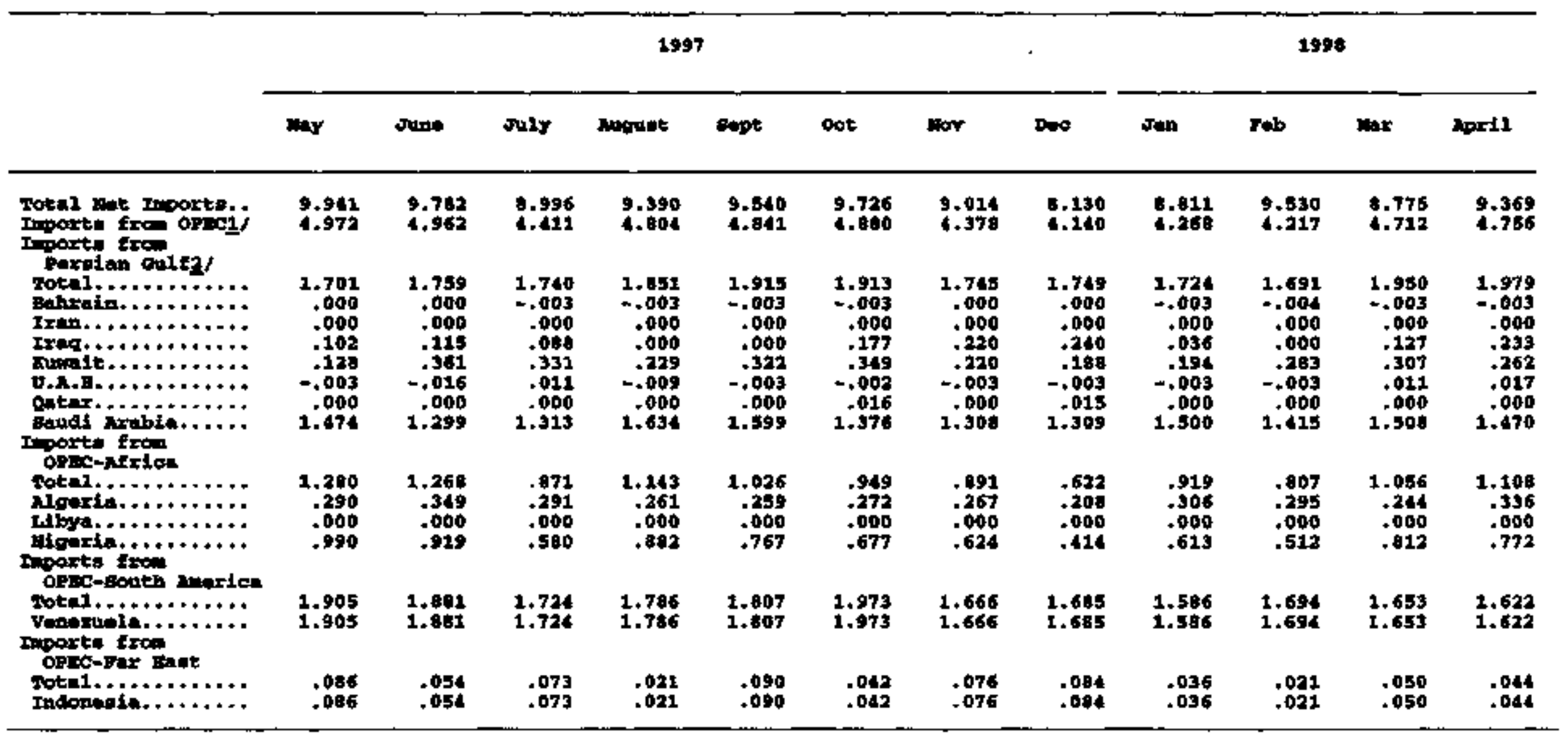

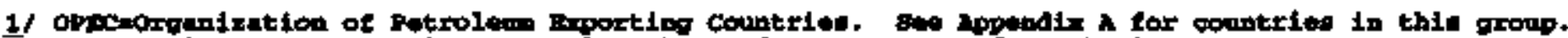

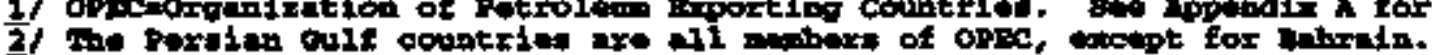

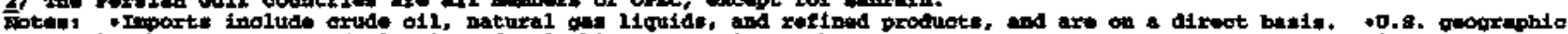

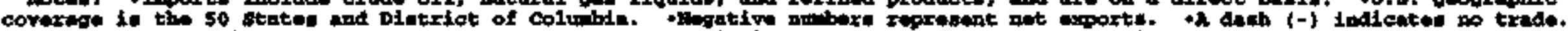

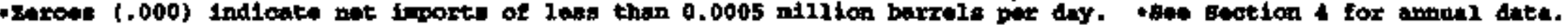

bonroed, sot the of section 3 . 


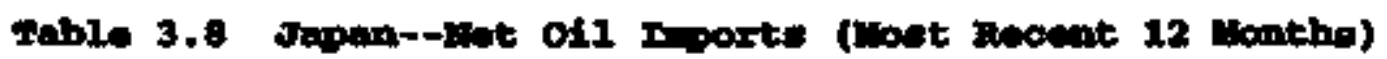

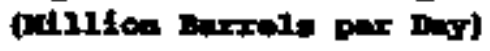

\begin{tabular}{|c|c|c|c|c|c|c|c|c|c|c|c|c|}
\hline & \multicolumn{8}{|c|}{1997} & \multicolumn{4}{|c|}{1998} \\
\hline Total wot Imocte. & $\begin{array}{l}5.218 \\
4.179\end{array}$ & 3.914 & $\begin{array}{l}3.586 \\
4.366\end{array}$ & 9.492 & $\begin{array}{l}5.007 \\
4.553\end{array}$ & $\begin{array}{l}5.755 \\
4.775\end{array}$ & $\begin{array}{l}5.912 \\
4.799\end{array}$ & $\begin{array}{l}6.069 \\
3.112\end{array}$ & $\begin{array}{l}5.721 \\
4.732\end{array}$ & $\begin{array}{l}5.557 \\
4.589\end{array}$ & $\begin{array}{l}3.760 \\
4.902\end{array}$ & $\begin{array}{l}5.203 \\
4.368\end{array}$ \\
\hline 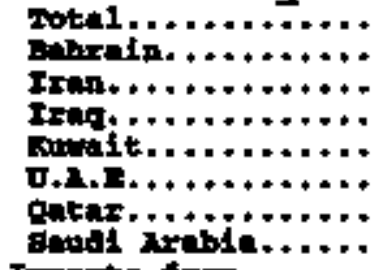 & $\begin{array}{r}3.816 \\
.031 \\
.009 \\
.059 \\
.464 \\
1.220 \\
.350 \\
1.284\end{array}$ & $\begin{array}{r}3.505 \\
.008 \\
.253 \\
.000 \\
.523 \\
1.160 \\
.291 \\
1.251\end{array}$ & $\begin{array}{r}3.909 \\
.017 \\
.466 \\
.000 \\
.473 \\
+.366 \\
.319 \\
1.267\end{array}$ & $\begin{array}{r}3.972 \\
.015 \\
.319 \\
.000 \\
.515 \\
1.420 \\
.420 \\
1.294\end{array}$ & $\begin{array}{r}4.147 \\
.037 \\
.464 \\
.000 \\
.970 \\
1.420 \\
. .316 \\
1.323\end{array}$ & $\begin{array}{r}1.518 \\
.014 \\
.401 \\
.000 \\
.542 \\
1.390 \\
.926 \\
1.745\end{array}$ & $\begin{array}{r}1.526 \\
.007 \\
.505 \\
.006 \\
.557 \\
1.506 \\
.406 \\
1.445\end{array}$ & $\begin{array}{r}4.705 \\
.031 \\
.546 \\
.000 \\
.630 \\
1.475 \\
.467 \\
1.556\end{array}$ & $\begin{array}{r}4.453 \\
.070 \\
.550 \\
.000 \\
.503 \\
1.545 \\
.469 \\
1.278\end{array}$ & $\begin{array}{r}1.312 \\
.017 \\
.453 \\
.000 \\
.570 \\
1.507 \\
. \$ 910\end{array}$ & $\begin{array}{r}4.545 \\
.000 \\
.592 \\
.000 \\
.611 \\
1.336 \\
1.571\end{array}$ & $\begin{array}{r}.098 \\
.000 \\
.282 \\
.000 \\
.432 \\
1.655 \\
1.293 \\
1.246\end{array}$ \\
\hline 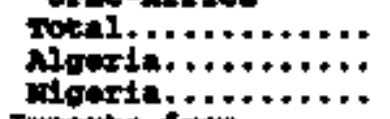 & $\begin{array}{l}.046 \\
.046 \\
.000\end{array}$ & $\begin{array}{r}.100 \\
.041 \\
.059\end{array}$ & $\begin{array}{l}.075 \\
.013 \\
.060\end{array}$ & $\begin{array}{l}.032 \\
.032 \\
.000\end{array}$ & $\begin{array}{l}.074 \\
.028 \\
.037\end{array}$ & $\begin{array}{l}.000 \\
.000 \\
.000\end{array}$ & $\begin{array}{l}.000 \\
.000 \\
.000\end{array}$ & $\begin{array}{l}.062 \\
.000 \\
.062\end{array}$ & $\begin{array}{l}.000 \\
.000 \\
.000\end{array}$ & $\begin{array}{l}.017 \\
.017 \\
.000\end{array}$ & $\begin{array}{l}.000 \\
.000 \\
.000\end{array}$ & $\begin{array}{l}.000 \\
.000 \\
.000\end{array}$ \\
\hline Total... & $\begin{array}{l}.000 \\
.000\end{array}$ & $\begin{array}{l}.000 \\
.000\end{array}$ & .000 & $\begin{array}{l}.000 \\
.000\end{array}$ & .000 & $\begin{array}{l}.000 \\
.000\end{array}$ & $\begin{array}{l}.000 \\
.000\end{array}$ & $\begin{array}{r}.003 \\
.001\end{array}$ & .000 & $\begin{array}{l}.000 \\
.000\end{array}$ & $\begin{array}{l}.000 \\
.000\end{array}$ & $\begin{array}{l}.000 \\
.000\end{array}$ \\
\hline$\forall i \ldots+\ldots \ldots+\ldots$ &. .344 & $\begin{array}{l}.300 \\
.380\end{array}$ &. .402 & .42 & $\begin{array}{r}.369 \\
.369\end{array}$ & .272 & $\begin{array}{r}.279 \\
.279\end{array}$ & $\begin{array}{r}.376 \\
.376\end{array}$ & $\begin{array}{r}.307 \\
.307\end{array}$ & $\begin{array}{r}.278 \\
.278\end{array}$ & .356 & $\begin{array}{r}.270 \\
.270\end{array}$ \\
\hline
\end{tabular}

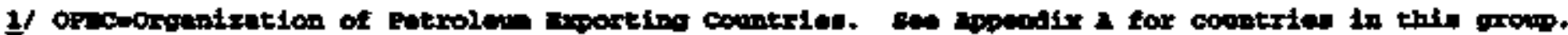

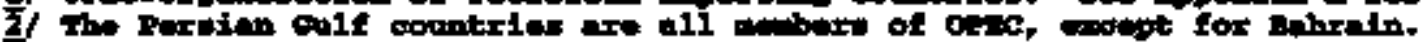

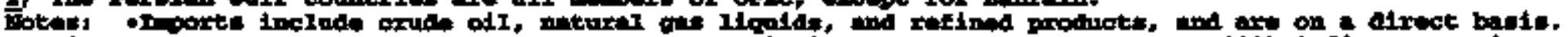

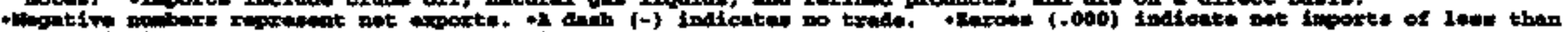

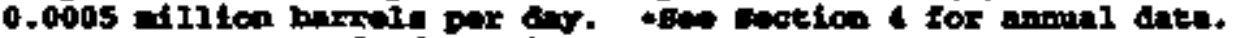

Bouroest Ben wa of Bection 3. 


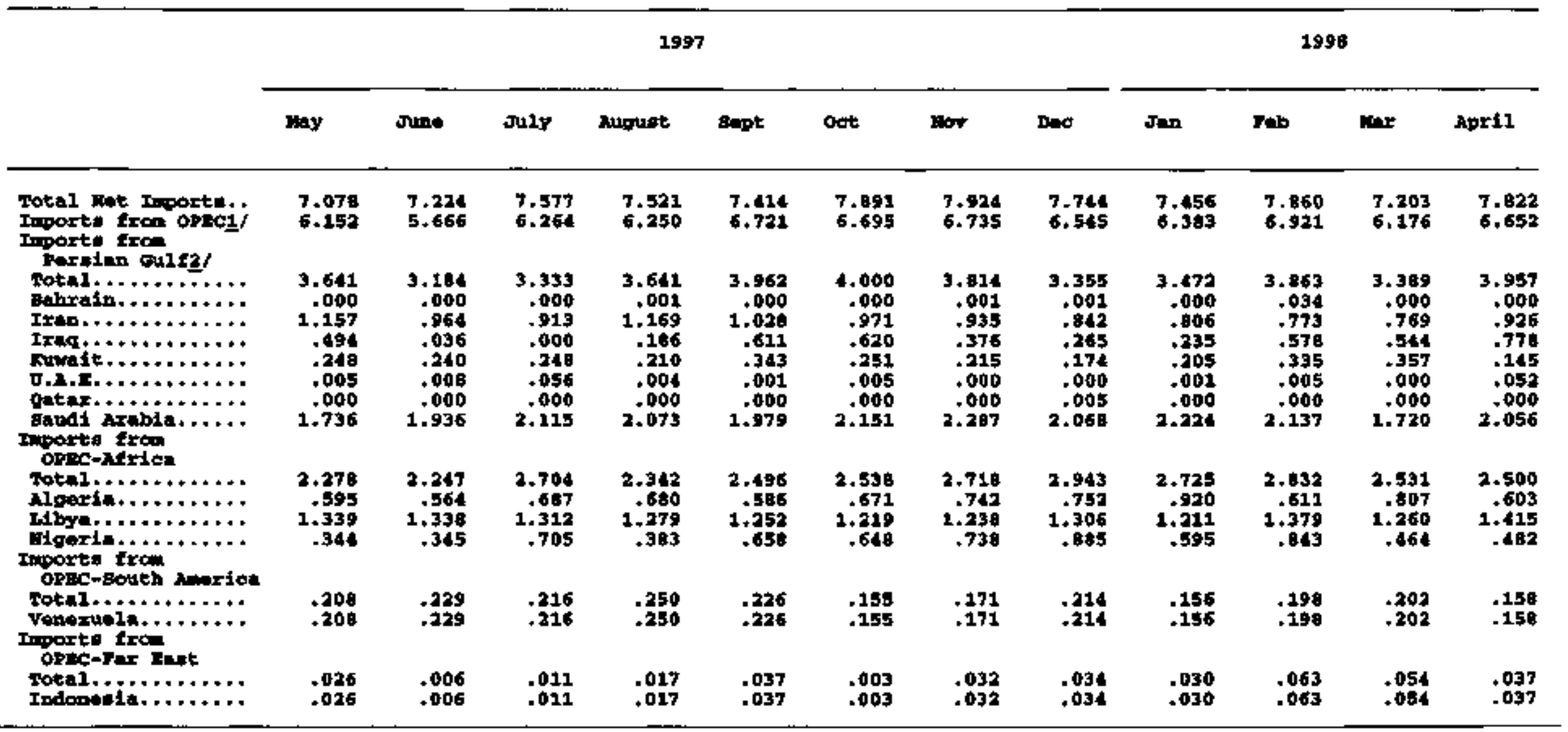

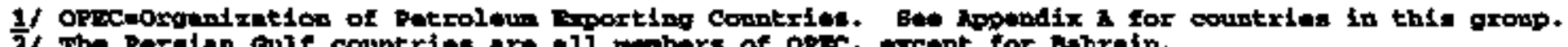

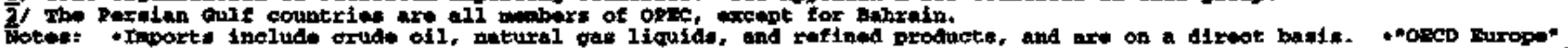

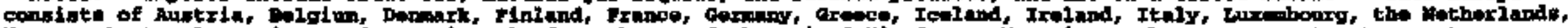

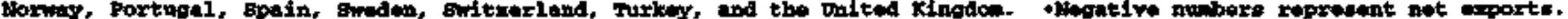

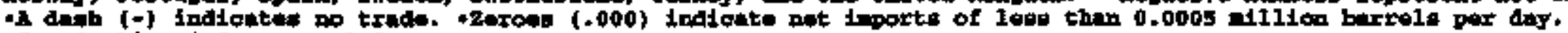

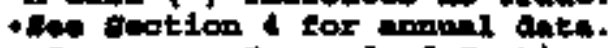

Boureast the end of enction 3 . 


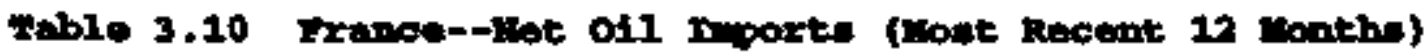

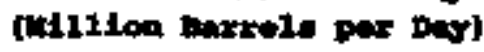

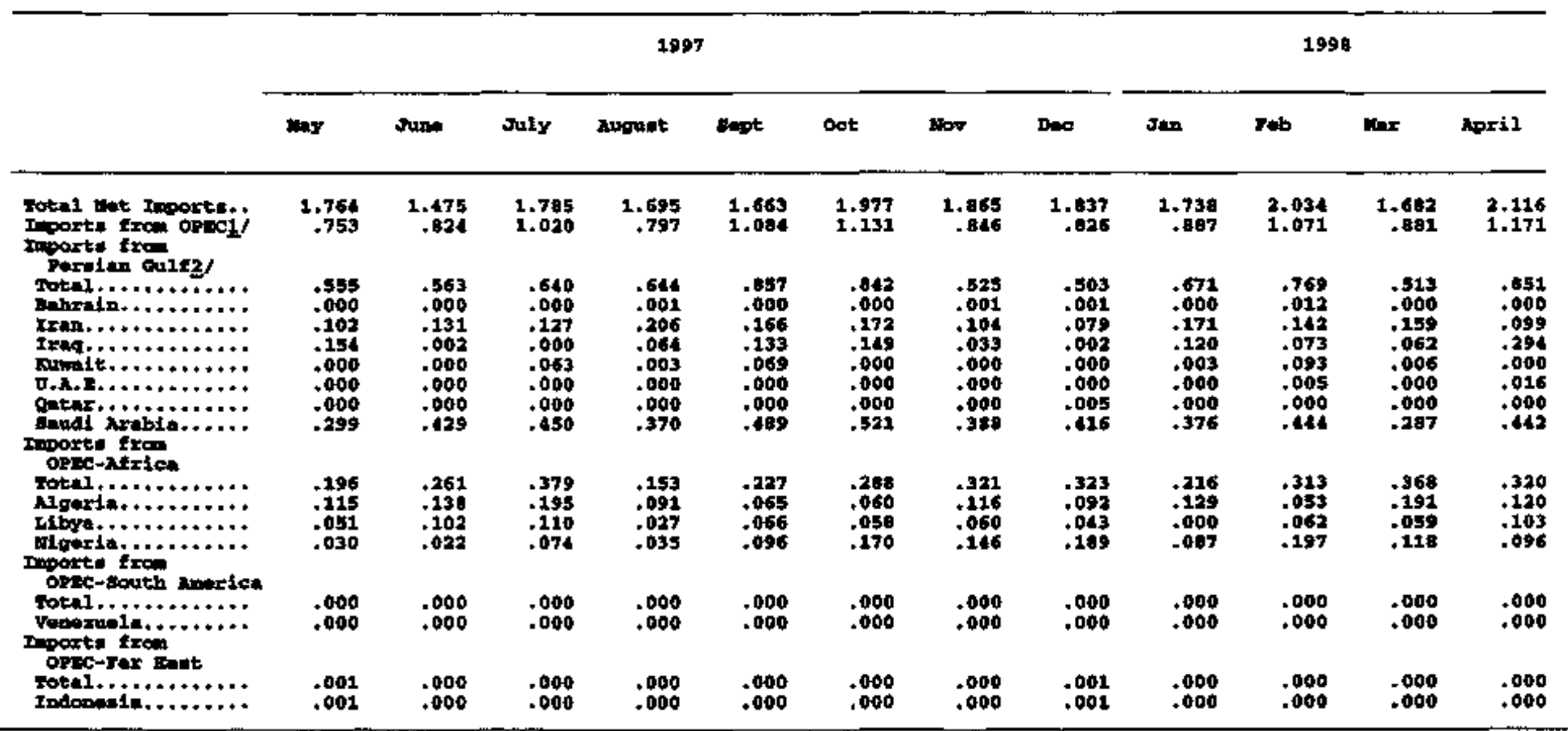

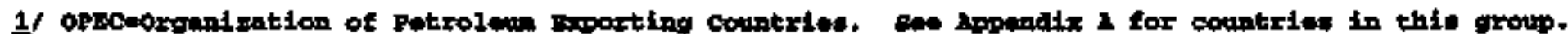

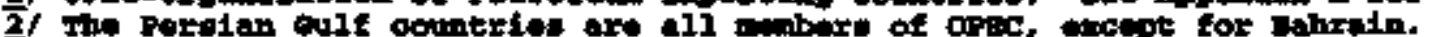

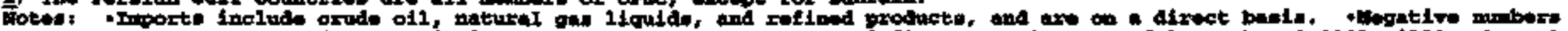

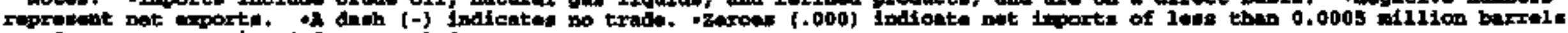

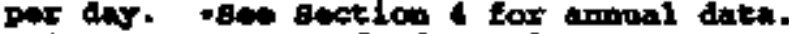

Bourcert seip and of soction 3 . 


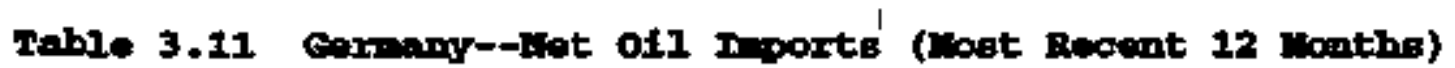

(kililon Bnrrele por Day)

\begin{tabular}{|c|c|c|c|c|c|c|c|c|c|c|c|c|}
\hline & \multicolumn{8}{|c|}{1997} & \multicolumn{4}{|c|}{1999} \\
\hline & $\operatorname{lin} y$ & Juso & soly & Mupoot & sopt & $\alpha=t$ & nov & Ders & $\operatorname{san}$ & rab & $\max$ & ansid \\
\hline 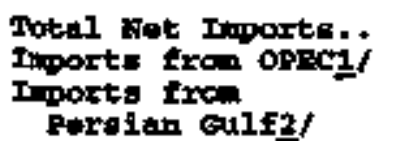 & $\begin{array}{r}2.954 \\
.683\end{array}$ & $\begin{array}{r}2.690 \\
.561\end{array}$ & $\begin{array}{r}3.123 \\
.657\end{array}$ & $\begin{array}{r}2.652 \\
.698\end{array}$ & $\begin{array}{r}2.766 \\
.636\end{array}$ & $\begin{array}{r}2.706 \\
.649\end{array}$ & $\begin{array}{r}2.030 \\
.647\end{array}$ & $\begin{array}{r}2.933 \\
.627\end{array}$ & $\begin{array}{r}2.635 \\
.601\end{array}$ & $\begin{array}{r}2.601 \\
.595\end{array}$ & $\begin{array}{r}2.938 \\
.571\end{array}$ & $\begin{array}{r}2.920 \\
.501\end{array}$ \\
\hline 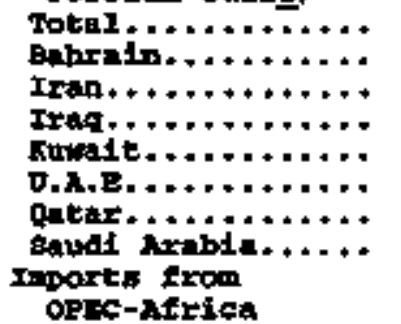 & $\begin{array}{l}.153 \\
.000 \\
.016 \\
.000 \\
.009 \\
.002 \\
.000 \\
.125\end{array}$ & $\begin{array}{l}.192 \\
.000 \\
.037 \\
.000 \\
.013 \\
.002 \\
.000 \\
.140\end{array}$ & $\begin{array}{l}.177 \\
.000 \\
.022 \\
.000 \\
.014 \\
.005 \\
.000 \\
.136\end{array}$ & $\begin{array}{l}.202 \\
.000 \\
.037 \\
.000 \\
.009 \\
.000 \\
.000 \\
.156\end{array}$ & $\begin{array}{l}.164 \\
.000 \\
.017 \\
.000 \\
.016 \\
.000 \\
.000 \\
.130\end{array}$ & $\begin{array}{l}.205 \\
.000 \\
.041 \\
.000 \\
.012 \\
.000 \\
.000 \\
.152\end{array}$ & $\begin{array}{l}.155 \\
.000 \\
.019 \\
.009 \\
.014 \\
.000 \\
.000 \\
.113\end{array}$ & $\begin{array}{l}.131 \\
.000 \\
.010 \\
.000 \\
.013 \\
.000 \\
.000 \\
.108\end{array}$ & $\begin{array}{l}.101 \\
.000 \\
.004 \\
.000 \\
.004 \\
.000 \\
.000 \\
.093\end{array}$ & $\begin{array}{l}.124 \\
.000 \\
.000 \\
.000 \\
.020 \\
.000 \\
.000 \\
.104\end{array}$ & $\begin{array}{l}.115 \\
.000 \\
.027 \\
.000 \\
.016 \\
.000 \\
.000 \\
.072\end{array}$ & $\begin{array}{l}.178 \\
.000 \\
.014 \\
.034 \\
.021 \\
.000 \\
.000 \\
.109\end{array}$ \\
\hline 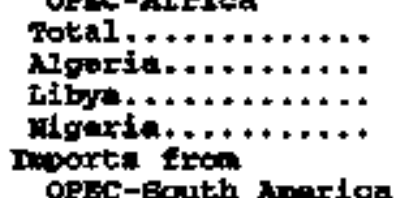 & $\begin{array}{l}.070 \\
.054 \\
.318 \\
.097\end{array}$ & $\begin{array}{l}.352 \\
.070 \\
.261 \\
.021\end{array}$ & $\begin{array}{l}.378 \\
.061 \\
.253 \\
.066\end{array}$ & $\begin{array}{l}.659 \\
.092 \\
.273 \\
.095\end{array}$ & $\begin{array}{l}.131 \\
.091 \\
.267 \\
.073\end{array}$ & $\begin{array}{l}.401 \\
.106 \\
.215 \\
.040\end{array}$ & $\begin{array}{l}.449 \\
.087 \\
.261 \\
.102\end{array}$ & $\begin{array}{l}.146 \\
.146 \\
.172 \\
.128\end{array}$ & $\begin{array}{l}.490 \\
.225 \\
.241 \\
.124\end{array}$ & $\begin{array}{l}.439 \\
.091 \\
.288 \\
.059\end{array}$ & $\begin{array}{l}.410 \\
.150 \\
.252 \\
.008\end{array}$ & $\begin{array}{l}.368 \\
.050 \\
.275 \\
.043\end{array}$ \\
\hline 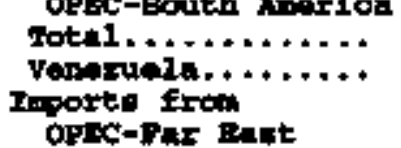 & $\begin{array}{l}.059 \\
.059\end{array}$ & $\begin{array}{l}.037 \\
.037\end{array}$ & $\begin{array}{r}.101 \\
.101\end{array}$ & $\begin{array}{l}.036 \\
.036\end{array}$ & .041 & $\begin{array}{r}.043 \\
.043\end{array}$ & .043 & .051 & $\begin{array}{l}.006 \\
.006\end{array}$ & $\begin{array}{l}.032 \\
.032\end{array}$ & .046 & $\begin{array}{r}.055 \\
.055\end{array}$ \\
\hline Total $19 . \ldots \ldots \ldots$ & $\begin{array}{l}.001 \\
.001\end{array}$ & $\begin{array}{l}.000 \\
.000\end{array}$ & $\begin{array}{l}.001 \\
.001\end{array}$ & .000 & $\begin{array}{l}.000 \\
.000\end{array}$ & $\begin{array}{l}.000 \\
.000\end{array}$ & $\begin{array}{l}.000 \\
.000\end{array}$ & $\begin{array}{l}.000 \\
.000\end{array}$ & .004 & $\begin{array}{l}.000 \\
.000\end{array}$ & .000 & .000 \\
\hline
\end{tabular}

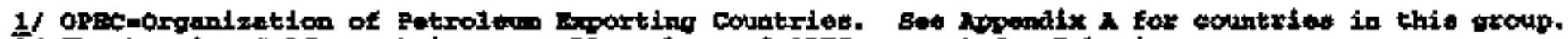

2/ The Poraisn oulf countries are all menbert of OpFC, excopt for Buhrein.

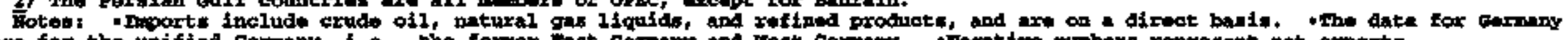

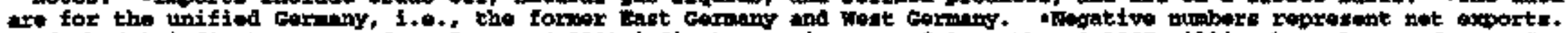

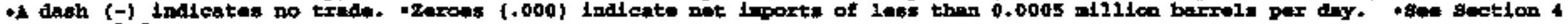
Eor anmual date.

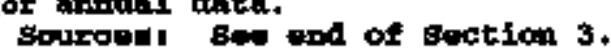




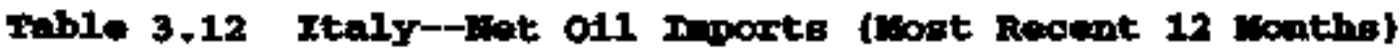

(Milliod Barroln par Dar)

1997

1998

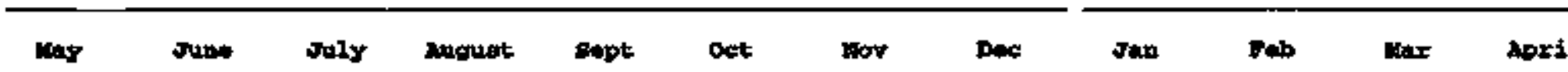

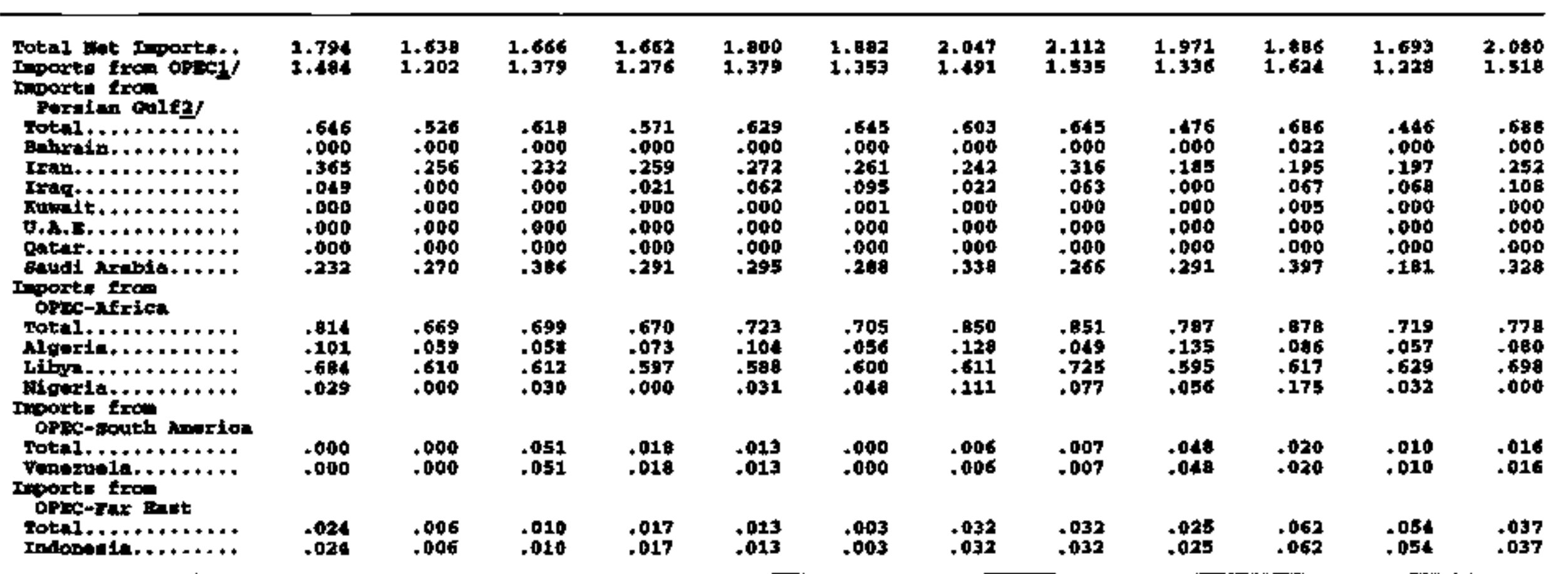

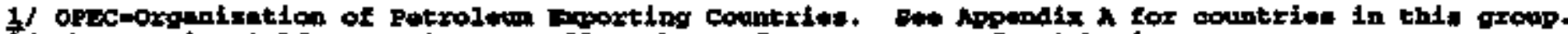

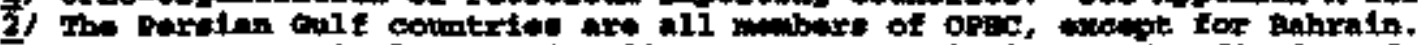

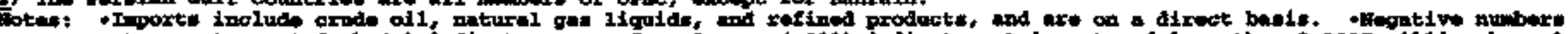

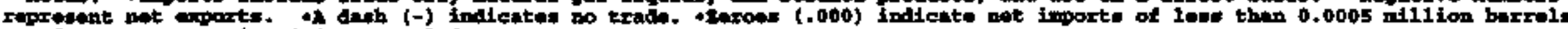

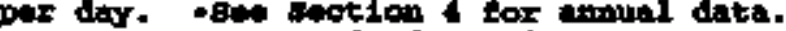

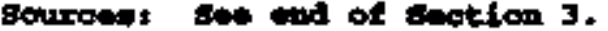




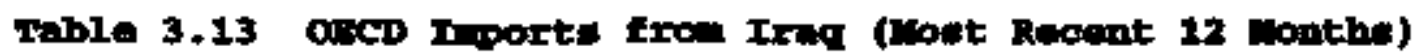

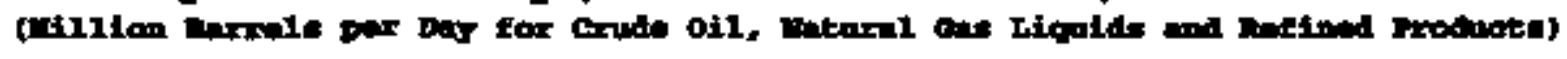

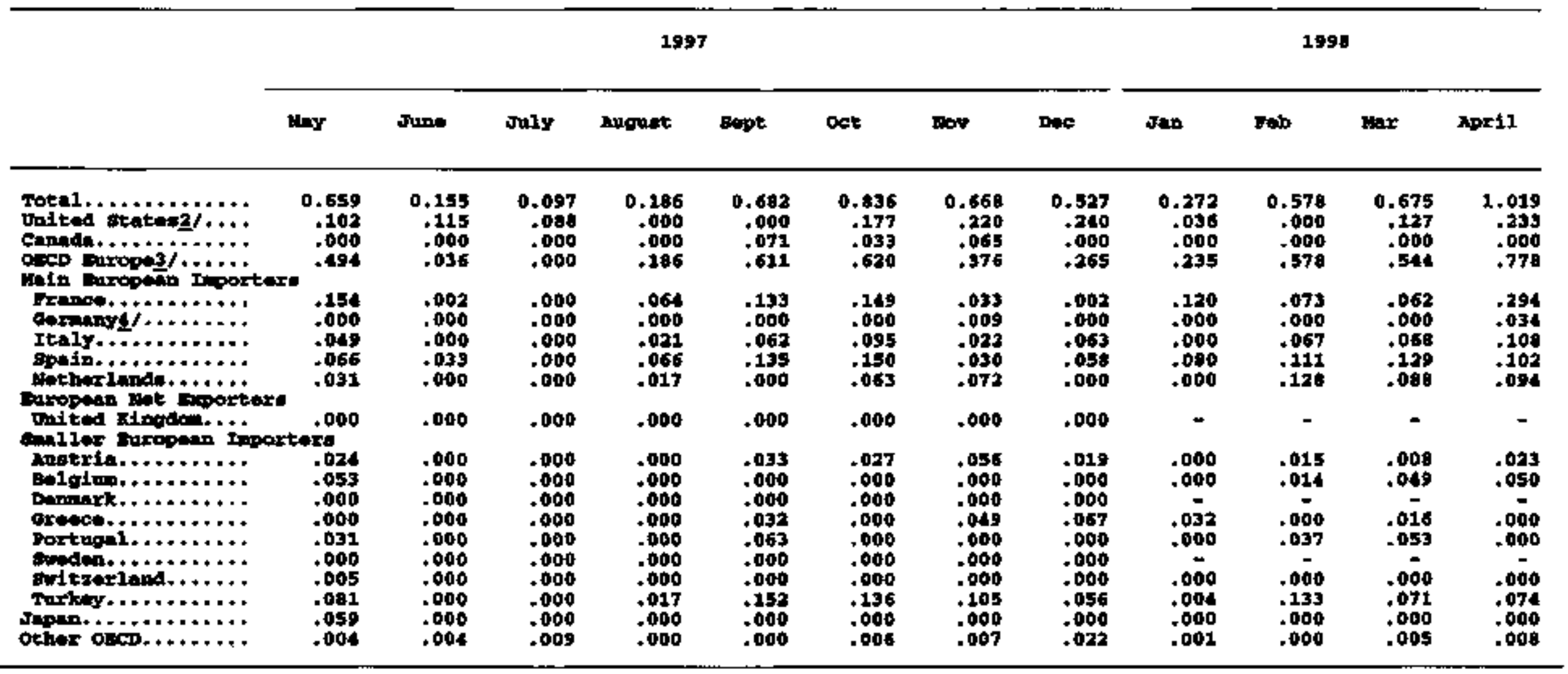

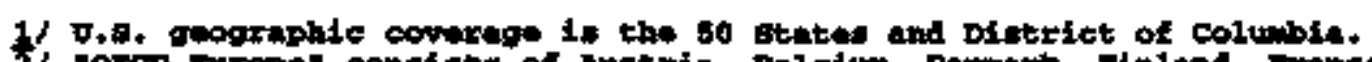

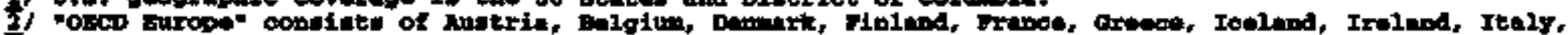

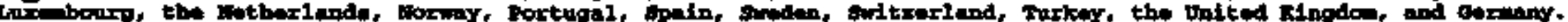

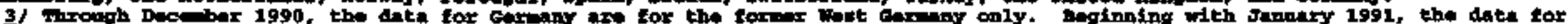

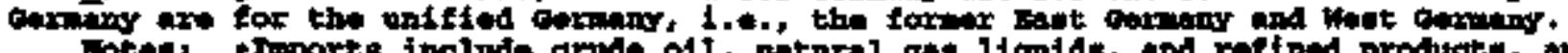

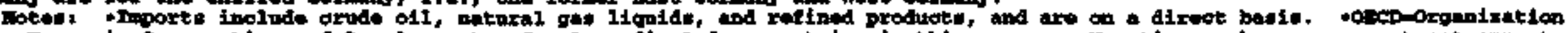

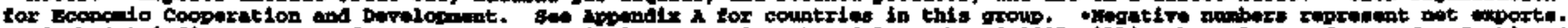

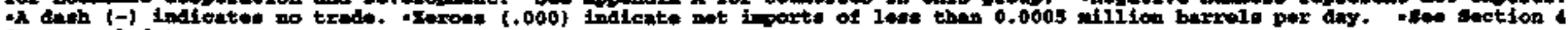
for amman daten.

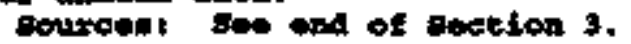




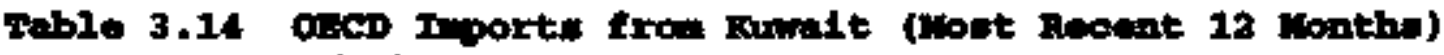

(wilico Barrelle per Day)

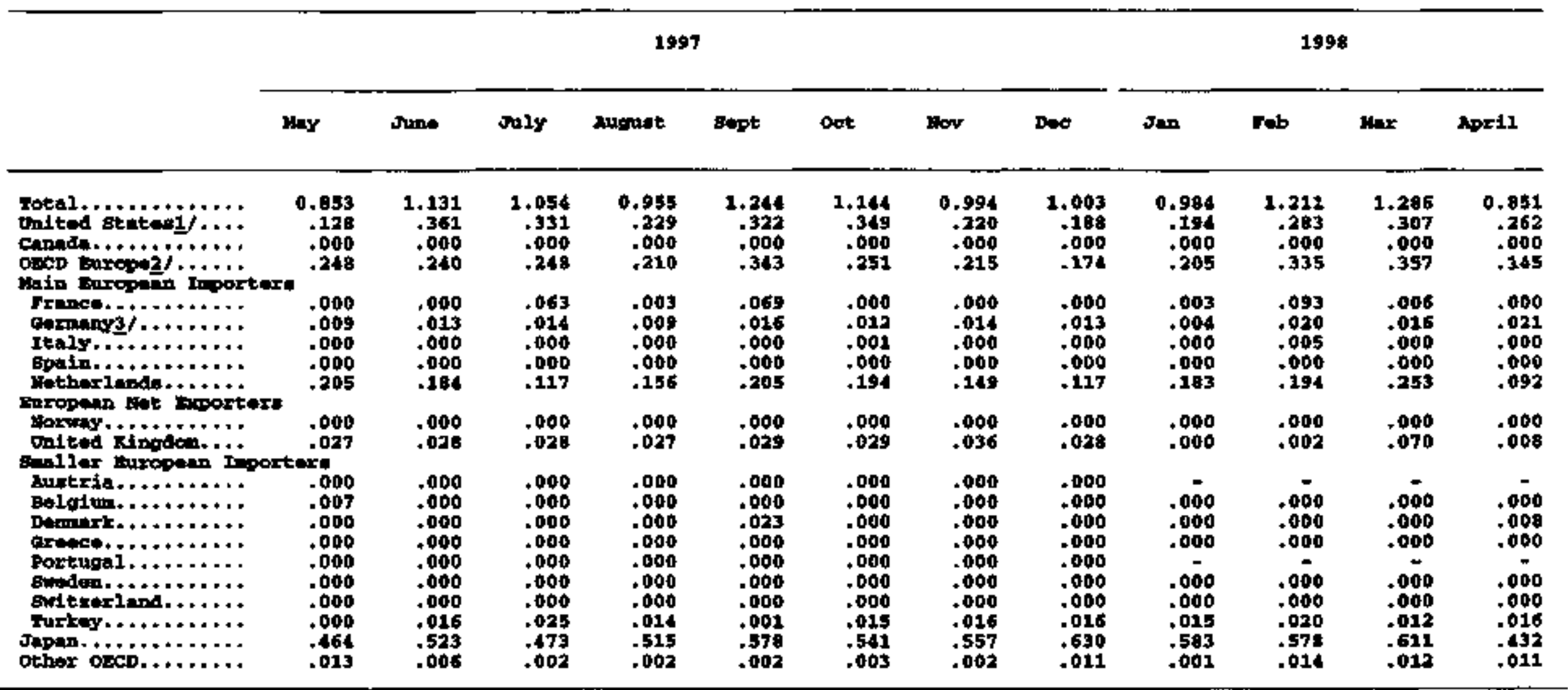

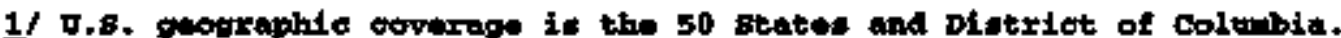

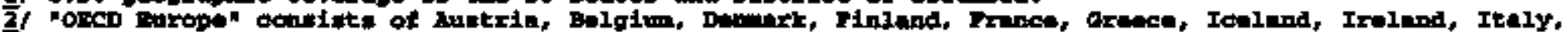

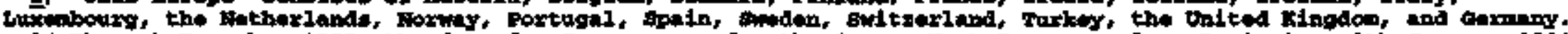

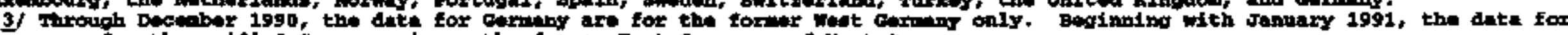

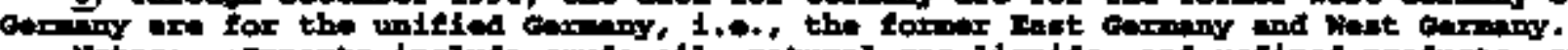

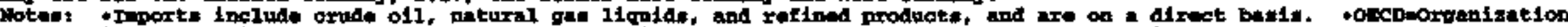

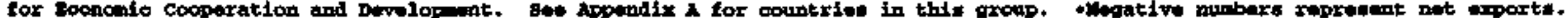

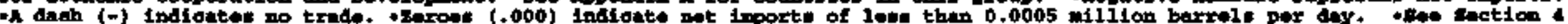

$\operatorname{los}$ antuid dete.

Boturoen: wase and of Bention 3. 


\section{Sources for Section 3 (International Oil Imports)}

\section{Oit Imports}

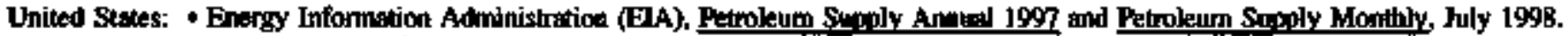

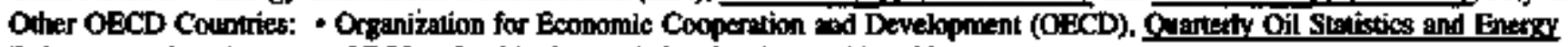

Batupces various isspes. - OECD, Monthly Oil Stutstics dala base, 199]-1998. 
4. Hlstorical Oil Data Series 
Table 4.1e Norld Cxude oil Pxoduction (Imcluding Ieare Condeneate), 1970-1997

(moonnod Barrele jer Day)

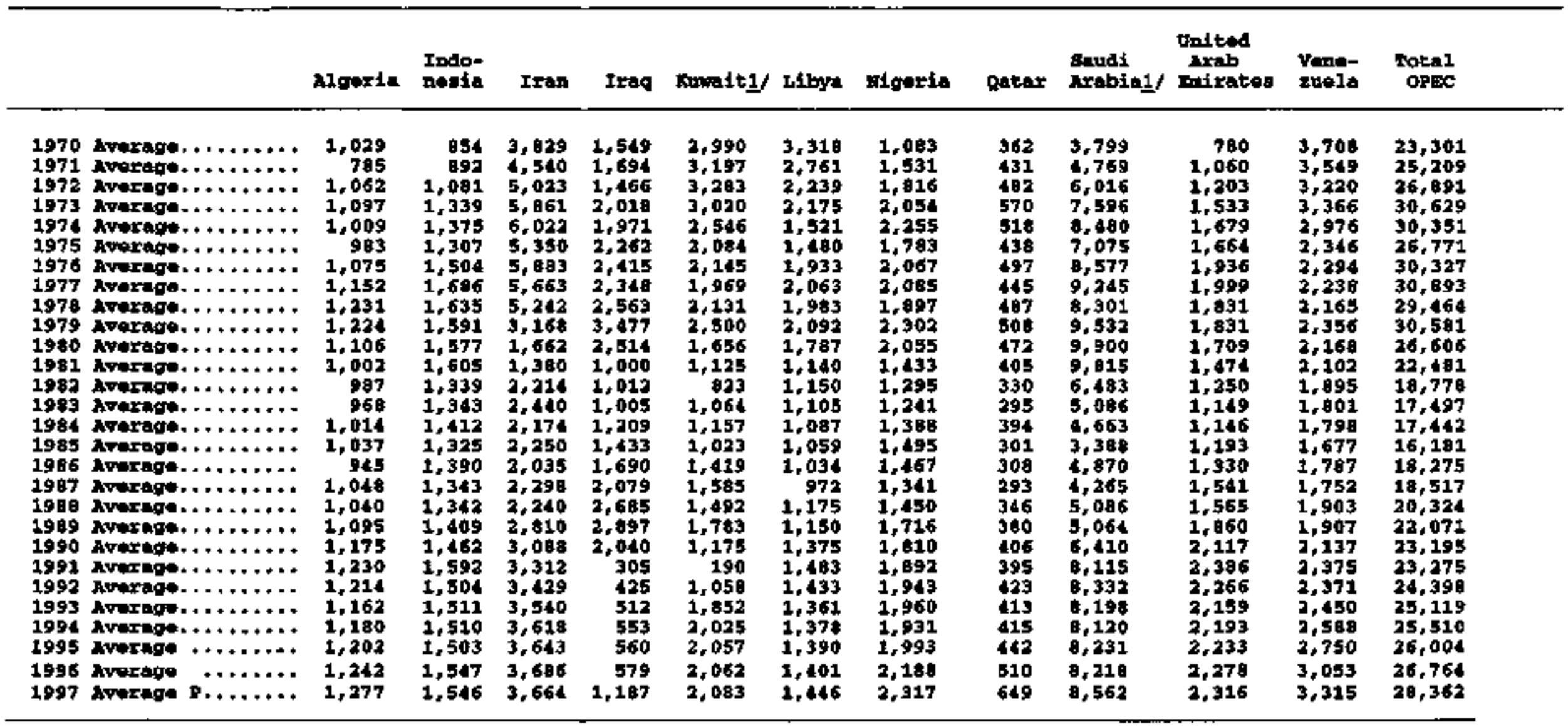

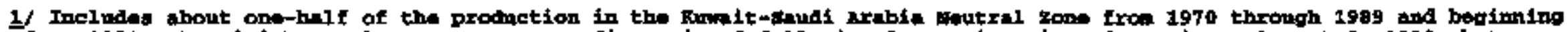

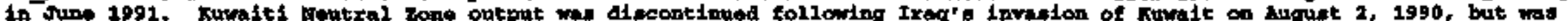

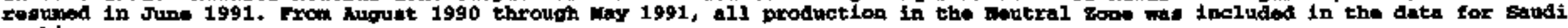
arabia.

Peprelinimary.

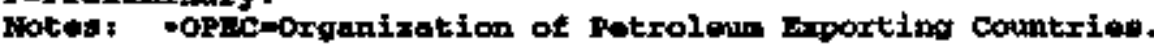

sourtev I tere ond of seation 4 


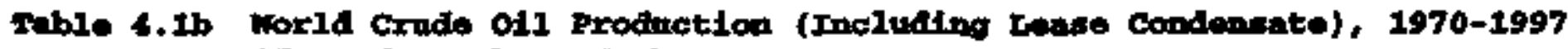

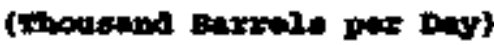

\begin{tabular}{|c|c|c|c|c|c|c|c|c|c|c|c|c|}
\hline & & Lorway & $\begin{array}{l}\text { Cont tod } \\
\text { Ktnodor }\end{array}$ & $\begin{array}{l}\text { Wotth } \\
\text { sent/ }\end{array}$ & ansola & $\begin{array}{c}\text { neres- } \\
\text { tInth }\end{array}$ & metra- & Brazil & cuntala & Chim & colopbla & Ictudiox \\
\hline $\begin{array}{l}1970 \\
1971 \\
1972 \\
1973 \\
1974 \\
1975 \\
1976 \\
1977 \\
1978 \\
1979 \\
1980 \\
1981 \\
1982 \\
1983 \\
1984 \\
1985 \\
1986 \\
1987 \\
1988 \\
1989 \\
1990 \\
1991 \\
1992 \\
1993 \\
1994 \\
1995 \\
1996 \\
1997\end{array}$ & 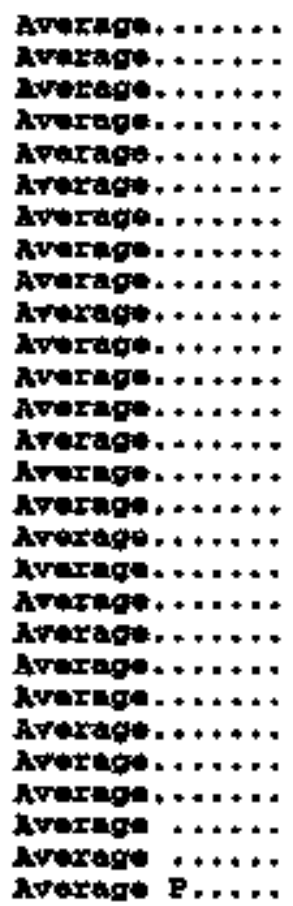 & $\begin{array}{r}0 \\
33 \\
31 \\
35 \\
199 \\
279 \\
280 \\
356 \\
493 \\
528 \\
501 \\
500 \\
614 \\
697 \\
798 \\
670 \\
1,022 \\
1,158 \\
1,554 \\
1,704 \\
1,890 \\
2,229 \\
2,350 \\
2,521 \\
2,768 \\
3,104 \\
3,153\end{array}$ & $\begin{array}{r}2 \\
2 \\
3 \\
2 \\
12 \\
245 \\
768 \\
1,082 \\
1,568 \\
1,622 \\
1,811 \\
2,065 \\
2,291 \\
2,480 \\
2,530 \\
2,539 \\
2,406 \\
2,232 \\
1,802 \\
1,820 \\
1,797 \\
1,825 \\
1,915 \\
2,375 \\
2,409 \\
3,560 \\
2,517\end{array}$ & $\begin{array}{r}6 \\
35 \\
36 \\
30 \\
202 \\
525 \\
1,055 \\
1,446 \\
1,976 \\
2,151 \\
2,322 \\
2,617 \\
2,965 \\
3,250 \\
3,417 \\
3,547 \\
3,584 \\
3,540 \\
3,515 \\
3,667 \\
3,811 \\
4,283 \\
4,603 \\
5,058 \\
5,400 \\
5,622 \\
5,843\end{array}$ & $\begin{array}{r}112 \\
93 \\
140 \\
162 \\
169 \\
165 \\
100 \\
194 \\
131 \\
147 \\
150 \\
130 \\
122 \\
177 \\
209 \\
231 \\
282 \\
960 \\
452 \\
455 \\
475 \\
500 \\
526 \\
509 \\
536 \\
646 \\
709 \\
720\end{array}$ & $\begin{array}{l}393 \\
423 \\
433 \\
420 \\
414 \\
395 \\
398 \\
491 \\
453 \\
473 \\
491 \\
496 \\
491 \\
491 \\
470 \\
460 \\
434 \\
478 \\
449 \\
460 \\
463 \\
485 \\
553 \\
594 \\
650 \\
715 \\
756 \\
835\end{array}$ & $\begin{array}{l}178 \\
309 \\
327 \\
369 \\
388 \\
409 \\
426 \\
431 \\
432 \\
441 \\
380 \\
394 \\
370 \\
416 \\
492 \\
575 \\
520 \\
547 \\
536 \\
490 \\
575 \\
545 \\
535 \\
503 \\
536 \\
562 \\
570 \\
586\end{array}$ & $\begin{array}{l}167 \\
174 \\
167 \\
169 \\
176 \\
177 \\
167 \\
161 \\
160 \\
166 \\
182 \\
213 \\
260 \\
339 \\
475 \\
564 \\
572 \\
566 \\
854 \\
596 \\
631 \\
630 \\
626 \\
643 \\
671 \\
695 \\
795 \\
\$ 93\end{array}$ & $\begin{array}{l}1,263 \\
1,348 \\
1,532 \\
1,798 \\
1,591 \\
1,430 \\
1,314 \\
1,321 \\
1,316 \\
1,500 \\
1,435 \\
1,285 \\
1,271 \\
1,356 \\
1,438 \\
1,471 \\
1,474 \\
1,535 \\
1,616 \\
1,560 \\
1,553 \\
1,548 \\
1,605 \\
1,679 \\
1,746 \\
1,605 \\
1,637 \\
1,093\end{array}$ & $\begin{array}{r}600 \\
780 \\
900 \\
1,090 \\
1,315 \\
1,490 \\
1,670 \\
1,874 \\
2,092 \\
2,125 \\
2,114 \\
2,012 \\
2,045 \\
2,120 \\
3,296 \\
2,509 \\
2,620 \\
2,690 \\
2,790 \\
2,757 \\
2,774 \\
3,835 \\
2,645 \\
3,690 \\
2,939 \\
2,990 \\
3,131 \\
3,200\end{array}$ & $\begin{array}{l}219 \\
214 \\
196 \\
184 \\
168 \\
157 \\
146 \\
136 \\
131 \\
124 \\
126 \\
125 \\
141 \\
152 \\
168 \\
176 \\
305 \\
395 \\
378 \\
403 \\
440 \\
119 \\
435 \\
456 \\
450 \\
585 \\
623 \\
662\end{array}$ & $\begin{array}{r}4 \\
78 \\
209 \\
177 \\
161 \\
109 \\
183 \\
202 \\
214 \\
204 \\
311 \\
211 \\
237 \\
258 \\
281 \\
293 \\
174 \\
302 \\
279 \\
205 \\
299 \\
321 \\
344 \\
365 \\
392 \\
396 \\
391\end{array}$ \\
\hline
\end{tabular}

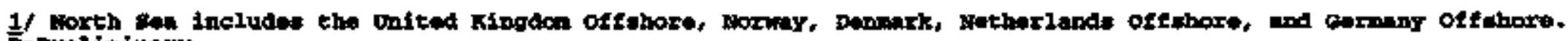
Fepratibinary.

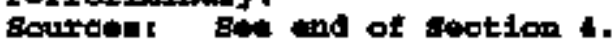


Tubl 4.1c World Crude ol1 Production (Incloding Ioase condonnate), 1970-1997

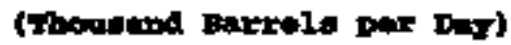

\begin{tabular}{|c|c|c|c|c|c|c|c|c|c|c|c|c|c|}
\hline & & Beppt & Aabon & Indite & valeyoin & tortoo & סותם & thonete & $\begin{array}{l}\text { Fon } \\
\text { t.B+B.R. }\end{array}$ & Birxte & $\begin{array}{l}\text { Dnited } \\
\text { stater }\end{array}$ & Othax1/ & Forld \\
\hline $\begin{array}{l}1970 \\
1971 \\
1972 \\
1973 \\
1974 \\
1975 \\
1976 \\
1977 \\
1978 \\
1979 \\
1980 \\
1981 \\
1992 \\
1903 \\
1904 \\
1995 \\
1986 \\
1987 \\
1980 \\
1989 \\
1990 \\
1991 \\
1992 \\
1993 \\
1994 \\
1993 \\
1996 \\
1997\end{array}$ & 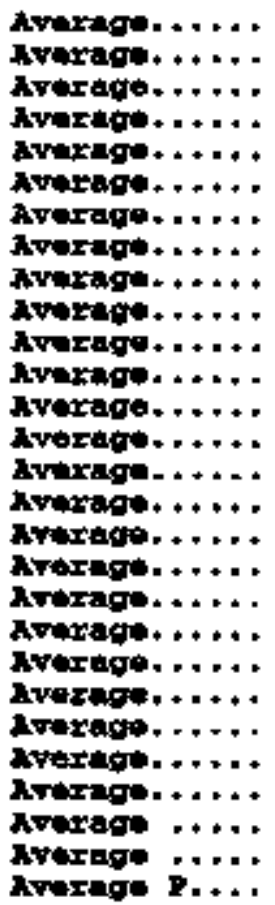 & $\begin{array}{l}326 \\
293 \\
231 \\
165 \\
180 \\
235 \\
330 \\
415 \\
495 \\
525 \\
595 \\
598 \\
670 \\
727 \\
822 \\
887 \\
613 \\
896 \\
048 \\
665 \\
873 \\
974 \\
981 \\
690 \\
696 \\
920 \\
922 \\
874\end{array}$ & $\begin{array}{l}109 \\
115 \\
125 \\
150 \\
202 \\
223 \\
229 \\
222 \\
209 \\
203 \\
175 \\
151 \\
156 \\
157 \\
157 \\
172 \\
166 \\
155 \\
159 \\
209 \\
270 \\
394 \\
298 \\
313 \\
329 \\
365 \\
369 \\
368\end{array}$ & $\begin{array}{l}144 \\
143 \\
156 \\
147 \\
156 \\
165 \\
175 \\
199 \\
236 \\
245 \\
102 \\
325 \\
390 \\
490 \\
515 \\
620 \\
630 \\
609 \\
635 \\
700 \\
660 \\
615 \\
561 \\
534 \\
590 \\
703 \\
651 \\
675\end{array}$ & $\begin{array}{l}18 \\
69 \\
93 \\
91 \\
61 \\
98 \\
165 \\
184 \\
217 \\
283 \\
283 \\
264 \\
306 \\
365 \\
440 \\
440 \\
504 \\
497 \\
540 \\
585 \\
619 \\
646 \\
653 \\
640 \\
645 \\
682 \\
695 \\
746\end{array}$ & $\begin{array}{r}487 \\
486 \\
505 \\
465 \\
571 \\
705 \\
831 \\
981 \\
1,209 \\
1,461 \\
1,936 \\
2,313 \\
2,748 \\
2,689 \\
2,780 \\
2,745 \\
2,435 \\
2,548 \\
2,512 \\
3,520 \\
2,553 \\
2,690 \\
2,669 \\
2,673 \\
2,685 \\
2,616 \\
2,555 \\
3,023\end{array}$ & $\begin{array}{l}333 \\
294 \\
282 \\
293 \\
290 \\
343 \\
366 \\
341 \\
315 \\
295 \\
292 \\
319 \\
324 \\
375 \\
414 \\
498 \\
560 \\
582 \\
617 \\
641 \\
685 \\
700 \\
740 \\
776 \\
810 \\
851 \\
683 \\
887\end{array}$ & 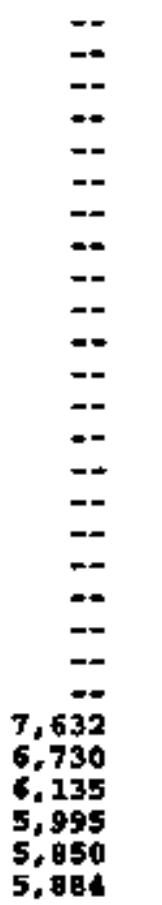 & 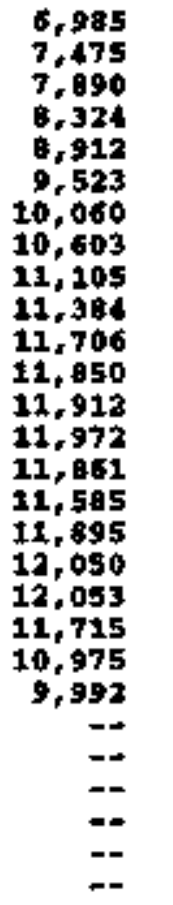 & $\begin{array}{r}60 \\
100 \\
124 \\
105 \\
124 \\
170 \\
175 \\
189 \\
170 \\
166 \\
164 \\
166 \\
160 \\
167 \\
170 \\
178 \\
194 \\
290 \\
265 \\
340 \\
388 \\
492 \\
461 \\
554 \\
560 \\
575 \\
604 \\
561\end{array}$ & $\begin{array}{l}9,637 \\
9,463 \\
9,441 \\
9,209 \\
8,774 \\
8,375 \\
9,132 \\
1,245 \\
6,707 \\
8,552 \\
8,597 \\
6,572 \\
8,649 \\
8,688 \\
8,879 \\
8,971 \\
9,680 \\
9,349 \\
0,140 \\
7,613 \\
7,355 \\
7,417 \\
7,171 \\
6,847 \\
6,662 \\
6,560 \\
6,465 \\
6,452\end{array}$ & $\begin{array}{l}2,529 \\
1,518 \\
1,592 \\
1,667 \\
7,711 \\
1,636 \\
1,620 \\
1,655 \\
1,706 \\
1,621 \\
1,042 \\
1,859 \\
1,892 \\
1,946 \\
2,015 \\
2,124 \\
2,166 \\
2,120 \\
2,235 \\
2,249 \\
2,253 \\
2,274 \\
3,226 \\
3,280 \\
3,382 \\
3,410 \\
3,509 \\
3,706\end{array}$ & $\begin{array}{l}45,896 \\
48,518 \\
51,138 \\
55,679 \\
55,716 \\
52,828 \\
57,344 \\
59,707 \\
60,158 \\
62,674 \\
59,600 \\
56,076 \\
53,461 \\
53,256 \\
54,489 \\
53,992 \\
56,227 \\
96,666 \\
58,737 \\
59,863 \\
60,566 \\
60,207 \\
60,212 \\
60,238 \\
60,992 \\
62,331 \\
64,054 \\
66,317\end{array}$ \\
\hline
\end{tabular}

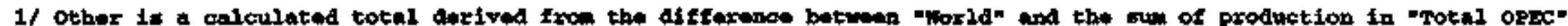

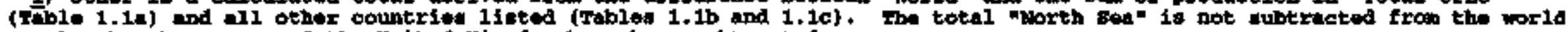
totel, thoust Foxway and the tontted Xingden bare been aubtracted.

- = Wot apolicable. PeProltminary.

sources Ber and of suction 4. 
rable 4,2 opre crude oil Production (Frluding Coudnates, 1980-1997

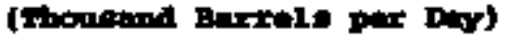

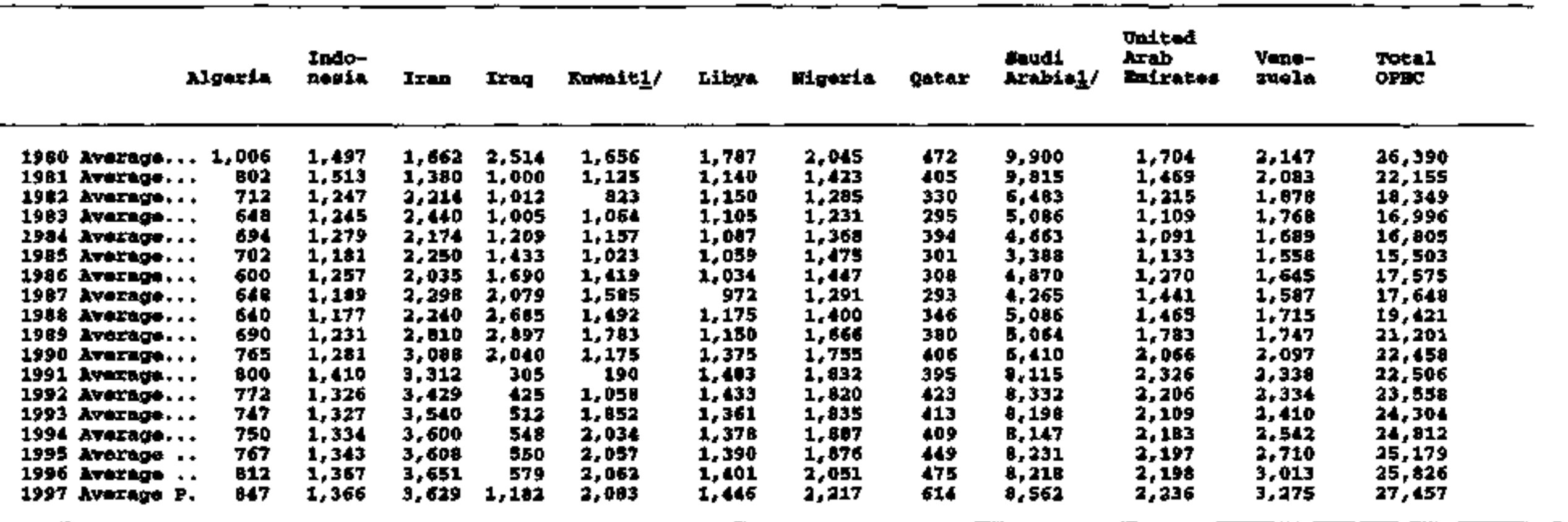

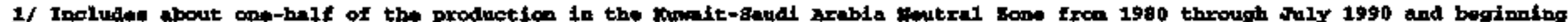

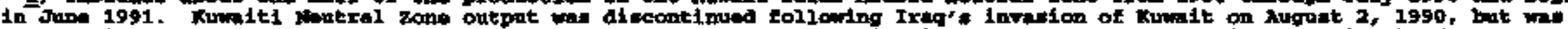

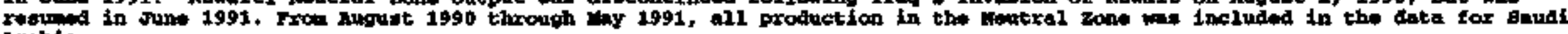
Mrobsat.

PeFreliningr.

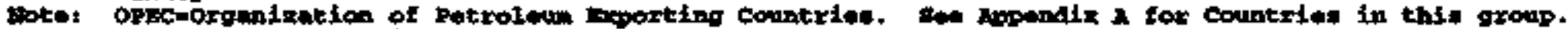

Schiroter Eov and of gection a. 
Table 4.3 World katural Gas plant Itquid: production, 1970-1997 (rhougand larrele per pery)

\begin{tabular}{|c|c|c|c|c|c|c|c|c|c|c|c|c|c|}
\hline & & & raxta & chrada & Woxtco & $\begin{array}{l}\text { gatods } \\
\text { arablat }\end{array}$ & Ruabla & $\begin{array}{l}\text { Pownity } \\
\text { Desif }\end{array}$ & $\begin{array}{l}\text { Onited } \\
\text { stutes } 1 \text { / }\end{array}$ & Pargitun & appecs/ & or:ay & Forld \\
\hline $\begin{array}{l}1970 \\
1971 \\
1972 \\
1973 \\
1974 \\
1975 \\
1976 \\
1977 \\
1970 \\
1979 \\
1980 \\
1981 \\
1982 \\
1983 \\
1984 \\
1985 \\
1986 \\
1987 \\
1988 \\
1989 \\
1990 \\
1991 \\
1992 \\
1993 \\
1994 \\
1995 \\
1996 \\
1997\end{array}$ & 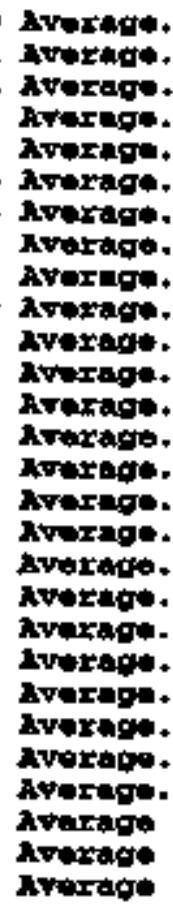 & $\begin{array}{l}\ldots \ldots \\
\ldots \ldots \\
\ldots \ldots \\
\ldots \ldots \\
\ldots \ldots \\
\ldots \ldots \\
\ldots \ldots \\
\ldots \ldots \\
\ldots \ldots \\
\ldots \ldots \\
\ldots \ldots \\
\ldots \ldots \\
\ldots \ldots \\
\ldots \ldots \\
\ldots \ldots \\
\ldots \ldots \\
\ldots \ldots \\
\ldots \ldots\end{array}$ & $\begin{array}{r}3 \\
4 \\
9 \\
12 \\
20 \\
24 \\
19 \\
25 \\
30 \\
36 \\
49 \\
50 \\
56 \\
105 \\
120 \\
120 \\
140 \\
120 \\
130 \\
130 \\
140 \\
140 \\
145 \\
140 \\
145 \\
150 \\
145\end{array}$ & $\begin{array}{l}200 \\
231 \\
293 \\
314 \\
314 \\
309 \\
289 \\
290 \\
281 \\
331 \\
331 \\
330 \\
310 \\
309 \\
336 \\
337 \\
326 \\
367 \\
381 \\
410 \\
426 \\
431 \\
460 \\
506 \\
529 \\
581 \\
596 \\
631\end{array}$ & $\begin{array}{l}58 \\
59 \\
61 \\
75 \\
90 \\
80 \\
95 \\
105 \\
115 \\
150 \\
193 \\
241 \\
255 \\
265 \\
257 \\
271 \\
352 \\
338 \\
370 \\
384 \\
420 \\
457 \\
454 \\
459 \\
451 \\
447 \\
423 \\
386\end{array}$ & $\begin{array}{r}50 \\
50 \\
55 \\
90 \\
130 \\
140 \\
185 \\
215 \\
250 \\
303 \\
369 \\
439 \\
430 \\
330 \\
355 \\
375 \\
385 \\
418 \\
499 \\
503 \\
620 \\
690 \\
713 \\
704 \\
698 \\
701 \\
697 \\
723\end{array}$ & 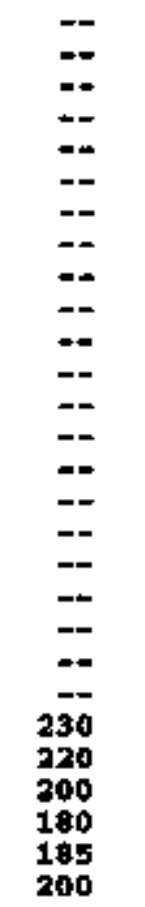 & $\begin{array}{l}120 \\
135 \\
155 \\
170 \\
190 \\
205 \\
220 \\
235 \\
255 \\
270 \\
285 \\
300 \\
315 \\
330 \\
340 \\
350 \\
440 \\
430 \\
450 \\
425 \\
425 \\
420 \\
-= \\
-2 \\
-= \\
-2 \\
-= \\
--\end{array}$ & $\begin{array}{l}1,660 \\
1,693 \\
1,744 \\
1,738 \\
1,688 \\
1,633 \\
1,603 \\
1,618 \\
1,567 \\
1,584 \\
1,571 \\
1,609 \\
1,550 \\
1,559 \\
1,630 \\
1,609 \\
1,551 \\
1,595 \\
1,625 \\
1,546 \\
1,559 \\
1,659 \\
1,697 \\
1,736 \\
1,727 \\
1,762 \\
1,630 \\
1,617\end{array}$ & $\begin{array}{r}119 \\
138 \\
155 \\
191 \\
231 \\
246 \\
291 \\
335 \\
410 \\
458 \\
534 \\
599 \\
612 \\
552 \\
604 \\
645 \\
700 \\
721 \\
908 \\
051 \\
930 \\
931 \\
1,003 \\
1,040 \\
1,071 \\
1,106 \\
1,002 \\
1,108\end{array}$ & $\begin{array}{r}137 \\
147 \\
159 \\
196 \\
219 \\
252 \\
313 \\
366 \\
447 \\
595 \\
618 \\
691 \\
721 \\
655 \\
761 \\
808 \\
660 \\
900 \\
979 \\
1,041 \\
1,107 \\
1,113 \\
1,185 \\
1,231 \\
1,267 \\
1,301 \\
1,295 \\
1,310\end{array}$ & $\begin{array}{r}205 \\
243 \\
275 \\
324 \\
347 \\
372 \\
442 \\
482 \\
566 \\
637 \\
732 \\
025 \\
842 \\
780 \\
869 \\
892 \\
969 \\
1,006 \\
1,077 \\
1,188 \\
1,361 \\
1,299 \\
1,364 \\
1,435 \\
2,465 \\
1,506 \\
1,501 \\
1,488\end{array}$ & $\begin{array}{l}2,383 \\
2,501 \\
3,676 \\
2,785 \\
2,789 \\
2,790 \\
3,865 \\
2,982 \\
3,070 \\
3,285 \\
3,444 \\
3,625 \\
3,626 \\
3,635 \\
3,469 \\
3,938 \\
4,150 \\
4,279 \\
4,461 \\
4,502 \\
4,632 \\
4,827 \\
4,975 \\
5,181 \\
5,293 \\
5,485 \\
5,592 \\
5,571\end{array}$ \\
\hline
\end{tabular}

1) U.8. geographic coverage is the 50 steted and the pietrlet of calmble.

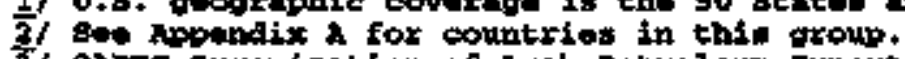

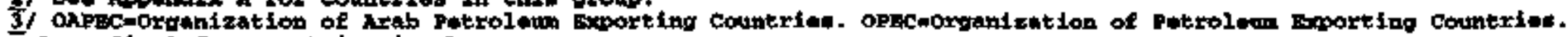

soin nomdix A far countries in these gropips.

Paprelinimaty.

Bouxces: Soe wat of tection it. 
Tablo 4.4 Worla oll supply, 1970-1997

(Thoodand Bircels per Day)

\begin{tabular}{|c|c|c|c|c|c|c|}
\hline & & $\begin{array}{l}\text { Dattend } \\
\text { etatcont/ }\end{array}$ & $\begin{array}{l}\text { Pereing } \\
\text { culfy }\end{array}$ & aspes/ & of $2 x 3$ & world \\
\hline $\begin{array}{l}1970 \\
1971 \\
1972 \\
1973 \\
1974 \\
1975 \\
1976 \\
1977 \\
1978 \\
1979 \\
1990 \\
1981 \\
1989 \\
1989 \\
1984 \\
1985 \\
1996 \\
1947 \\
1980 \\
1989 \\
1990 \\
1991 \\
1992 \\
1993 \\
1994 \\
1995 \\
1996 \\
1997\end{array}$ & 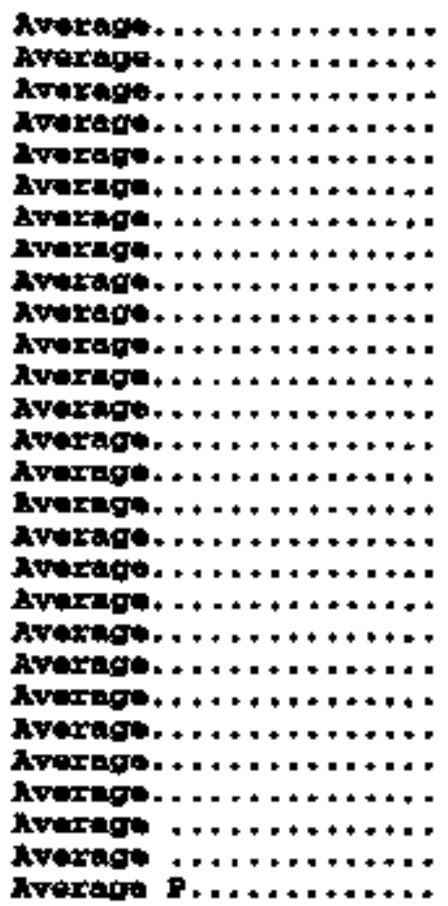 & $\begin{array}{l}11,673 \\
11,554 \\
11,601 \\
11,428 \\
10,978 \\
10,505 \\
10,251 \\
10,437 \\
10,020 \\
10,707 \\
10,809 \\
10,739 \\
10,703 \\
10,780 \\
11,107 \\
11,192 \\
10,905 \\
10,648 \\
10,473 \\
9,800 \\
9,677 \\
9,893 \\
9,769 \\
9,602 \\
9,013 \\
9,400 \\
9,445 \\
9,461\end{array}$ & $\begin{array}{l}13,541 \\
15,940 \\
17,734 \\
20,095 \\
21,590 \\
19,223 \\
21,847 \\
22,098 \\
21,067 \\
21,569 \\
16,541 \\
15,894 \\
12,015 \\
11,676 \\
11,433 \\
10,320 \\
12,141 \\
12,869 \\
14,310 \\
15,733 \\
16,249 \\
15,704 \\
17,010 \\
17,792 \\
18,072 \\
18,351 \\
14,486 \\
19,641\end{array}$ & $\begin{array}{l}14,571 \\
15,627 \\
16,451 \\
18,656 \\
16,399 \\
16,831 \\
19,563 \\
20,372 \\
19,819 \\
22,575 \\
20,715 \\
17,619 \\
13,796 \\
12,416 \\
12,619 \\
11,496 \\
13,653 \\
13,992 \\
15,662 \\
16,656 \\
17,232 \\
16,747 \\
17,871 \\
18,505 \\
19,745 \\
19,069 \\
19,259 \\
20,407\end{array}$ & $\begin{array}{l}23,567 \\
25,514 \\
27,228 \\
31,015 \\
30,762 \\
27,210 \\
30,811 \\
31,443 \\
30,120 \\
31,300 \\
27,419 \\
23,393 \\
19,702 \\
18,352 \\
18,389 \\
17,151 \\
19,310 \\
19,589 \\
21,465 \\
23,323 \\
24,596 \\
24,625 \\
25,818 \\
26,610 \\
27,031 \\
27,566 \\
26,521 \\
29,905\end{array}$ & $\begin{array}{l}46,986 \\
51,766 \\
54,574 \\
59,300 \\
59,391 \\
56,511 \\
61,121 \\
63,565 \\
61,225 \\
66,973 \\
64,152 \\
60,761 \\
58,225 \\
58,054 \\
59,644 \\
59,269 \\
61,769 \\
62,427 \\
64,705 \\
65,692 \\
66,754 \\
66,632 \\
66,960 \\
67,361 \\
68,250 \\
69,960 \\
71,764 \\
74,091\end{array}$ \\
\hline
\end{tabular}

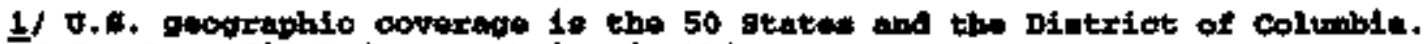

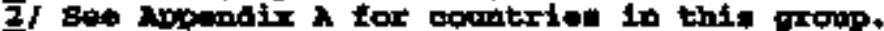

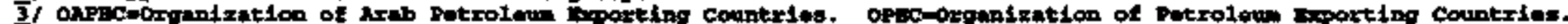

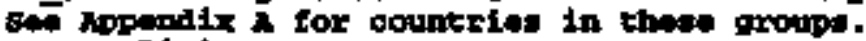

Prexeliminary.

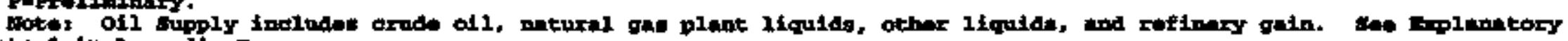
Hote 1 in Nopindix $B$.

Sources: 1 get und of smotion it 


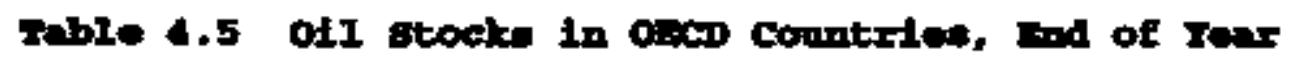
(nillion Darrels)

\begin{tabular}{|c|c|c|c|c|c|c|c|c|c|c|c|}
\hline & & Frxturoe & xtaly & excony & $\begin{array}{l}\text { intted } \\
\text { alngatos }\end{array}$ & 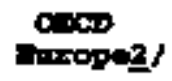 & 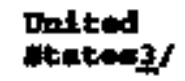 & chrnile & Thpon & otther $1 /$ & $\begin{array}{r}\text { Toted } \\
\text { Orect }\end{array}$ \\
\hline $\begin{array}{l}1973 \\
1974 \\
1975 \\
1976 \\
1977 \\
1978 \\
1979 \\
1980 \\
1991 \\
1902 \\
1983 \\
1984 \\
1985 \\
1986 \\
1987 \\
1988 \\
1909 \\
1990 \\
1991 \\
1992 \\
1993 \\
1994 \\
1995 \\
1996 \\
1997\end{array}$ & 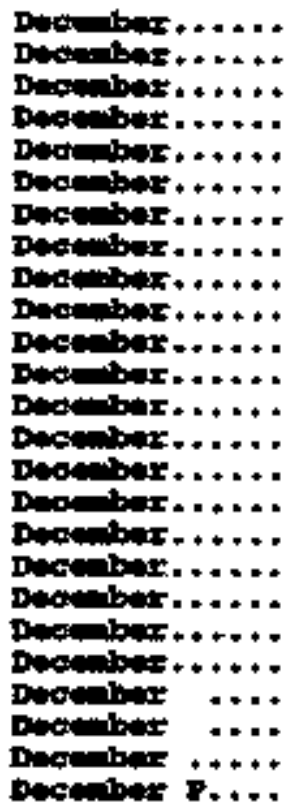 & $\begin{array}{l}201 \\
249 \\
225 \\
234 \\
239 \\
201 \\
216 \\
243 \\
214 \\
193 \\
153 \\
152 \\
139 \\
127 \\
127 \\
140 \\
134 \\
140 \\
153 \\
1 \$ 6 \\
158 \\
150 \\
159 \\
159 \\
164\end{array}$ & $\begin{array}{l}152 \\
167 \\
143 \\
143 \\
161 \\
154 \\
161 \\
170 \\
157 \\
179 \\
149 \\
159 \\
157 \\
155 \\
169 \\
155 \\
164 \\
172 \\
160 \\
174 \\
163 \\
164 \\
162 \\
152 \\
147\end{array}$ & $\begin{array}{l}181 \\
213 \\
187 \\
208 \\
225 \\
290 \\
272 \\
319 \\
297 \\
272 \\
249 \\
239 \\
233 \\
292 \\
259 \\
365 \\
271 \\
265 \\
280 \\
310 \\
309 \\
312 \\
301 \\
300 \\
256\end{array}$ & $\begin{array}{l}158 \\
191 \\
165 \\
165 \\
148 \\
157 \\
169 \\
169 \\
103 \\
129 \\
118 \\
112 \\
123 \\
128 \\
121 \\
112 \\
118 \\
112 \\
119 \\
113 \\
118 \\
118 \\
107 \\
108 \\
105\end{array}$ & 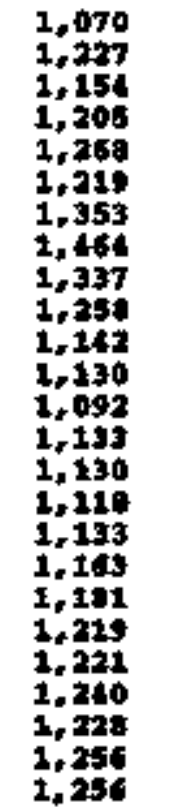 & $\begin{array}{l}1,008 \\
1,074 \\
1,193 \\
1,112 \\
1,312 \\
1,270 \\
1,341 \\
1,392 \\
1,454 \\
1,430 \\
1,454 \\
1,556 \\
1,519 \\
1,593 \\
1,607 \\
1,597 \\
1,591 \\
1,621 \\
1,617 \\
1,597 \\
1,647 \\
1,653 \\
1,563 \\
1,507 \\
1,560\end{array}$ & $\begin{array}{l}140 \\
145 \\
174 \\
193 \\
167 \\
144 \\
150 \\
164 \\
161 \\
136 \\
121 \\
128 \\
125 \\
111 \\
126 \\
116 \\
114 \\
111 \\
119 \\
107 \\
105 \\
119 \\
109 \\
103 \\
115\end{array}$ & $\begin{array}{l}303 \\
370 \\
575 \\
380 \\
409 \\
413 \\
460 \\
495 \\
492 \\
484 \\
470 \\
479 \\
494 \\
509 \\
540 \\
538 \\
577 \\
590 \\
606 \\
603 \\
618 \\
645 \\
630 \\
651 \\
640\end{array}$ & $\begin{array}{l}67 \\
64 \\
67 \\
68 \\
68 \\
68 \\
75 \\
72 \\
67 \\
68 \\
68 \\
69 \\
66 \\
72 \\
71 \\
71 \\
71 \\
73 \\
65 \\
67 \\
69 \\
69 \\
71 \\
74 \\
74\end{array}$ & 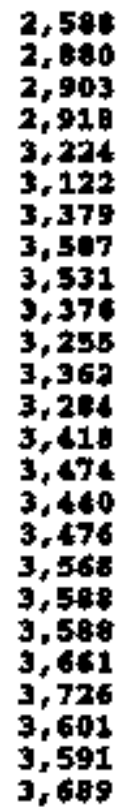 \\
\hline
\end{tabular}

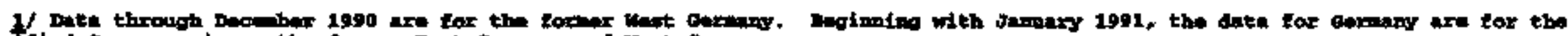

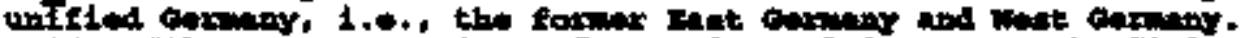

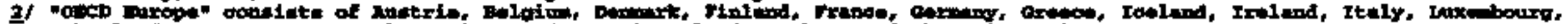

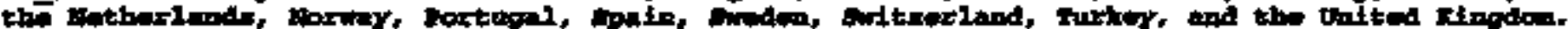

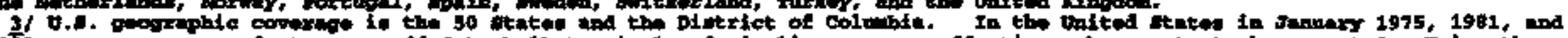

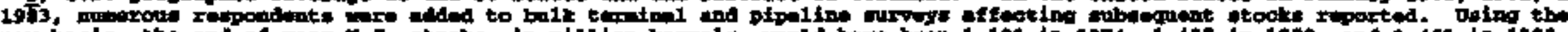

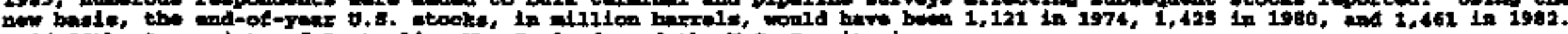

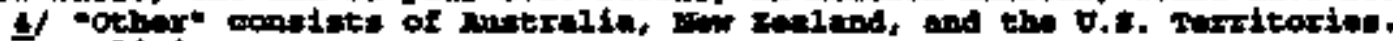

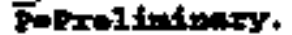

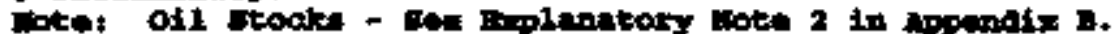

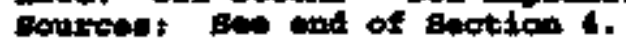


Table 4.6 Woxld oll Dund, 1970-1997

troogind anxrello pax Day

\begin{tabular}{|c|c|c|c|c|c|c|c|c|c|c|c|c|c|c|}
\hline & & France & $\operatorname{Itn} l y$ & Comany 17 & Kingeded & Oxen & $\begin{array}{l}\text { volted } \\
\text { stateas }\end{array}$ & Canadu & Thond & Othore & 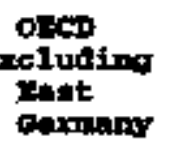 & ant & $\begin{array}{l}\text { Oxep } \\
\text { Inoludind } \\
\text { Eant } \\
\text { Goxpary }\end{array}$ & $\begin{array}{l}\text { Morld } \\
\text { Total }\end{array}$ \\
\hline $\begin{array}{l}1970 \\
1971 \\
1972 \\
1973 \\
1974 \\
1975 \\
1976 \\
1977 \\
1978 \\
1979 \\
1980 \\
1961 \\
1982 \\
1963 \\
1904 \\
1985 \\
19 \$ 6 \\
1967 \\
1988 \\
1989 \\
1990 \\
1991 \\
1992 \\
1993 \\
1994 \\
1995 \\
1996 \\
1997\end{array}$ & 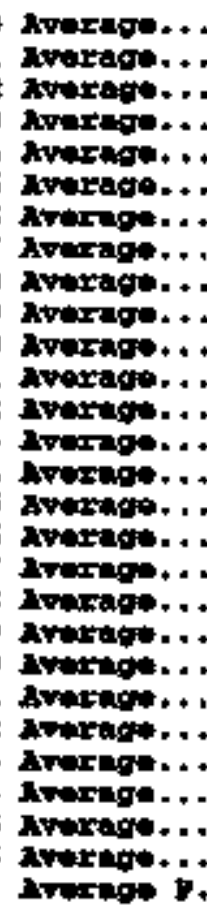 & $\begin{array}{r}1,937 \\
: 2,115 \\
: 2,322 \\
2,601 \\
2,447 \\
: 2,252 \\
: 2,420 \\
2,294 \\
: 2,404 \\
: 2,463 \\
: 2,256 \\
: 1,023 \\
: 1,800 \\
: 1,035 \\
: 1,754 \\
: 1,775 \\
: 1,772 \\
: 1,769 \\
: 1,797 \\
: 1,097 \\
: 1,816 \\
: 1,995 \\
: 1,926 \\
: 1,875 \\
: 1,833 \\
: 1,896 \\
: 1,935 \\
1,935\end{array}$ & $\begin{array}{l}1,710 \\
1,831 \\
1,947 \\
2,068 \\
2,004 \\
1,855 \\
1,971 \\
1,897 \\
1,952 \\
2,039 \\
1,934 \\
1,874 \\
1,781 \\
1,750 \\
1,646 \\
1,717 \\
1,738 \\
1,855 \\
1,836 \\
1,930 \\
1,872 \\
1,863 \\
1,937 \\
1,852 \\
1,841 \\
2,046 \\
2,036 \\
2,045\end{array}$ & $\begin{array}{r}2,607 \\
2,702 \\
2,859 \\
3,055 \\
2,748 \\
2,650 \\
2,877 \\
2,865 \\
2,927 \\
3,003 \\
2,707 \\
2,449 \\
2,372 \\
2,324 \\
2,322 \\
2,330 \\
2,496 \\
2,424 \\
2,422 \\
2,280 \\
2,392 \\
2,020 \\
2,843 \\
2,900 \\
2,679 \\
2,878 \\
2,911 \\
112,904\end{array}$ & $\begin{array}{l}2,096 \\
2,141 \\
2,280 \\
2,341 \\
2,210 \\
1,911 \\
1,992 \\
1,905 \\
1,930 \\
1,971 \\
1,725 \\
1,590 \\
1,590 \\
1,531 \\
1,949 \\
1,634 \\
1,649 \\
1,603 \\
1,697 \\
1,739 \\
1,752 \\
1,601 \\
1,803 \\
1,615 \\
1,897 \\
1,645 \\
1,648 \\
1,199\end{array}$ & $\begin{array}{r}12,404 \\
13,005 \\
13 ; 934 \\
14 ; 925 \\
13,998 \\
13,217 \\
14,124 \\
13,916 \\
10,290 \\
14 ; 667 \\
13,534 \\
12,515 \\
12,053 \\
11,765 \\
11,736 \\
11,641 \\
12,102 \\
12,255 \\
12,427 \\
12,531 \\
12,629 \\
13,391 \\
19,605 \\
13,523 \\
13,597 \\
14,190 \\
14,269 \\
114,439\end{array}$ & $\begin{array}{l}14,697 \\
15,213 \\
16,367 \\
17,309 \\
16,653 \\
16,322 \\
17,461 \\
18,431 \\
18,867 \\
18,513 \\
17,056 \\
16,058 \\
15,296 \\
15,231 \\
15,726 \\
15,726 \\
16,281 \\
16,655 \\
17,283 \\
17,325 \\
16,986 \\
16,714 \\
17,033 \\
17,237 \\
17,719 \\
17,725 \\
18,309 \\
18,630\end{array}$ & $\begin{array}{l}1,516 \\
1,565 \\
1,664 \\
1,729 \\
1,779 \\
1,779 \\
1,819 \\
1,850 \\
1,902 \\
1,971 \\
1,673 \\
1,769 \\
1,579 \\
1,440 \\
1,472 \\
1,504 \\
1,506 \\
1,540 \\
1,693 \\
1,733 \\
1,690 \\
1,622 \\
1,643 \\
1,680 \\
1,727 \\
1,755 \\
1,797 \\
1,856\end{array}$ & 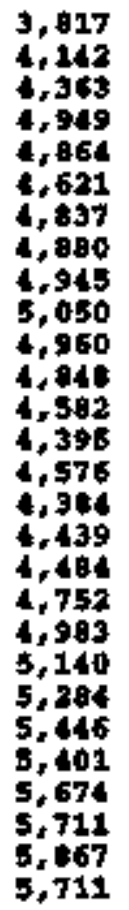 & $\begin{array}{r}836 \\
691 \\
949 \\
988 \\
1,095 \\
3,011 \\
1,119 \\
1,160 \\
1,204 \\
1,176 \\
1,072 \\
1,000 \\
1,000 \\
953 \\
990 \\
976 \\
951 \\
959 \\
939 \\
996 \\
1,027 \\
3,056 \\
1,041 \\
1,110 \\
1,174 \\
1,249 \\
1,191 \\
1,130\end{array}$ & $\begin{array}{r}33,270 \\
31,759 \\
37,275 \\
39,900 \\
38,379 \\
36,980 \\
39,358 \\
40,737 \\
41,187 \\
41,379 \\
38,595 \\
36,269 \\
34,517 \\
33,793 \\
34,500 \\
34,271 \\
35,279 \\
33,911 \\
37,093 \\
37,370 \\
37,475 \\
=- \\
=- \\
=- \\
=- \\
=- \\
=\end{array}$ & $\begin{array}{l}223 \\
239 \\
270 \\
282 \\
307 \\
307 \\
329 \\
347 \\
363 \\
369 \\
375 \\
345 \\
371 \\
336 \\
340 \\
362 \\
362 \\
342 \\
322 \\
300 \\
292 \\
-- \\
=- \\
=- \\
=- \\
=- \\
-\pi\end{array}$ & 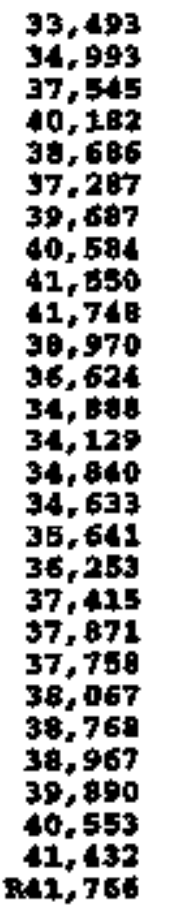 & $\begin{array}{r}16,609 \\
49,, 116 \\
53,094 \\
57,237 \\
56,677 \\
56,198 \\
59,673 \\
61,826 \\
64,158 \\
65,220 \\
63,067 \\
60,903 \\
59,503 \\
58,739 \\
59,831 \\
60,091 \\
61,759 \\
62,999 \\
64,919 \\
65,917 \\
65,995 \\
66,577 \\
66,742 \\
67,043 \\
69,913 \\
69,926 \\
71,524 \\
173,313\end{array}$ \\
\hline
\end{tabular}

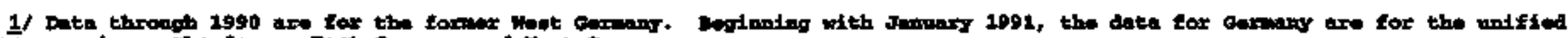

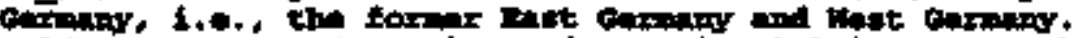

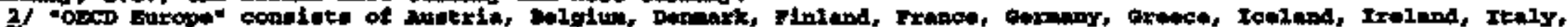

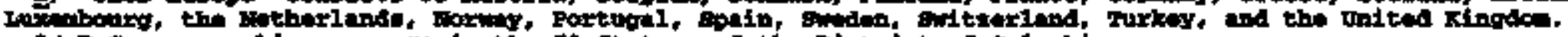

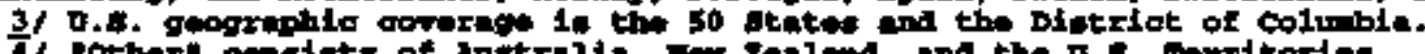

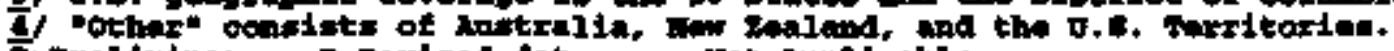

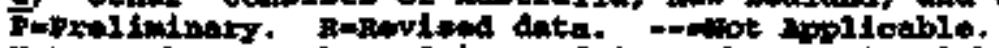

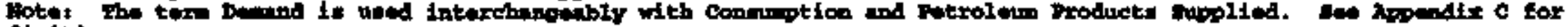
dinglition.

Bcourcall : Doo ond of section 4. 
rable 4.7 0.200 Total not I-morte, 1987-1997

(millica Burrele per Dar)

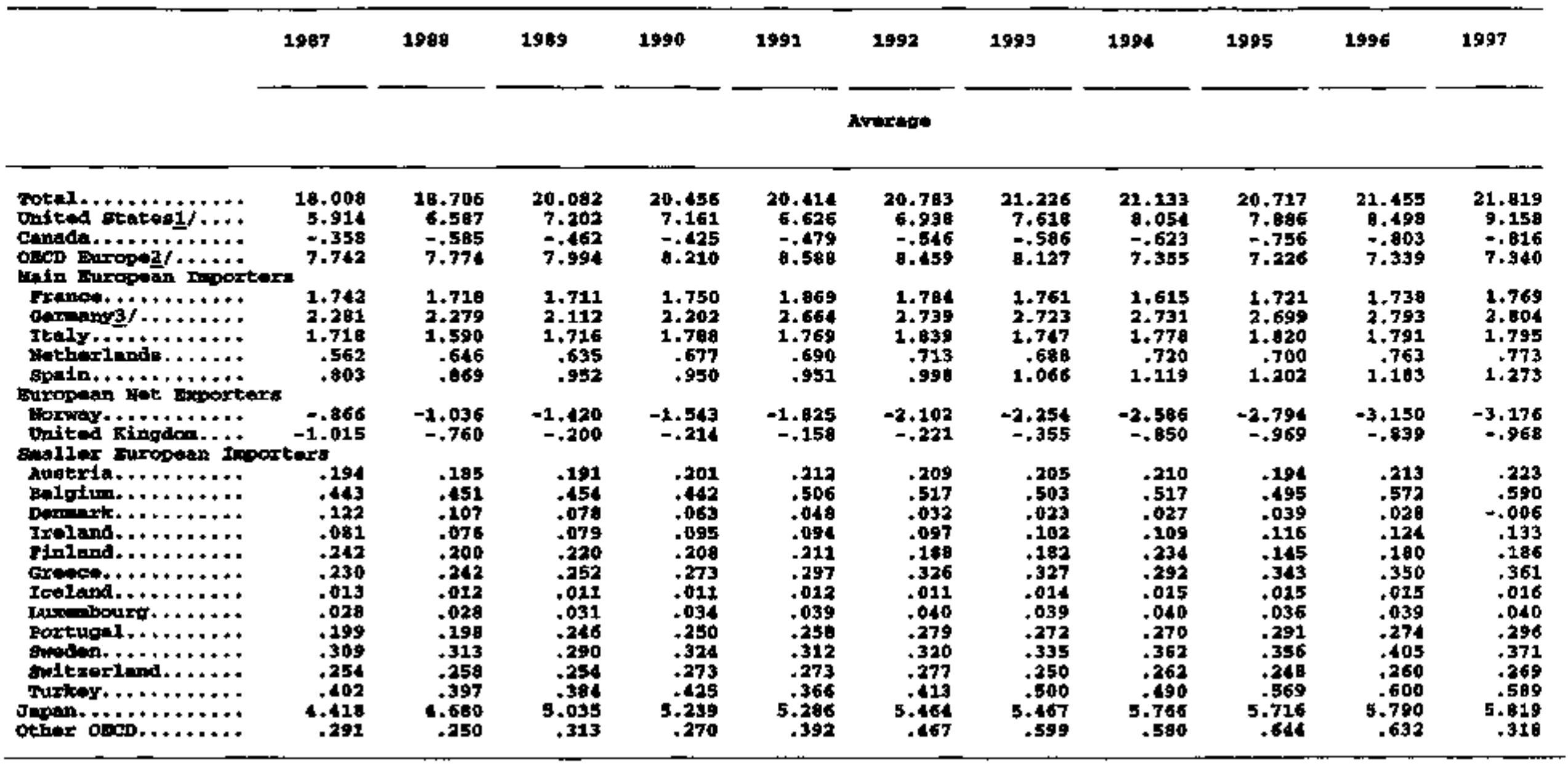

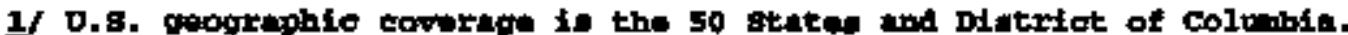

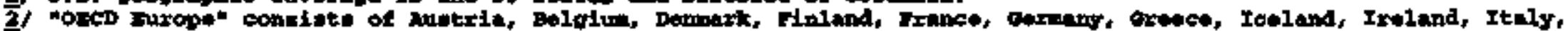

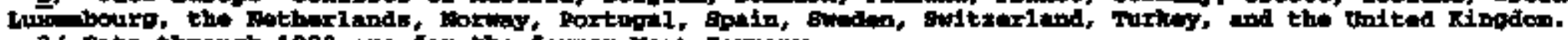

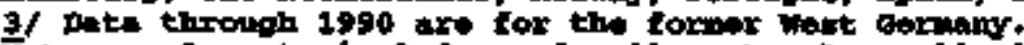

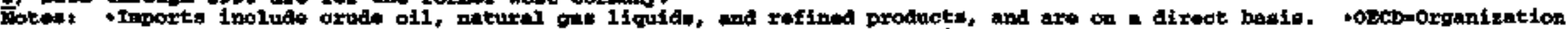

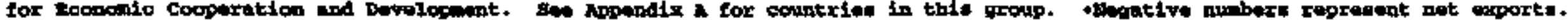

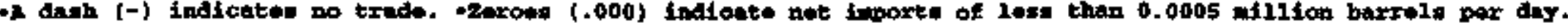

Date tor 1995 ere pralsinnry.

Bourtes: ter end of Aetion 4. 
Table 4.6 orco Irporte from optic, 1987-1997

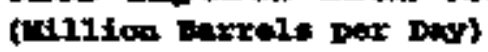

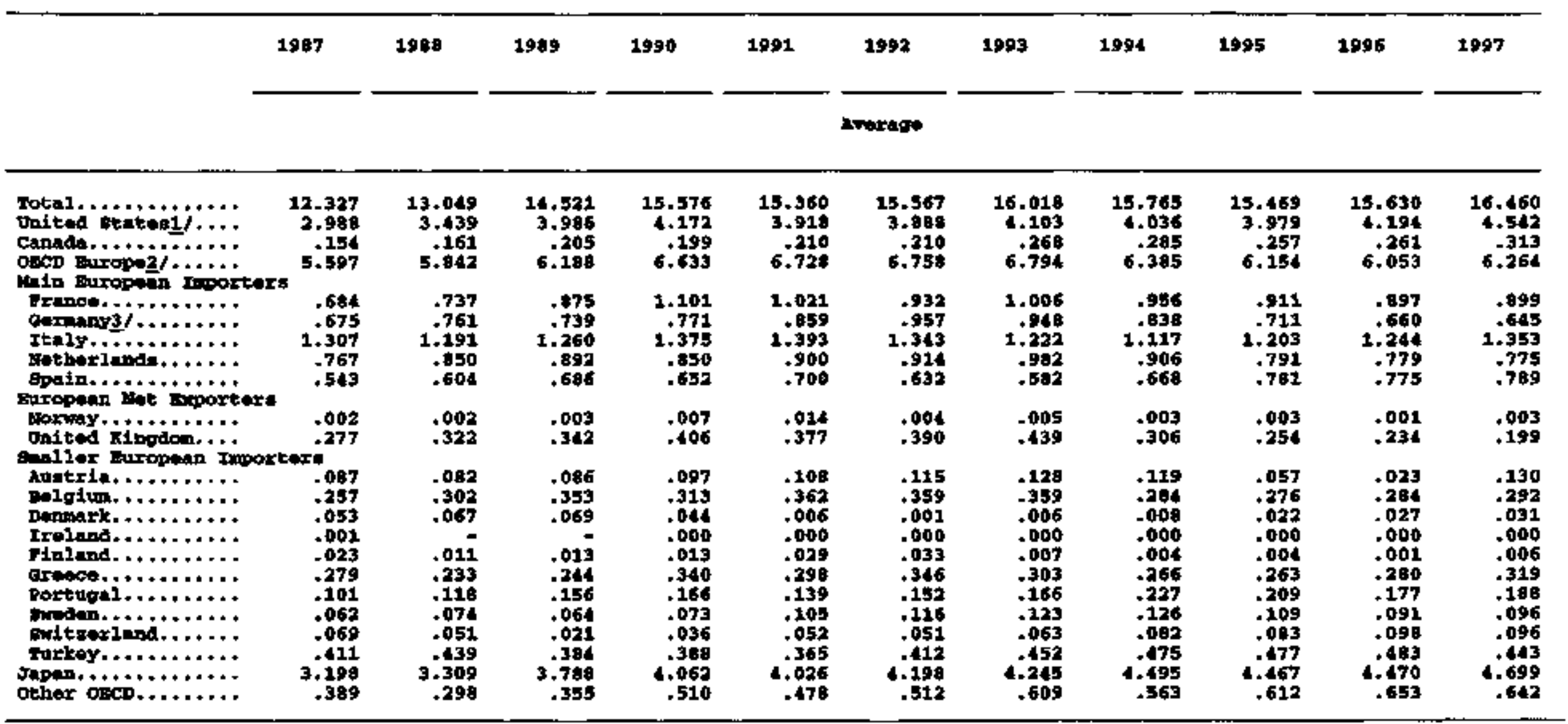

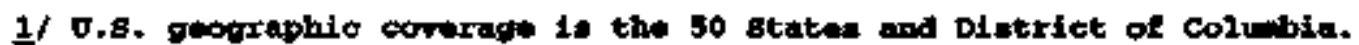

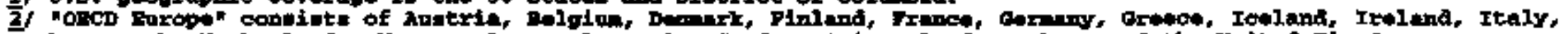

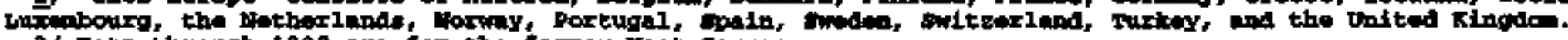

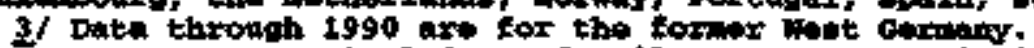

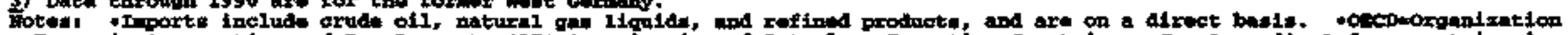

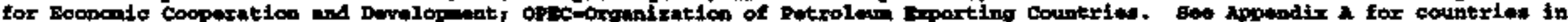

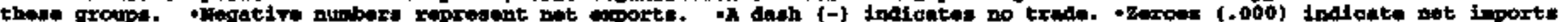

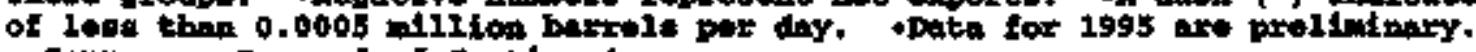

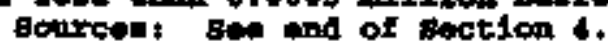




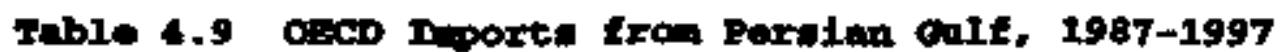

(H111ice Burrole por Day)

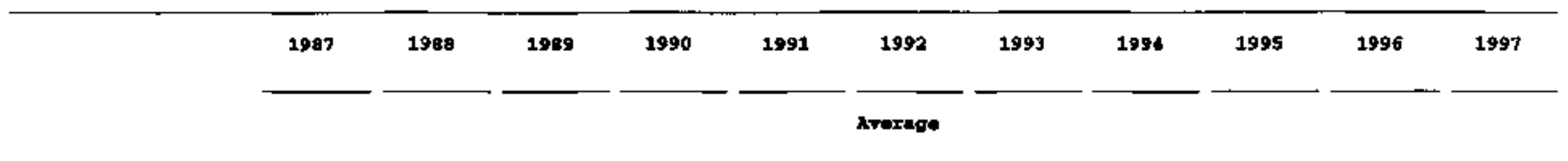

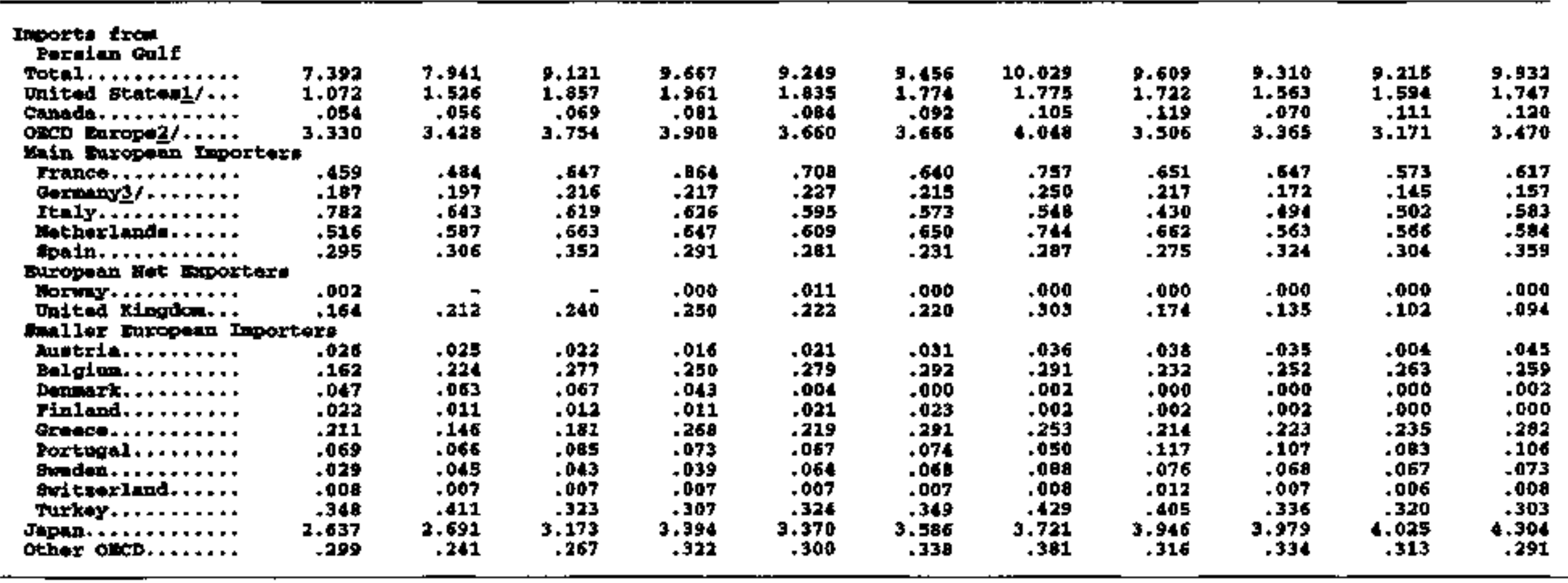

1/ 0. . . geographic copernge is the so ateter and Dietrict of colunbia.

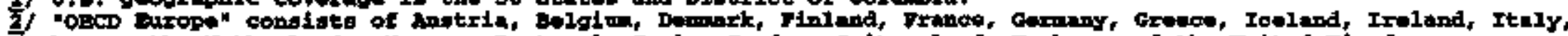

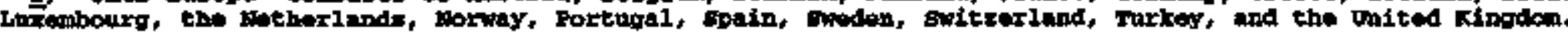

$3 /$ Data through 1990 are for the former Hapt Gerrany.

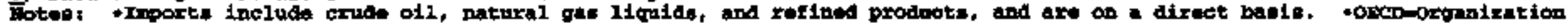

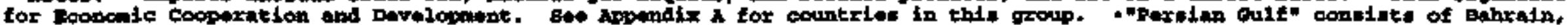

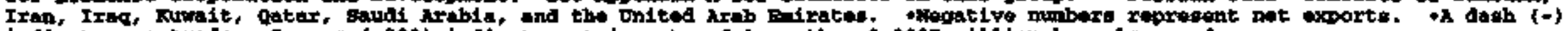

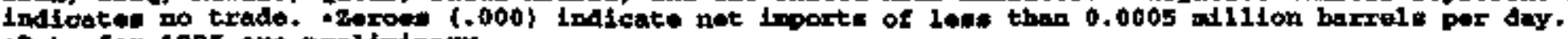

- thete for 1995 are proliviniry.

pourcer soe ond of pection 4. 


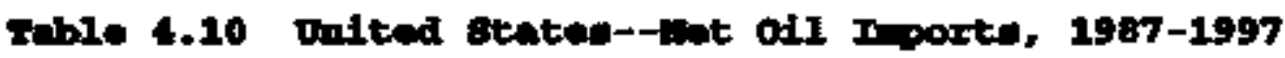

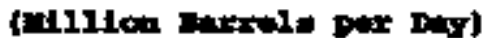

\begin{tabular}{|c|c|c|c|c|c|c|c|c|c|c|c|}
\hline & 1987 & 1900 & $19 t 9$ & $19 \$ 0$ & $10 * 1$ & $19+2$ & 1993 & 1994 & 1995 & 1996 & 1957 \\
\hline & \multicolumn{11}{|c|}{ Avarage } \\
\hline Jotal Not I I & $\begin{array}{l}5.914 \\
2.908\end{array}$ & $\begin{array}{l}6.587 \\
3.439\end{array}$ & $\begin{array}{l}7.202 \\
3.986\end{array}$ & $\begin{array}{l}7.161 \\
4.172\end{array}$ & $\begin{array}{l}6.626 \\
3.918\end{array}$ & $\begin{array}{l}6.938 \\
3.888\end{array}$ & $\begin{array}{l}7.610 \\
4.103\end{array}$ & $\begin{array}{l}8.054 \\
4.036\end{array}$ & $\begin{array}{l}7.806 \\
3.979\end{array}$ & $\begin{array}{l}8.498 \\
4.194\end{array}$ & $\begin{array}{l}9.158 \\
4.542\end{array}$ \\
\hline 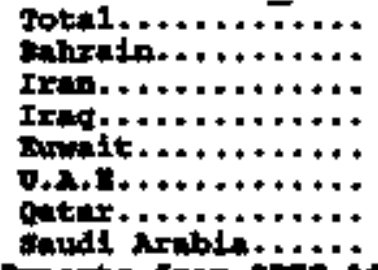 & $\begin{array}{r}1.073 \\
-.001 \\
.095 \\
.083 \\
.019 \\
.058 \\
.000 \\
.751\end{array}$ & $\begin{array}{r}1.526 \\
.000 \\
.000 \\
.343 \\
.092 \\
.028 \\
.000 \\
1.069\end{array}$ & $\begin{array}{r}1.057 \\
-.001 \\
.000 \\
.449 \\
.157 \\
.027 \\
.002 \\
1.224\end{array}$ & $\begin{array}{r}1.961 \\
-.001 \\
.000 \\
.518 \\
.086 \\
.016 \\
.004 \\
1.339\end{array}$ & $\begin{array}{r}1.855 \\
-.001 \\
.032 \\
.000 \\
.006 \\
.001 \\
.000 \\
1.797\end{array}$ & $\begin{array}{r}1.774 \\
. .002 \\
.000 \\
.000 \\
.051 \\
.004 \\
.001 \\
1.720\end{array}$ & $\begin{array}{r}1.775 \\
=.002 \\
.000 \\
.000 \\
.353 \\
.009 \\
.001 \\
1.414\end{array}$ & $\begin{array}{r}1.722 \\
=.002 \\
.000 \\
.000 \\
.312 \\
.012 \\
.000 \\
1.002\end{array}$ & $\begin{array}{r}1.563 \\
-.003 \\
.000 \\
.000 \\
.218 \\
.004 \\
.000 \\
1.344\end{array}$ & $\begin{array}{r}1.594 \\
-.002 \\
.000 \\
.001 \\
.236 \\
-.009 \\
.000 \\
1.363\end{array}$ & $\begin{array}{r}1.767 \\
-.002 \\
.000 \\
.089 \\
.253 \\
-.003 \\
.004 \\
1.006\end{array}$ \\
\hline 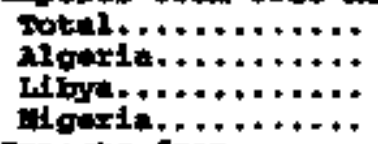 & $\begin{array}{l}.030 \\
.295 \\
.090 \\
.535\end{array}$ & $\begin{array}{l}.910 \\
.300 \\
.000 \\
.618\end{array}$ & $\begin{array}{r}1.004 \\
.269 \\
.000 \\
.915\end{array}$ & $\begin{array}{r}1.000 \\
.280 \\
.000 \\
.000\end{array}$ & $\begin{array}{l}.956 \\
.253 \\
.000 \\
.703\end{array}$ & $\begin{array}{l}.976 \\
.196 \\
.000 \\
.680\end{array}$ & $\begin{array}{l}.956 \\
.220 \\
.000 \\
.736\end{array}$ & $\begin{array}{l}.080 \\
.243 \\
.000 \\
.637\end{array}$ & $\begin{array}{l}.859 \\
.234 \\
.000 \\
.625\end{array}$ & $\begin{array}{l}. \$ 72 \\
.256 \\
.000 \\
.616\end{array}$ & $\begin{array}{r}.979 \\
.285 \\
.000 \\
.694\end{array}$ \\
\hline 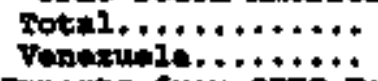 & $\begin{array}{l}+901 \\
.901\end{array}$ & .790 & $\begin{array}{l}.861 \\
.061\end{array}$ & $\begin{array}{l}1.016 \\
1.016\end{array}$ & $\begin{array}{l}1.020 \\
1.020\end{array}$ & $\begin{array}{l}1.161 \\
1.161\end{array}$ & $\begin{array}{l}1.295 \\
1.295\end{array}$ & $\begin{array}{l}1.322 \\
1.322\end{array}$ & $\begin{array}{l}1.468 \\
1.468\end{array}$ & $\begin{array}{l}1.667 \\
1.667\end{array}$ & $\begin{array}{l}1.758 \\
2.758\end{array}$ \\
\hline Iothen $\ldots \ldots \ldots \ldots$ & $\begin{array}{l}.284 \\
.284\end{array}$ & .205 & $\begin{array}{l}.182 \\
.182\end{array}$ & .113 & $\begin{array}{l}.106 \\
.106\end{array}$ & .075 & .074 & $\begin{array}{l}.109 \\
+109\end{array}$ & $\begin{array}{r}.087 \\
.007\end{array}$ & .058 & $\begin{array}{r}.057 \\
.057\end{array}$ \\
\hline
\end{tabular}

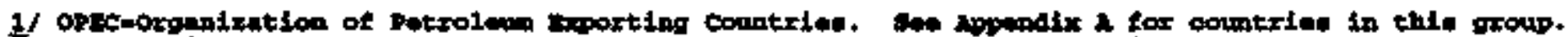

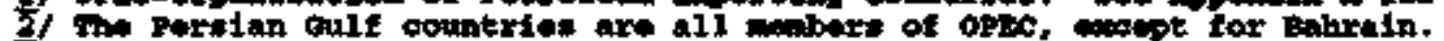

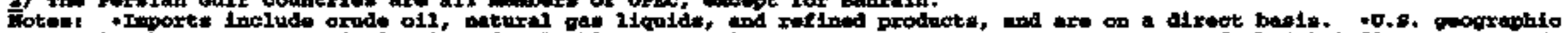

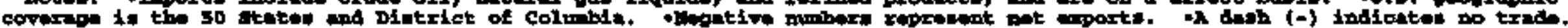

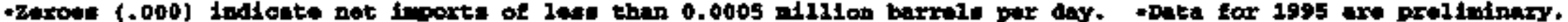

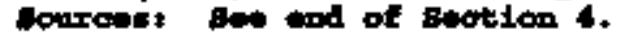


rable 4+11 Japan--Fot oil Imorts, 1987-1997

(Willton Baxrule par Dor)

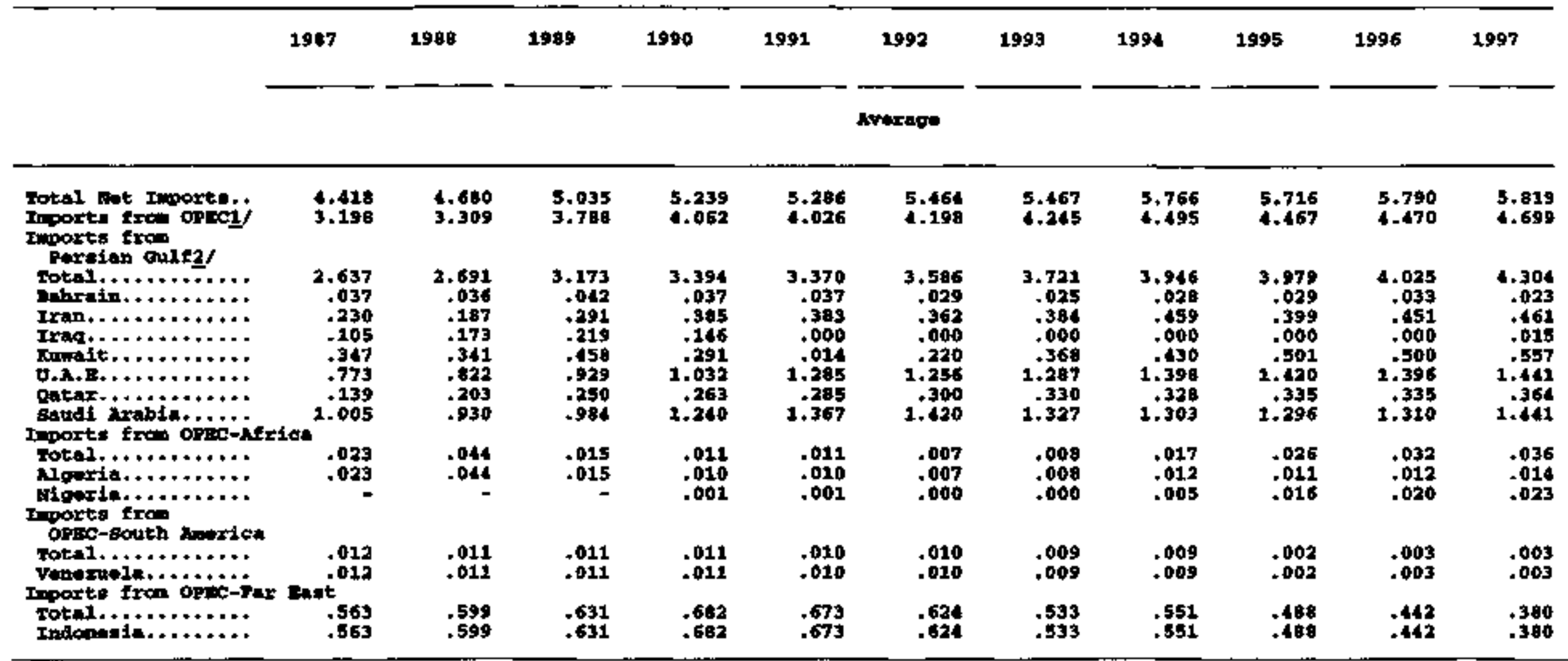

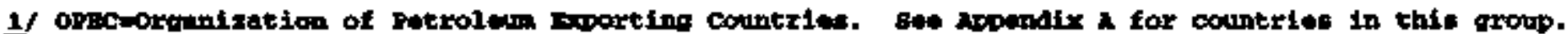

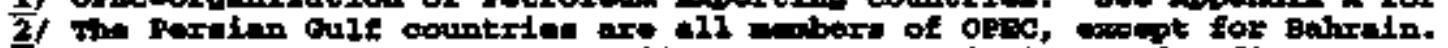

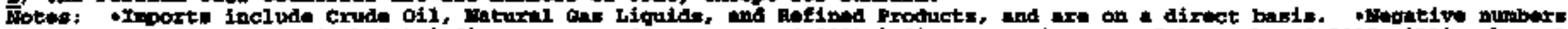

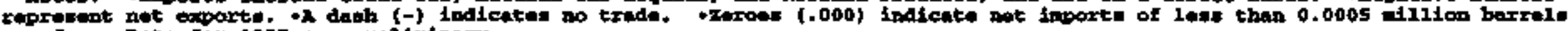
bar dyy. Daten for 1995 are prolimjnary.

gourosed bere end of deotion 4 . 
Toble 4.12 OACD Europe-1.10t O11 Iporte, 1987-1997

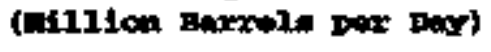

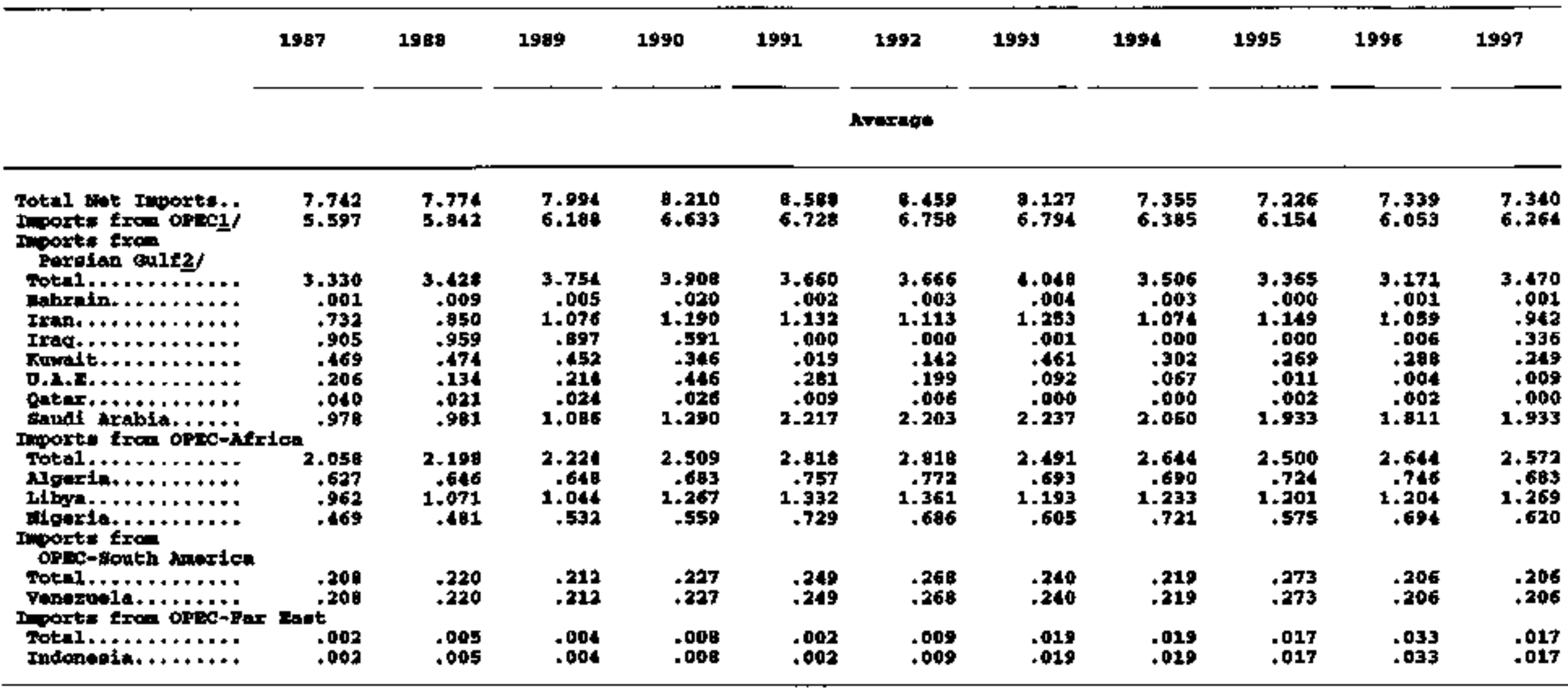

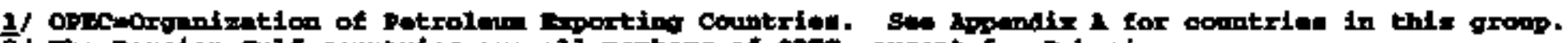

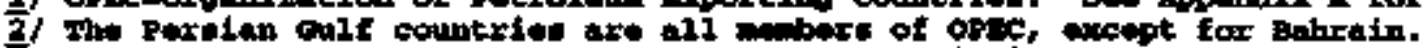

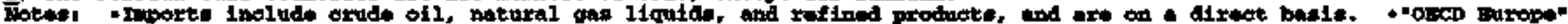

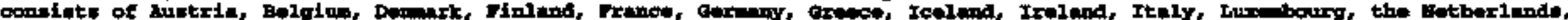

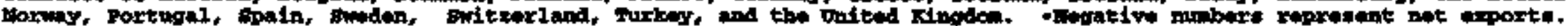

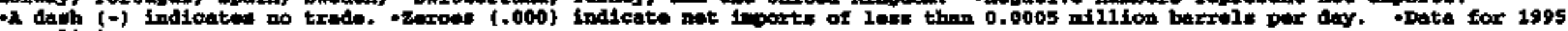
are prolininiry.

ponzses: ser and of section if 
Table 4.13 Franoe-10t oll Theorts, 1987-1997

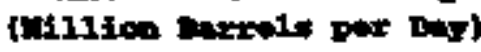

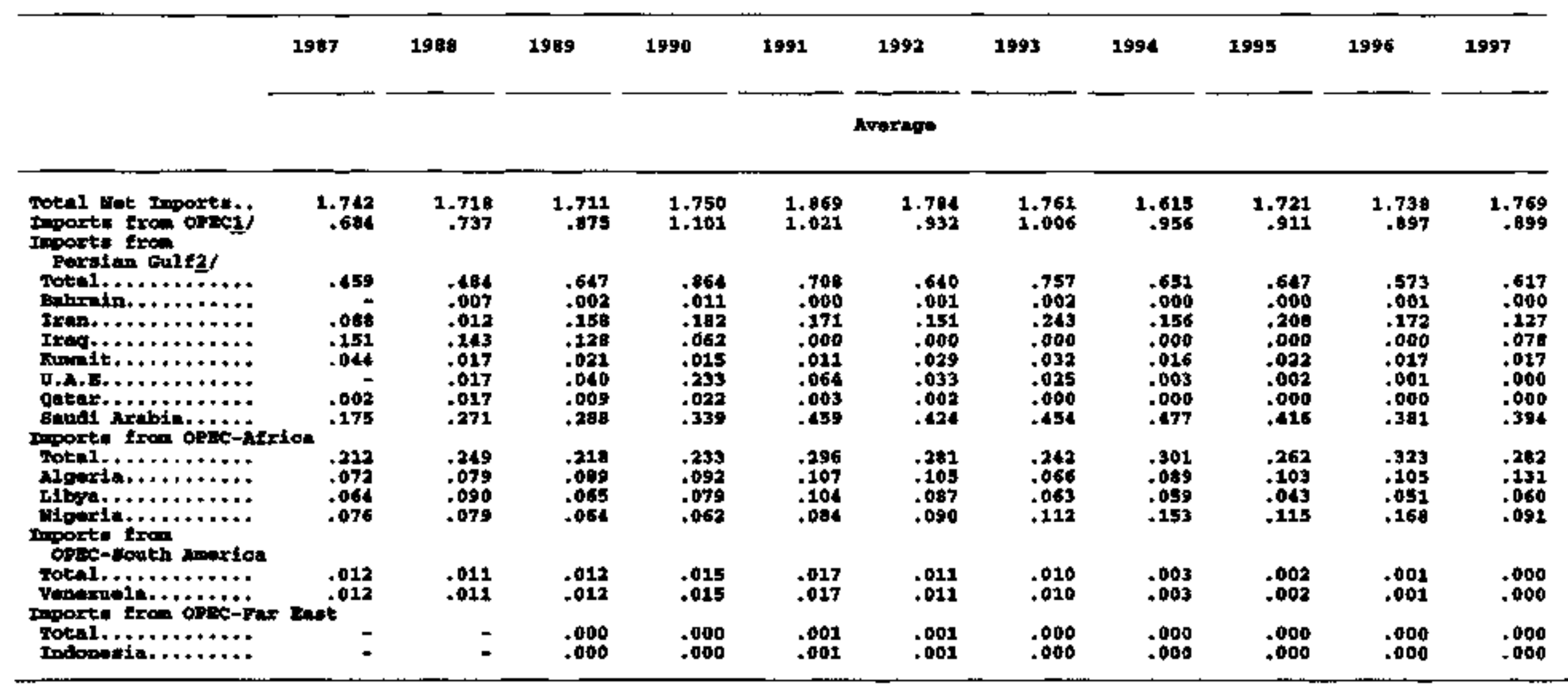

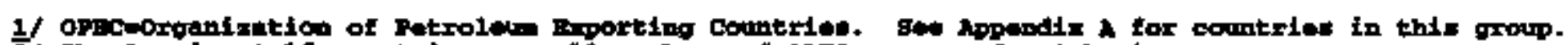

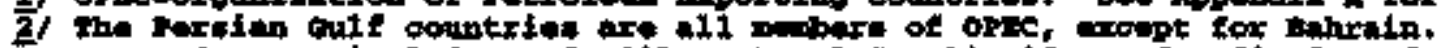

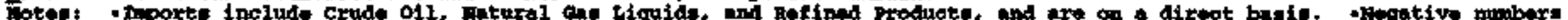

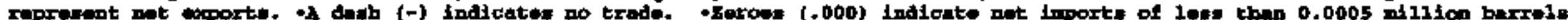
par day. Dnte for 1995 exe prelindmary.

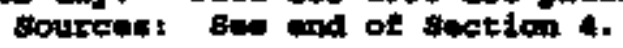


Toble 4.14 Gamany--17ot oil T-ports, 1997-1997 (allien barwale per Der)

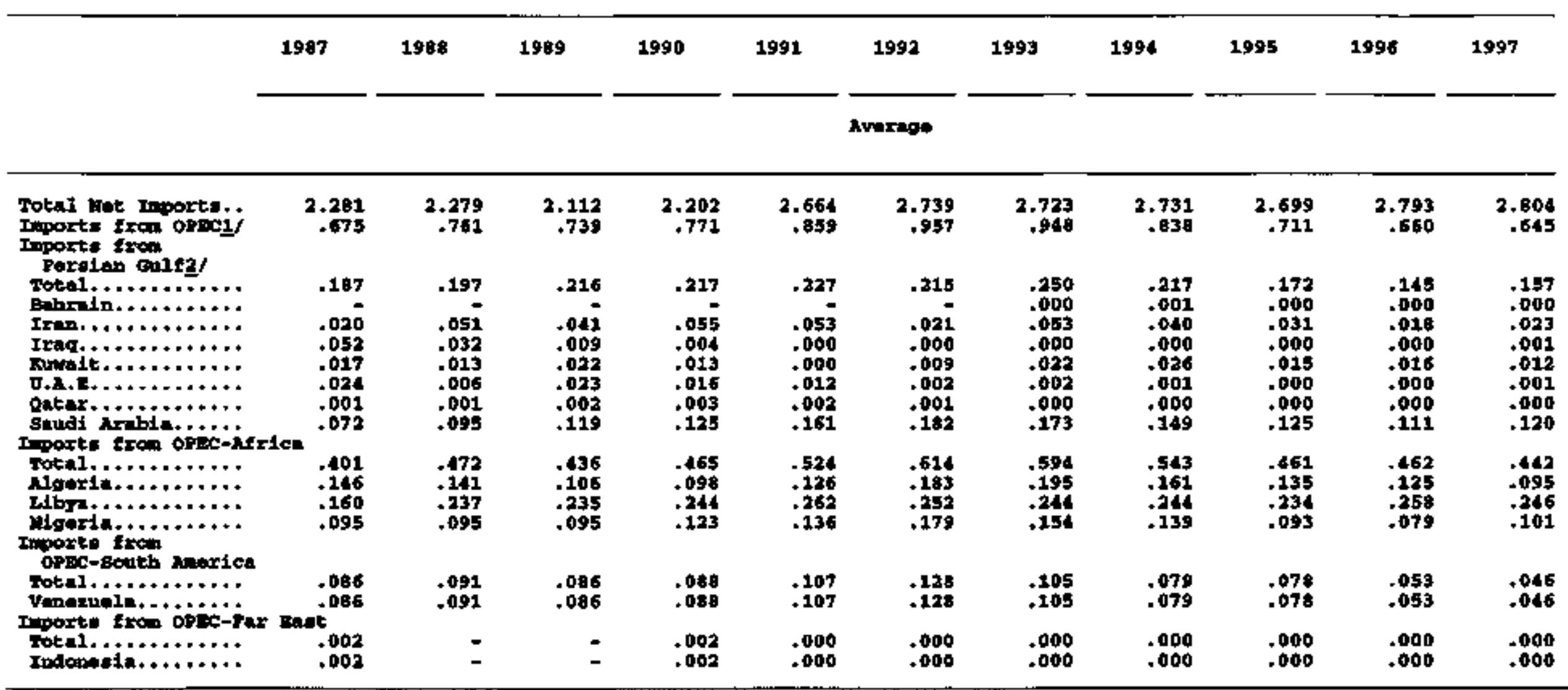

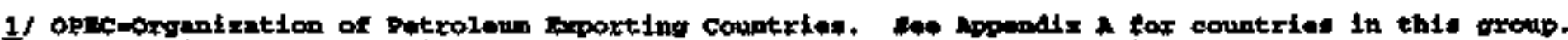

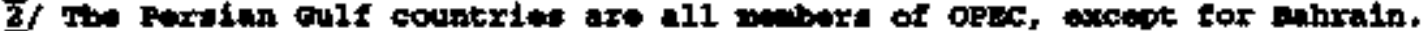

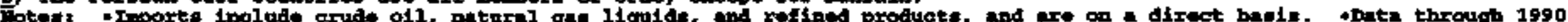

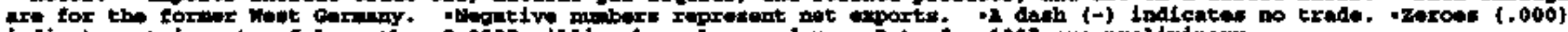

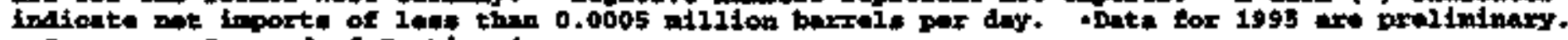

sourois: ser and of section 4 . 
Tablo 4.15 rtaly--10t OS1 Hports, 1987-1997 (ililfon Binrets par Dos)

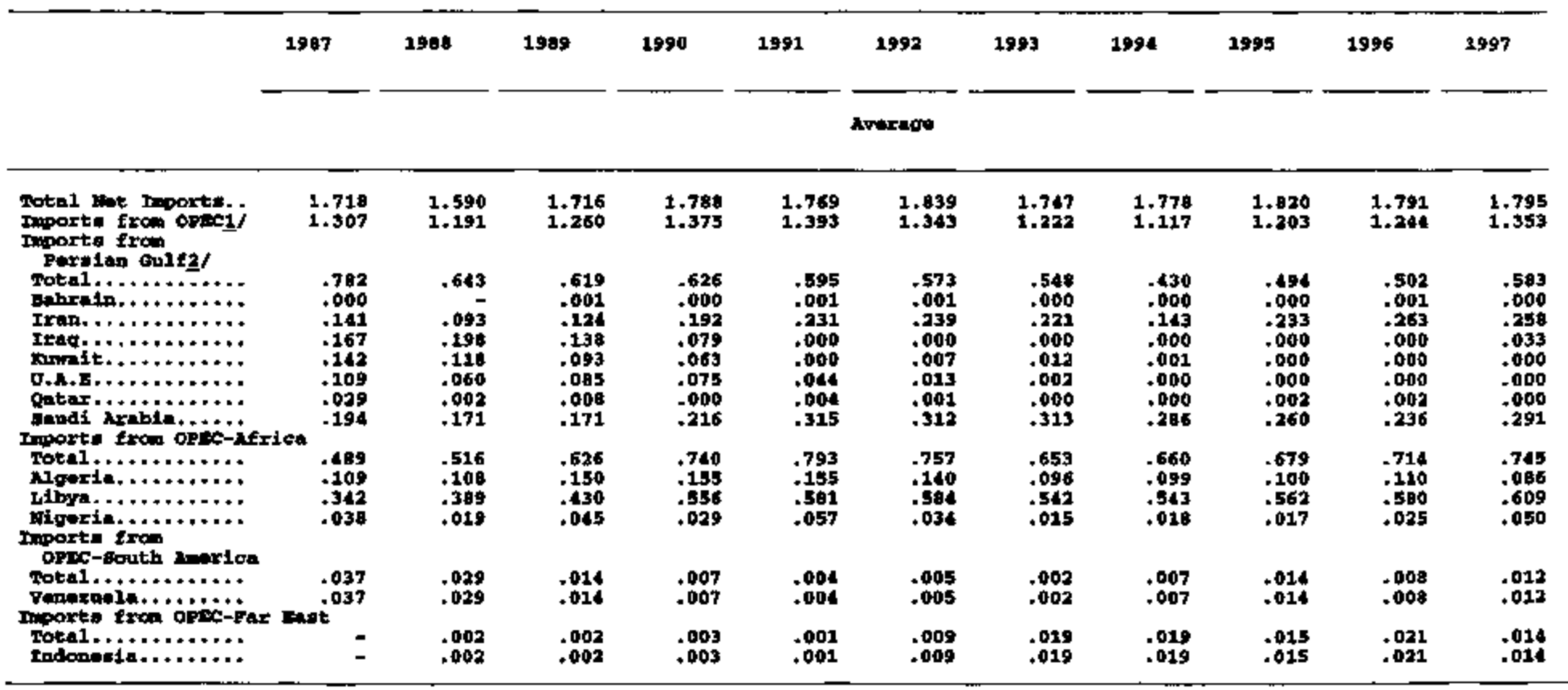

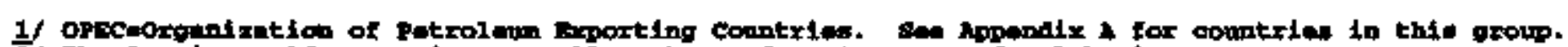

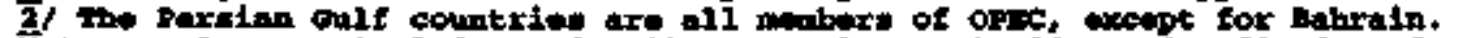

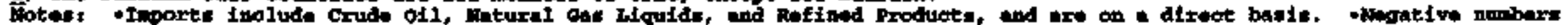

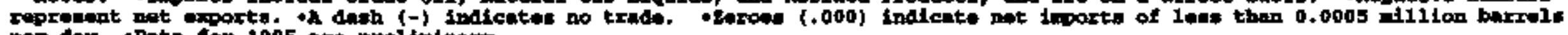

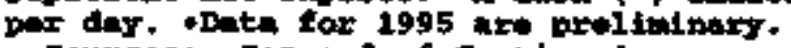

Borrobst ser ind of gention 4 . 
Table 4.16 C.CD Imorts fron Ireg, 1967-1997

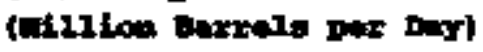

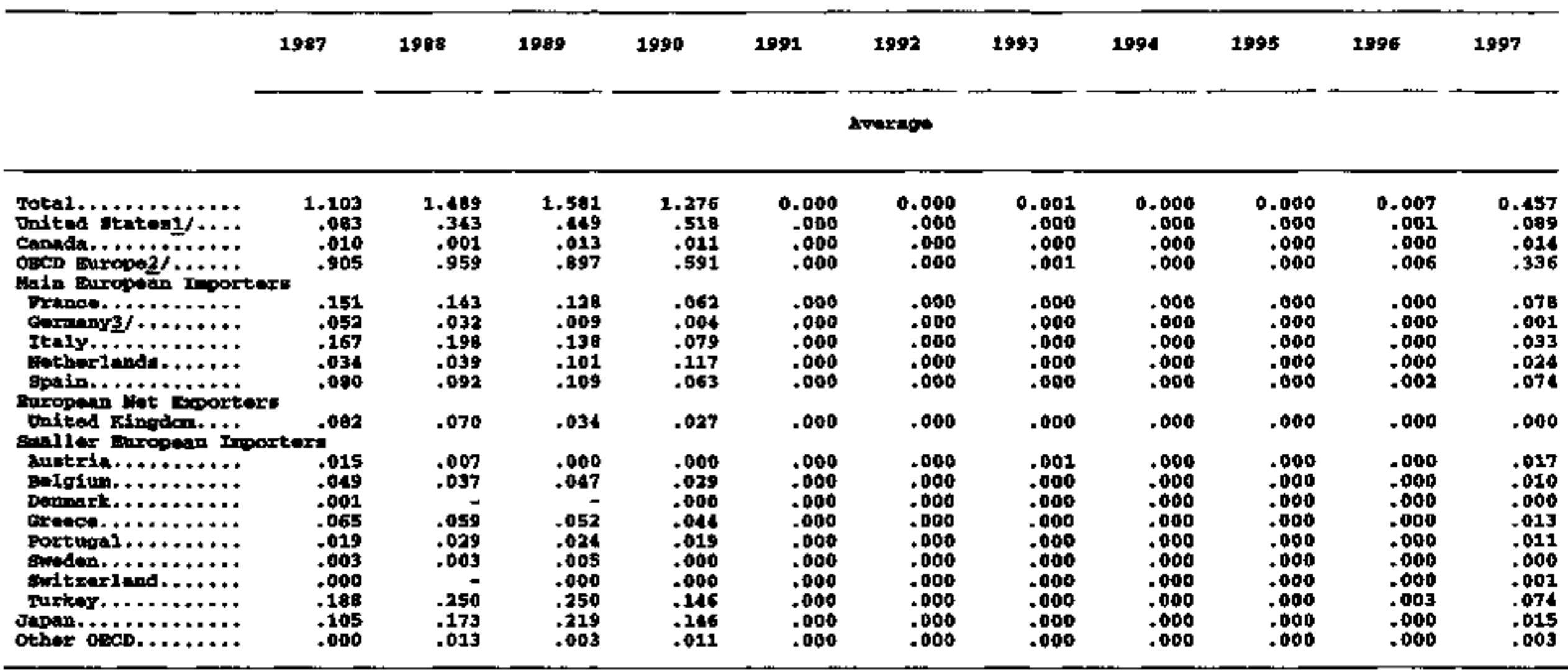

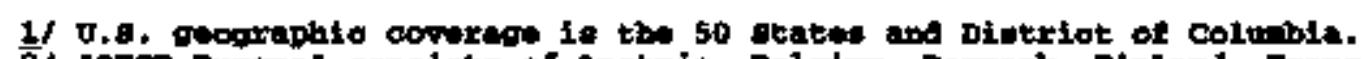

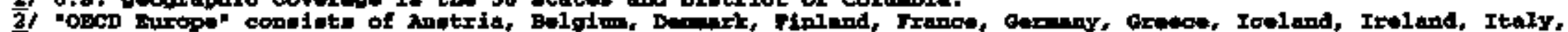

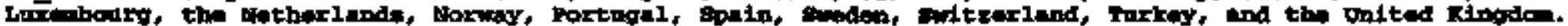

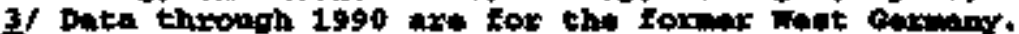

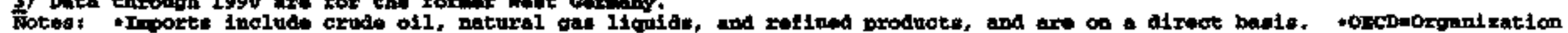

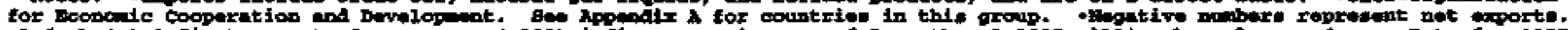

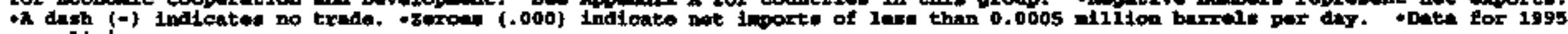
axt preliminary.

Bourots, end of ention 4 . 


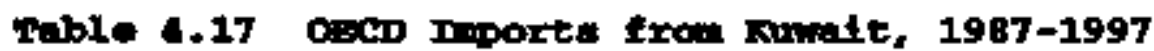
(Milian Rarrolle post pay)

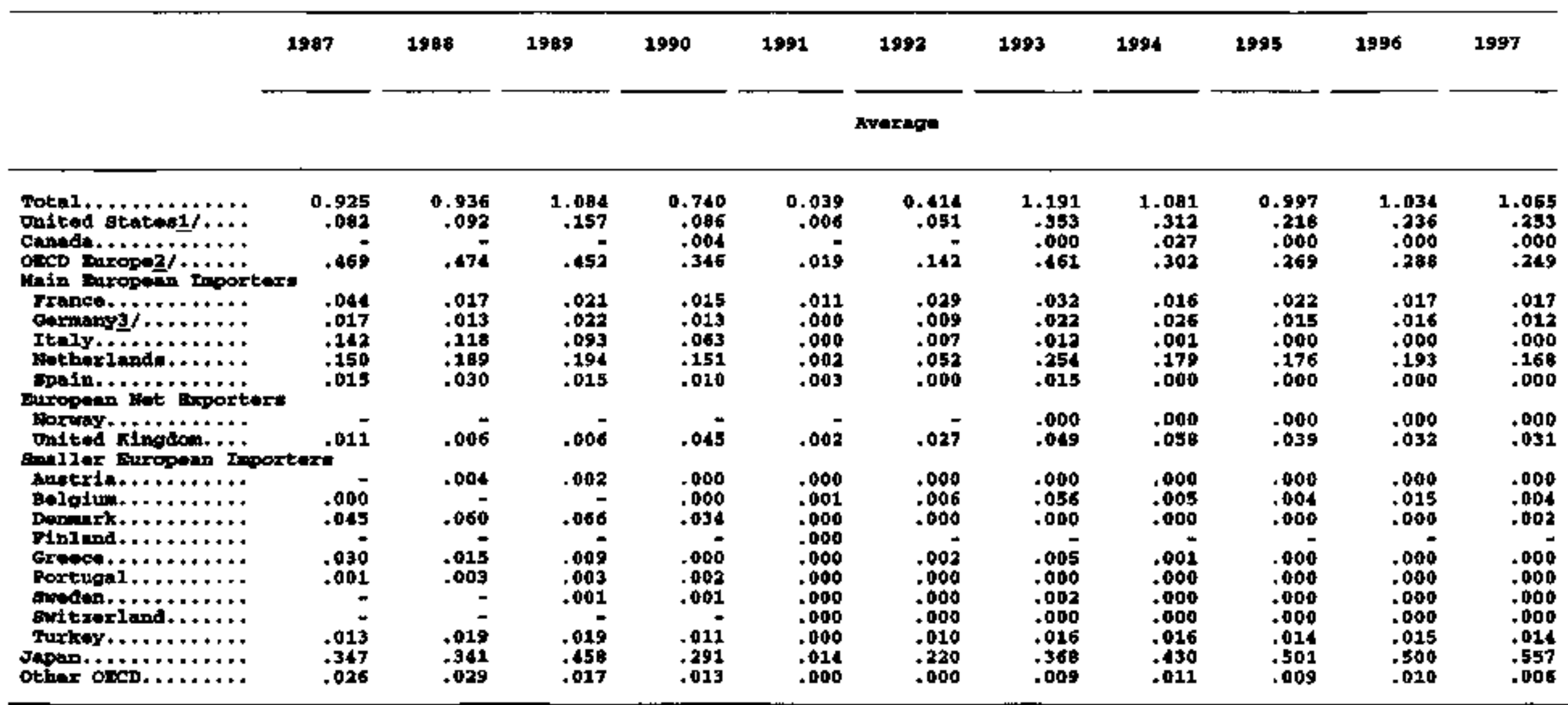

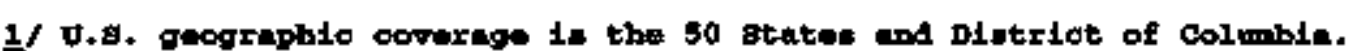

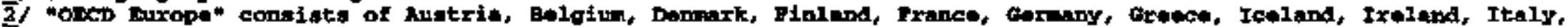

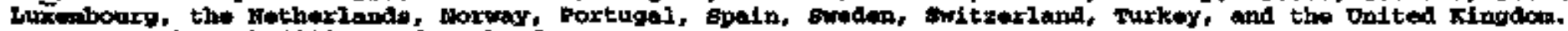

$3 /$ Dnte through 1990 are for the formir wett eerming.

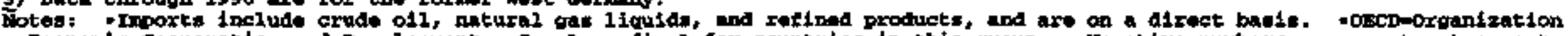

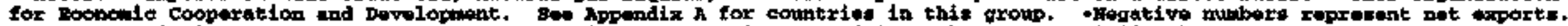

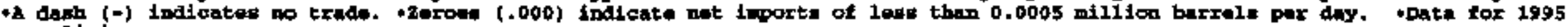
are prolindnery.

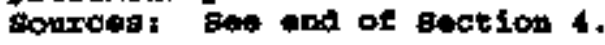




\section{Sources for Section 4 (Historical Oil Data)}

Crode Oil Production, Natmal Gas Liquudds Production, Other Liquidts, and Refinery Gain

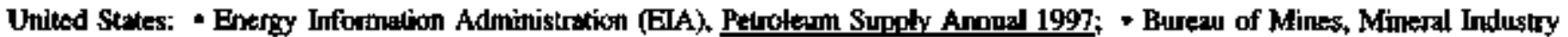
Surveys, Petroleum Statement, Annusl, varioes issues.

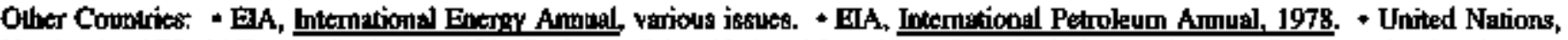
Yearbook of Wordd Energy Statistics, 1979. Refinery Gains: Nexional Petroleum Council, U.S. Perrobum Refinery Study, October J986.

\section{Oil Stocks}

United States: ElA, Petrodeum Supply Annud 1997.

Other OECD Comtries: - 1973-1982: Organizalion for Economic Cooperation and Development (OECD), Ouarterly Oil Statistics and Energy Falances various issues. 1983-1997: OECD, Monthly Ol Satistics in the EIA, Intemational Energy Database.

\section{Oil Demand}

United Sistes: ElA, Petroleum Supply Anmual 1997.

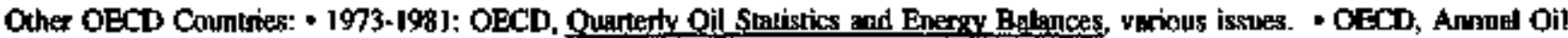
Statistics data base, 1970-1979. - 1982-1997: OECD, Monthly Oil Simtistics in the EIA Inemational Enera Dalabrase.

Oil Imports

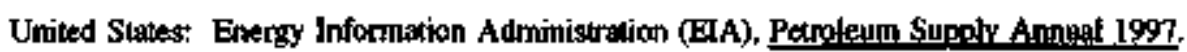

Other OECD Countries: - Organizalion for Economic Cooperalion and Development (OECD), Otarterly Oil Statistics and Enessy Balances, various issues. - OECD Monably Ot Statistics dalibase, 1991-1997. 
Appendlx A

Organizational and Geographical

Definitions 


\section{Appendix A. Organizational and Geographical Definitions}

Internotional Eoerny Agewcy (IFA) coumtries ane: Austrilia, Austria, Belgium, Canada, Denmark, Finland, France, Germany, Geeece, Ircland, ltaly, Japan, Luxembourg, Nethertands, Now Zealand, Norway, Portugal, Spxin, Sweden, Switzerland, Turkey, United Kingdoen, and Umiled States.

Organjantion for Economic Cooperatien and Development (OECD) countries are: Australia, Austria, Belgium, Canada, Czech Republic, Dermark. Finland, France, Germany, Greece, Hungary, keland, lretand, Italy, Japan, Luxembourg, Mexion, Netherlands, New Zealand, Norway, Poland, Portuggal,

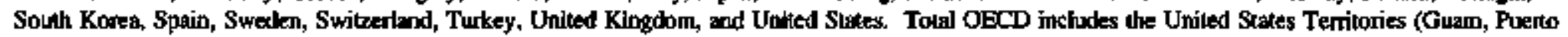
Rico, and the U.S. Virgin Islands). Talal OECD excludes data for Czech Republic, Hungary. Mexico, Poland, and South Korca which ate not yet available.

OECD Europe countries ace: Austria, Betgium, Czech Republic, Denmark, Finkand, France, Germany, Greece, Hungary, Icelend, Greland, Itoly, Luxembourg, Netherlands, Norway, Poland, Portugal, Spain, Sweden, Switzeriand, Turkey, and Uniked Kingdon. OECD Europe excludes data for Czech Republic, Humgary, and Potand which are not yet available.

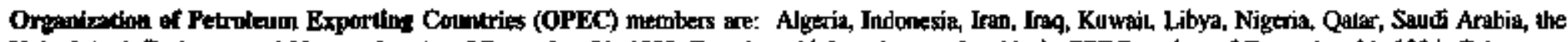
United Arab Emiratex, and Venezucla As of December 31, 1992, Ecuadkr withdrew its menbership in OPEC, and as of December 31, 1994, Gabon withdrew its membership. For consistency, Ecuador and Gabon are exchuded in the historical series of OPEC totals.

Arab menbers of OPEC are: Algeria, traç, Kuwaik, Libya, Qatar, Saudi Arabia and the United Arab Emirates.

Organization of Arab Petrolenn Exporting Countries (OAPEC) are: Algeria, Bahrain, Egypt, Iraq, Kuwail, Libya, Neutral Zone, Qatar, Sauci Arabia, Syria, Tunisia, and the United Arab Emirates. In April 1979 Egype was suspended fron OAPEC membership. In May 1989 Egypl rejoined OAPEC. For consistency. Egypt is inclided in the historical secies of the OAPEC totals.

Persian (Arabian) Gull countrles ane: Bahrain, Irat, braq, Kuwaith Qukar, Saudi Arobia, and the United Arab Emirates.

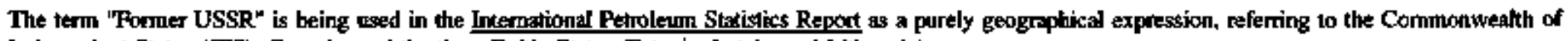
Independenl States (CIS), Georgia, and the three Ballic States (Estonia, Latvia, and Lithuania). 


\section{Appendix B}

\section{Explanatory Notes}




\section{Appendix B. Explanatory Notes}

\section{Noce I: Word Oil Supply}

Supply jnchudes production of crode oil (inchuding lease condensale), palural gas plank liguiks, other bydtogen and hydrocantons for refinery feedstocks, refinery gain, alcohol, and liquids produced from coal and other sources. Beginning in 1993, U.S. dnta includes fuel ethanol blendexd into finished motor gasofine and oxygenate prodpction frow merchank MTBE plants.

\section{Note 2: Oil Stocks}

The Internations Energy Agency (IEA) assembles and reports total oil stoct data for the menter countries of the Organization for Economic Cooperation and

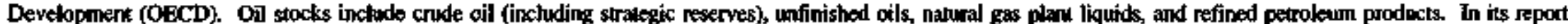
bitled Guantenty Oil Sanjstics ant Eneray Balances of CECD Comtries, the IEA defines oil stoxts as fotlows: "All non-military stocks beld by importers (inckuding all

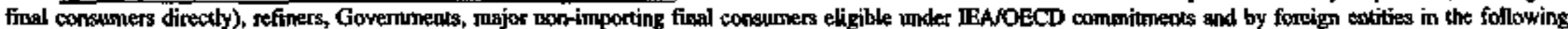
facilities: bull terminals, nefintery tanks, pipeline tankage, barges, intercoastal tankers (when pont of departure and destination ane in the reporting country), tankers in port (to the extent that their cargo is to be unboded in the reporting coustry), and inland ship bunkers. OAl is reported regardess of the ownersthip of such storage facilities." IFA data excluode "oil teld in pipelines, raij and trock tank cars, sea-going ships' burnkers, service stations, tetail stores and lankers at sea" Also the IEA defines the United States to be the 50 Stakes and the District of Columbia, and the U.S. TenTitocies (Gaam, Puerto Rtco, and the U.S. Virgin Islands). The Energy Information Adnínistretion (EIA) does not include the United Strtes Territories in its slecistical derintion of the United States. Further, the ElA includes oil in oil

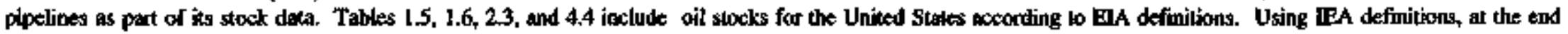
of Morch 1998 United States (including territories) oil stocks totaled 1,438 million barrels.

\section{Note 3: A Change to the Oil Balance in Section 2.}

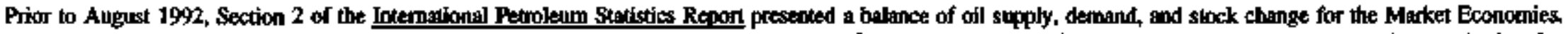

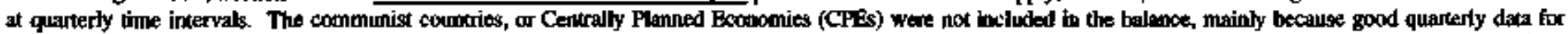
oil temand in these coentries were not available. Net imports from the CPEs were inchuded in the balaece as part of telal Matsel Bconomies suppty. At present, the former Sovict Union and nost of the countries of Eastern Burope ane in transition from commmism to matket economies, because of this there are no longer good

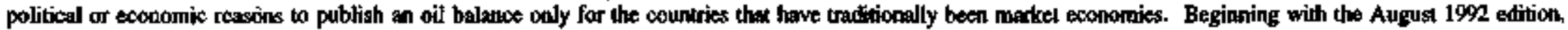

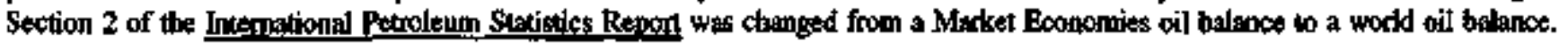

The main data issues related to this change may be sumnarized a follows:

Supply Dala. Montily cil supply dats are available for all countries of the wosld. These data are used to generate Tables 1.I to 1.4 ; they were used to expand the Scetion 2 supply table (Table 2.2) to the whole world.

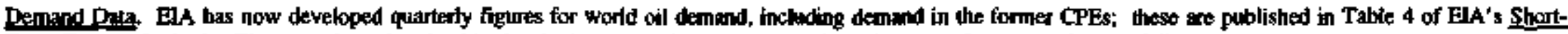
Term Energy Quelogk. The procedures involved in developing quartedy wortd demand nuntiers may be spmmanized as follows: 
Complete monthly data ane available for the 24 OECD countries. Annual demand data for all coantries (sbout 200 countries) are presented in EIA's International

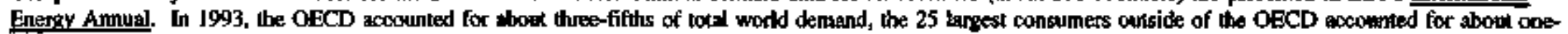
third of the workd lotil, and the remaining countries (about 150 countries) accounted for less then one-tenth of the totsil. The key problem, therefiore, is 10 develop good figures for the 25 largest consumers oulside the OECD.

Of these 25 countries, by far the largest consumer is Russia, which in 1993 accounud for atowt one-sixith of the demand in the group of 25 . The next largest consumer is China, which in 1993 accounced for abous ene-seventh of the demant in the group. Thus in 1993 Russia and Chima accoumed for ono-thind of the denarsd in the group of 25. For these 2 countries, data we available on oil production and net exports. The deonand numbers strown in Section 2 are compued as production mibus net exports. This is the same procedure used in the ElA Shout-Term Energy Outhook, ty the Intemational Energy Agency in their Monthly Oil Market Repors, and in

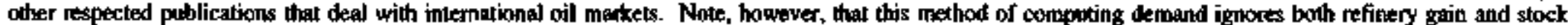
chenges.

For other majox consuming countries outside of the OECD, quirterly dement dace are developed based upon the latesl available annual dala, analytic judgment about concent trends, and some seasonal adjustmenls. These ane the same procodures used for the Short-Term Enerigy Outiock.

Stocks Data. Reliable stocks data are available for the OECD coumtries. Estinates of stocks for export and afloat, and of commercial floating stocks, ane available. (Ses sources at the end of Section 2.)

For on-land stocks oulside the OECD, there is bittle relistle data Most estimites of on-land stocks outside the OECD are based on the theory thet stocks are approximately equat to some number of days of temend. Demand figures (which themselves involve sone degree of estimation) ane then used to compate estinates of stock levels.

In the Intemational Petrotenm. Statistics. Repors it had been the practice for some time to estimate that on-land stocks in the traditional nerket economies outside of the OECD were equal to 55 drys of demand. This was acceptable, mainly because the rule was used only to estimate a relatively small part of total stocks (about 16 , percent).

When Section 2 was converted from a Market Economies gil balmce to a wodd oil baloce, there were importanx changes in the conditions that made it reasonable to extimale on-land stocts ontside of the OECD using the nule of 55 days of demand. When the CPEs are inchuded, the stocks thal must be estimated are a considerably larger part of the total. In 1990, total oil demind in the former CPEs was more then 80 percent as targe as demarad in all of the non-OECD traditixnal market ecooomics. Thus the quantity of stocks that would have to be estimsated using a 55 -day rule wonld nearly donble, and the estinated compontents of stock kevels and stock changes would become relatively large parts of the tobl. One conseqnence of this is thal the stotistical discrepancy (ns shown in Tabte 2.1) woukd become less useful as an indicator of the overall consisiency of the data. The statistical discrepancy is compulted from supply, demind, and stock change. If a large pan of the stock change is estimated, then the statistical discrepancy loses much of is significmpe.

As already noted, quarterly demands in the former Sowiet Unton and in China are computed as production mints net exports. This compotation implicitly assuntes a zero stock change. Demand in the former Soviot Union is falling. If wo were to ese a 55-day rule to conppute stock kevels, falling demand would imply falling stock levels. This would contratict the assumption of zero stock change which was used in compuling deminad. 
In view of all this, the following approach has been adopled. Table 2.3 now stows levels of stocks for the OECD conneries, levels of stocks for expont and afloat, and levels of commercial foaling storage. Stock changes based on these stock levels are shown im Table 2.l. At this time, no other stock levels are shown. Table 2.1 now has a single Iine labelled "Other Stock Draw and Stauistical Discrepancy", which contains the compated values needed to make the table balance.

It may become possible in the future to obtain reliable information en certain categories of on-land stocks outside the OECD. If such data become available, they will be added to Table 2.3, and the conresponding stock change information will be added to Table 2.1 .

Changes in Formats and Sign Conventions. There were several changes in Section 2 to make the Intesnational Petrokeum Statistics Report more consistent with other EIA publications, especially with the Short-Term Enefgy Q putlock. The mumbers were changed from 1 decimal place to 2 decimal places. The wond "consumption", as used before Augusi 1992, was changed to "demsand". The sign convention foe stodk changes in Table 2.1 was changet. Stock draws are now indicated hy posilive numbers, and stock additions are indicaled by tegative numbers. Table 2.1 also uses a new sign convention for the line labelkel "Oxher Stock Draw and Statistical Discrepancy". The values in this line are now computed as Tolal Demand minus the sutn of Tolal Supply and Total Reported Stock Draw. This is the reverse of the sigan convention used before August 1992.

Summary. Section 2 was expanded from a Market Economies oil balance to a warkd oil balance. Supply dafa ane tracked for all praducing countries. Demand daca are tracked for the 24 OECD countrtes, and for 25 large consutuers oulside of the OECD. Demand for the remaining countries (less than 10 percent of totil demand) is estimuiled, for years that are not yet available in the Intermational Enerxy Anonal. The stock levels in these tabies are based on reported dala for the OECD and for stocks at sea. The line in Table 2.1 labefled "Oher Stock Draw and Statistical Discrepancy" is a balancing itcm. 
Appendix C

Glossary 


\section{Appendix C. Glossary}

Alcebel. The family name of a goop of organic chemical compounds composed of carbesn, hydrogen, and oxygen. The malecules in the series vary in chain length and are composed of a hydrocarton phos a hydroxyl group. Alcohol includes methanol and ethasol.

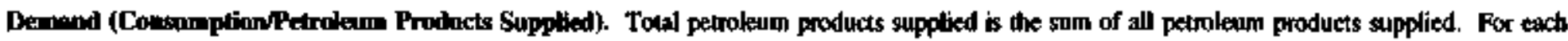
product, the amouni supplied is calculated by susnming production, crnde oil burned directly, imports, and net wilhdrawals from primary stocks and subtractituc exports.

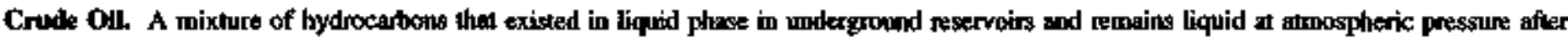
passing through surface-separating facilities. Cnude ofl production is measured at the welliced aod inchudes lesse condensate.

Govenment-0 wiwed Stacks. Oll stocks owned by the nationd govenment and held for nationsl security. Wh the United States these stocks are known as the Strategic Petroleum Reserve.

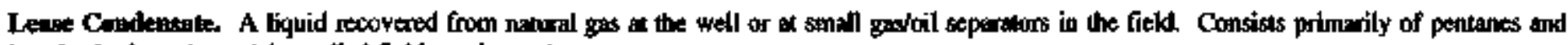
heavier hydtocarbons (also called field condensate).

Natural Gas Ptwot Lignids. Products obasined from processing natural gas a natural gas processing plants, including natural gasoline ptants, cycling plants, and fractionators. Products obtained include ethane, biquefied petroleum gases, (jroprimes, bulane, propante-botane mixtures, and

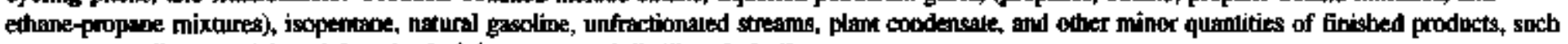
as mator pasoline, special naphthas, jet forth kerosene, and distilste fuel cil.

Oil Stecks. OAl stocks include conde oil (inctuding strutegic reserves), unfinished oilt, nalural gos piant liquids, and refined petroleum products. See Explanatory Note 2.

Other Hydrocmurbons. Other materials processed at refineries. Includes coal ir derivatives, hydrogen, gilsonite, and natural gas received by the refinery for reforming into hydrogen.

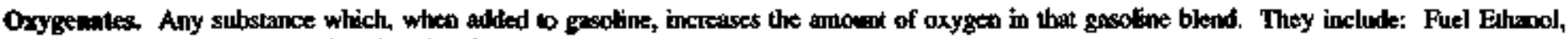
Methanot, and MTBE (Metryl tertiay butyl ctber).

Petrolening Products. Petrolenm products ane obtained from the processing of conde oil (inchuding lease condensate), natiural gas, anto odher

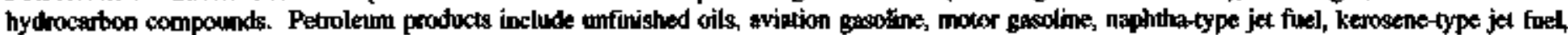
kerogene, distillate fuel oil, resitual fuel oil, ethane, liquefied petroleum gases, petrochemical feedstocks, special agphthas, lubricants, parafifie wax, petroleum coke, asphatt road oil, stil gas, and ather misctellaneous products.

Processing Gain. The anount by which the total welume of refincty ounpul is grester then the wolume of inpux for given period of time. The processing gain arises when crude oil snd other hydrocarbonts are processed imb products thet are, on average less dense thas the inpul.

Provesing Loss. The anount by which the tctal wilume of refinery output is less than the volume of inpun for given period of time. The

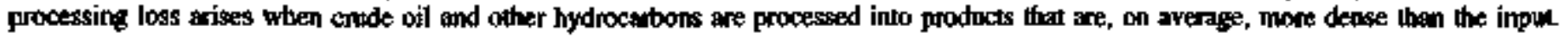


Appendix D

Estlmatlon Procedures 


\section{Appendix D. Estimation Procedures}

Estimation procedures are used for demard numbers in many of the countries outside of the CECD, in cases where data from EllA's International Energy Anmial ane not yot availoble. Demand numbers for 1996 and eatier years, for all countries, may be found in EIA's International Energy

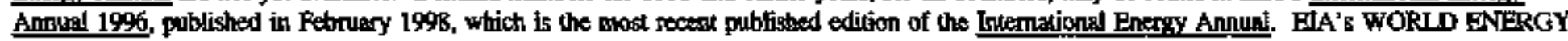
datasese contains finat demand data for all countries of the work for 1955, and preliminary demand data for all countries for 1996. Instructions for

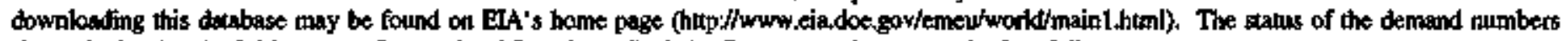

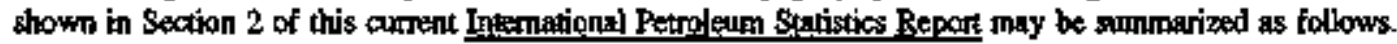

The OECD demand numbers are, as usual, based on the latest Queaterly Oil Stetisties and Monthly Oil Stetistics dala from the Internetional Encrog Agency.

For countries outside of the OBCD, the following anproach is used. The numbers for 1996 and earlies years mitch the dernand numbers in the WORLD ENERGY Jatabase on EIA's houne page. Numbers for 1997 forward ate the best aysilable BiA estimates, based upon the best anmaal data currently available, analytic judgmept about cument trends, and some seasonal adjustments. These are the sarne murnbers that appear in the international od bakance tabte in EIA's Short-Tern Enerxy Outkok.

The two largest wor-OECD oil consumers ane the former Soviel Union and Chint. For these two coumtries, publisthed dala are available on

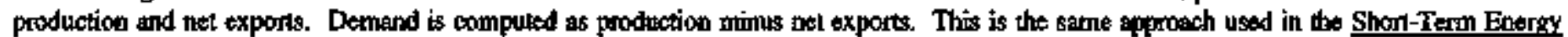
Ourtook, by the International Exergy Agency in their Monthly Oil Market Report and in olher major publictions dealing with international oil daca. Nole, however, that because of this compuration, the demand numbers for the former Soviet Union and China implicitly exclude stock draw and refinery gain. 
Appendix E

\section{Conversions}


Table E1. Refined Petroleum Products Conversion Factors

Prodoct

Barrels per Metric Tan

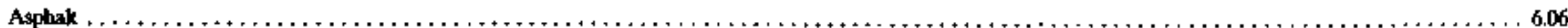

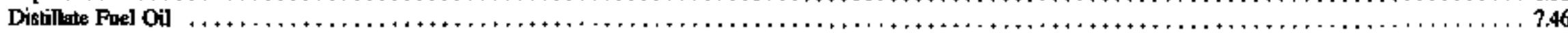

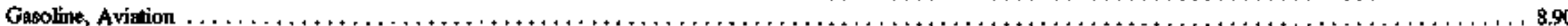

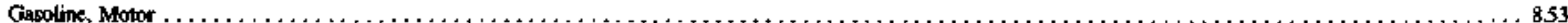

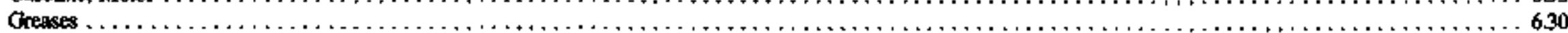

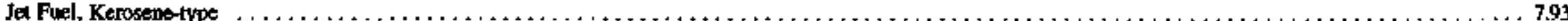

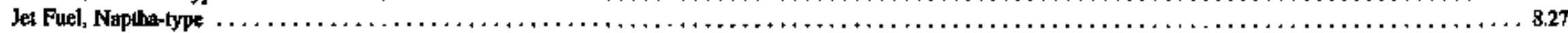

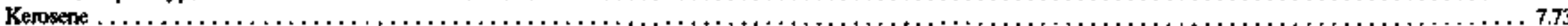

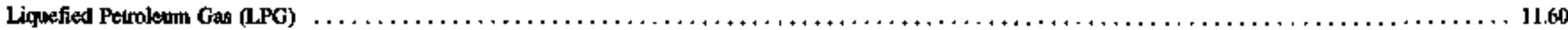

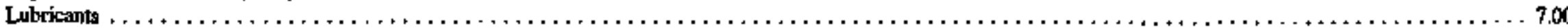

Mrscellaneous Produets

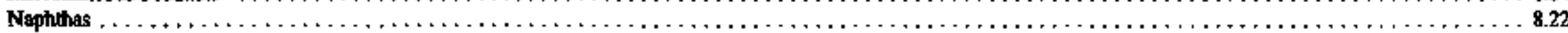

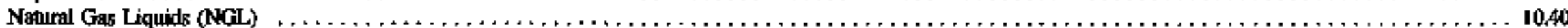

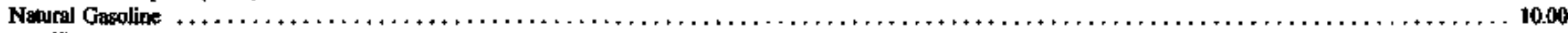

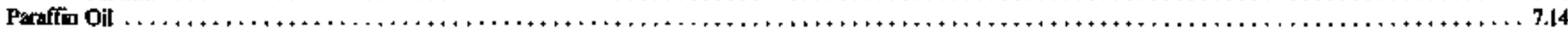

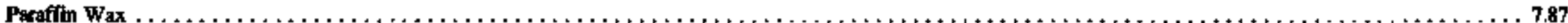

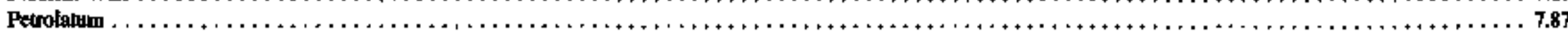

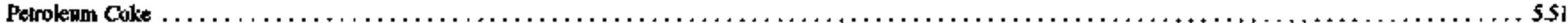

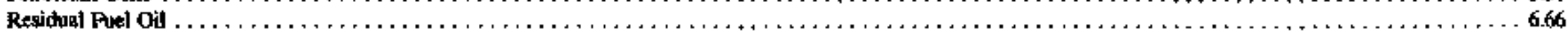

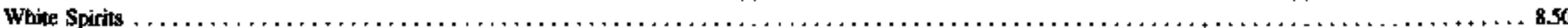


Table 12. Barrels of Crude od1 per watric ton, 1987-1996

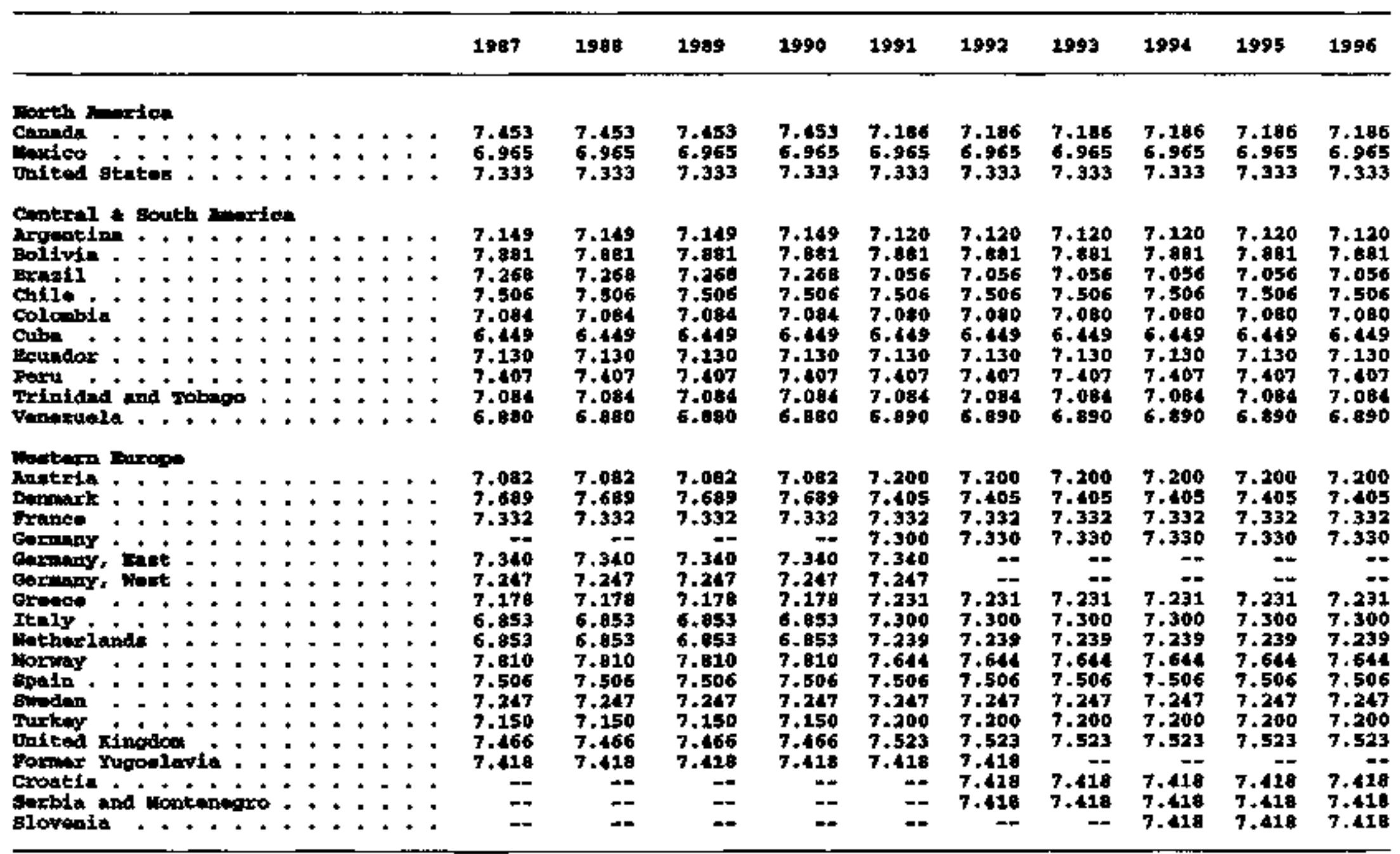

-- I W Protuction of thil tom of eargy.

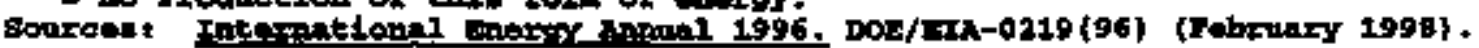


Table 72. Earme1s of Crude o12 por wotric Non, 1987-1996 (continued)

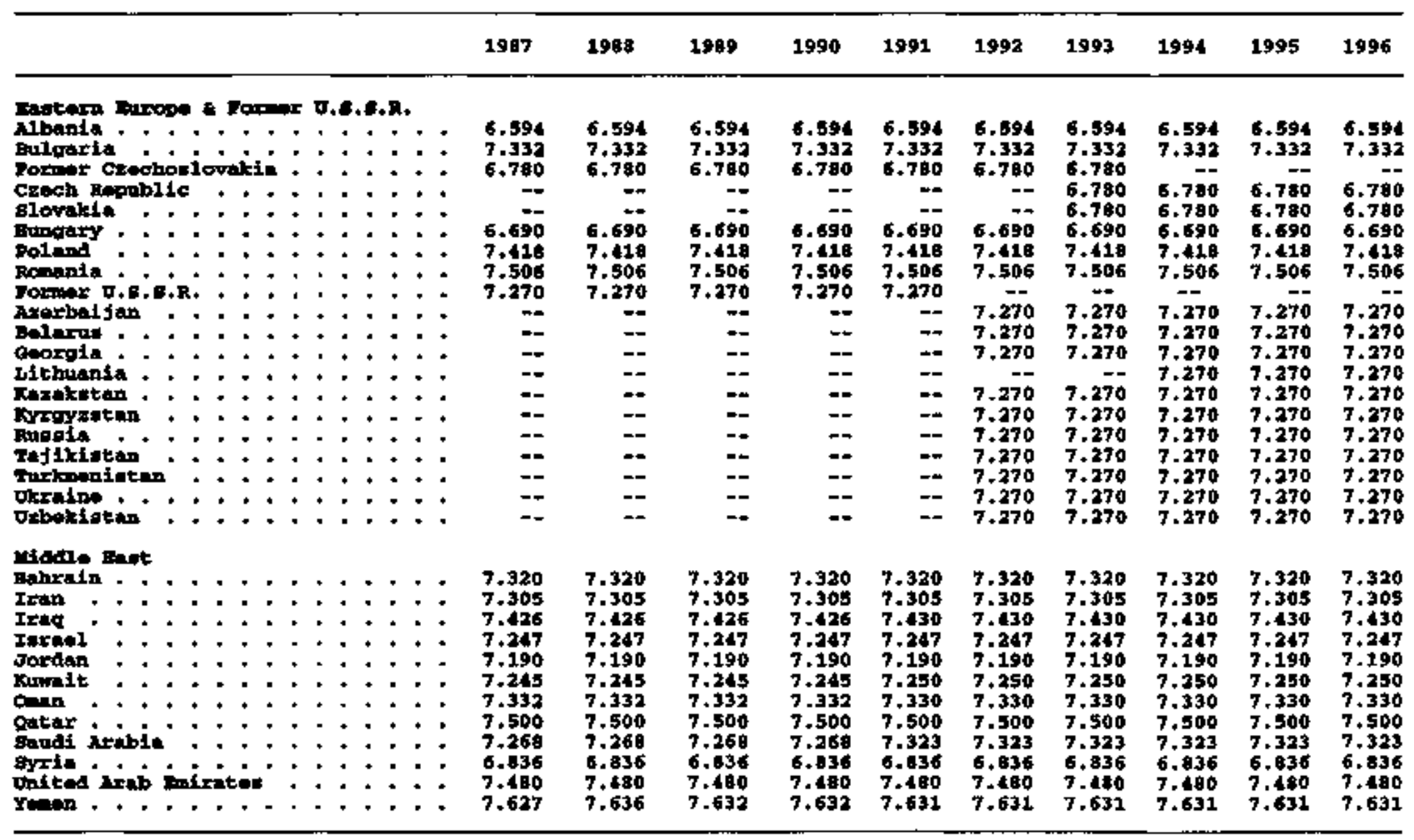

T- - Fo Production of this torn of energy.

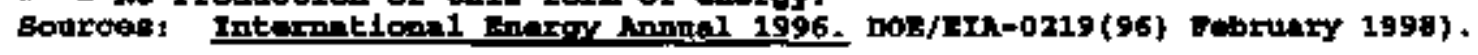


sble E2. Burrels of crude ofl per Jatrie Ton, $1987-1996$ (contimad)

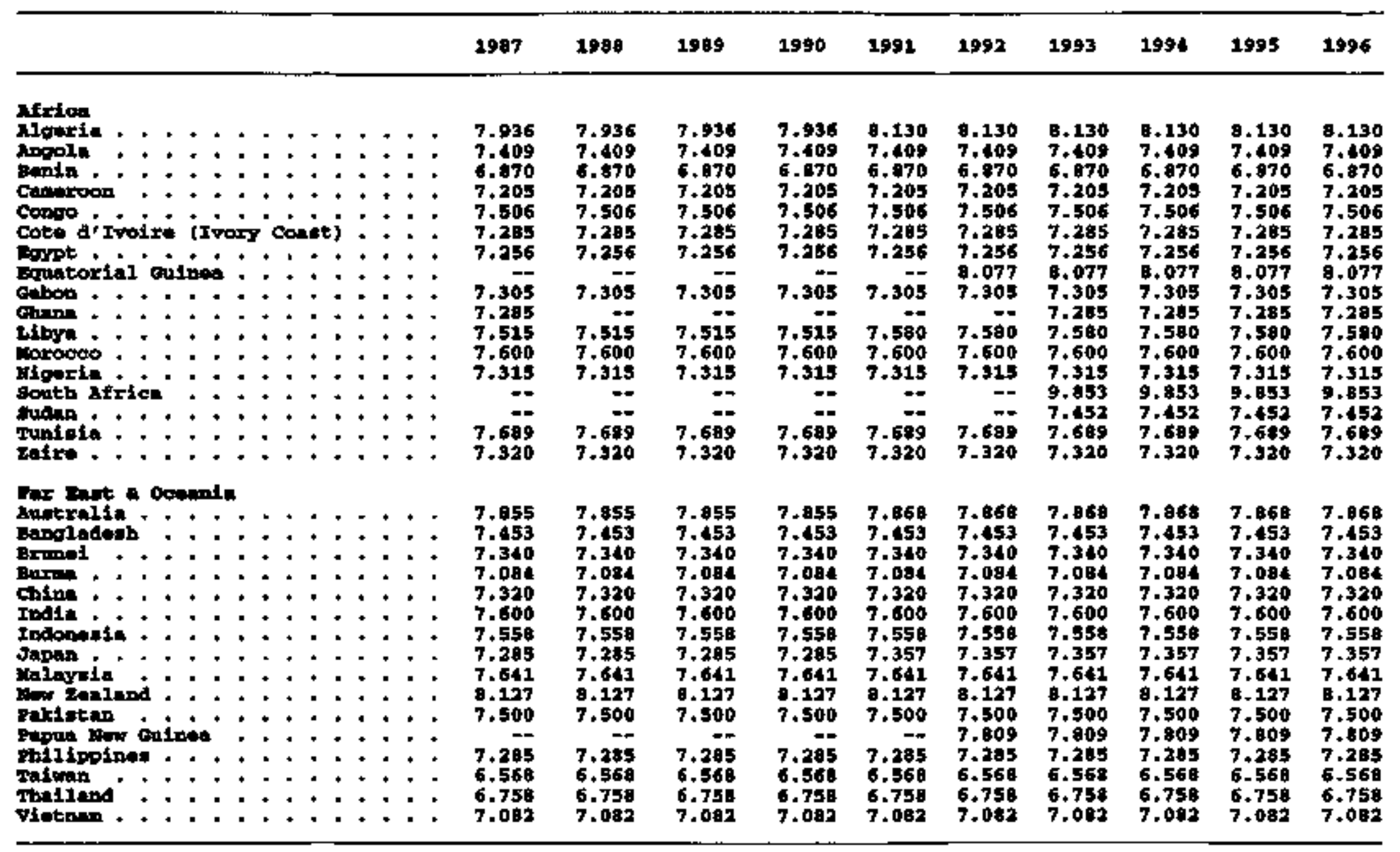

-- - wo Production of this form of sonery.

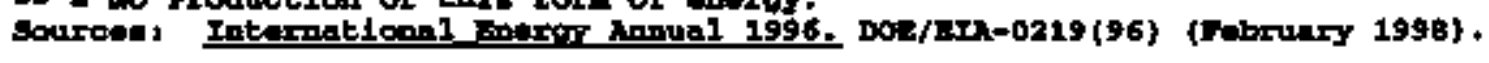

